\title{
A Comparison of Mortality Prediction Models for Neonatal Intensive Care Patients Using Artificial Neural Networks and Case-Based Reasoning
}

\author{
by \\ Saadia Khan, B. Eng.

\begin{abstract}
A thesis submitted to
The Faculty of Graduate Studies and Research in partial fulfillment of the requirements for the degree of Master of Applied Science in Electrical Engineering
\end{abstract}
Ottawa-Carleton Institute for Electrical and Computer Engineering Department of Systems and Computer Engineering Carleton University Ottawa, Ontario, Canada

September 2006

(C) 2006, Saadia Khan 


$\begin{array}{ll}\begin{array}{l}\text { Library and } \\ \text { Archives Canada }\end{array} & \begin{array}{l}\text { Bibliothèque et } \\ \text { Archives Canada }\end{array} \\ \begin{array}{l}\text { Published Heritage } \\ \text { Branch }\end{array} & \begin{array}{l}\text { Direction du } \\ \text { Patrimoine de l'édition }\end{array} \\ \begin{array}{l}\text { 395 Wellington Street } \\ \text { Ottawa ON K1A ON4 }\end{array} & \begin{array}{l}\text { 395, rue Wellington } \\ \text { Ottawa ON K1A ON4 } \\ \text { Canada }\end{array}\end{array}$

Your file Votre référence ISBN: 978-0-494-18319-9 Our file Notre référence ISBN: 978-0-494-18319-9

NOTICE:

The author has granted a nonexclusive license allowing Library and Archives Canada to reproduce, publish, archive, preserve, conserve, communicate to the public by telecommunication or on the Internet, loan, distribute and sell theses worldwide, for commercial or noncommercial purposes, in microform, paper, electronic and/or any other formats.

The author retains copyright ownership and moral rights in this thesis. Neither the thesis nor substantial extracts from it may be printed or otherwise reproduced without the author's permission.
AVIS:

L'auteur a accordé une licence non exclusive permettant à la Bibliothèque et Archives Canada de reproduire, publier, archiver, sauvegarder, conserver, transmettre au public par télécommunication ou par l'Internet, prêter, distribuer et vendre des thèses partout dans le monde, à des fins commerciales ou autres, sur support microforme, papier, électronique et/ou autres formats.

L'auteur conserve la propriété du droit d'auteur et des droits moraux qui protège cette thèse. $\mathrm{Ni}$ la thèse ni des extraits substantiels de celle-ci ne doivent être imprimés ou autrement reproduits sans son autorisation.
In compliance with the Canadian

Privacy Act some supporting forms may have been removed from this thesis.

While these forms may be included in the document page count, their removal does not represent any loss of content from the thesis.
Conformément à la loi canadienne sur la protection de la vie privée, quelques formulaires secondaires ont été enlevés de cette thèse.

Bien que ces formulaires aient inclus dans la pagination, il n'y aura aucun contenu manquant.

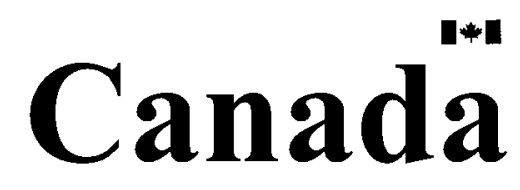




\section{Abstract}

This thesis attempts to find the minimum variable set that gives the best neonatal mortality prediction using day 3 data from the neonatal intensive care unit (NICU). A hybrid system is used consisting of an artificial neural network and the $\mathrm{kNN}$ case-based reasoner, which is verified as a valid imputation tool for missing values.

Two minimum variable sets were derived by reducing variables from linear and nonlinear network structures. The linear model obtained a higher average sensitivity and both day 3 models obtained higher areas under the ROC when compared with other models. 


\section{Acknowledgements}

First and foremost, I would like to thank my parents for encouraging me to finish and for being patient with my extended absences from home while I worked on this thesis. I would also like to thank my close friends and family who never failed to ask about my progress and consistently reminded me that I needed to finish.

I would like to thank Dr. Frize for her guidance and her constant optimism. I cannot imagine completing this thesis without her help. I am also grateful to the entire MIRG group, especially Dajie Zhou and Dr.Walker, for their help and support.

I would also like to give a special thanks to my section head, trainer, and division head who were understanding of my commitments outside of work and allowed me time off work to complete this thesis. 
The undersigned recommends to

the Faculty of Graduate Studies and Research

acceptance of the thesis

\author{
A Comparison of Neonatal Mortality Prediction Models using \\ Artificial Neural Networks and Case-Based Reasoning \\ submitted by \\ Saadia Khan, B.Eng \\ in partial fulfillment \\ of the requirement for the degree of \\ Master of Applied Science in Electrical Engineering
}

Thesis Supervisor

Chairperson, Department of Systems and Computer Engineering

Carleton University

September 2006 


\section{Contents}

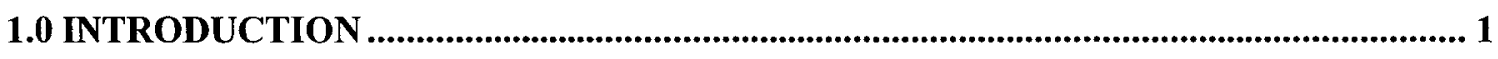

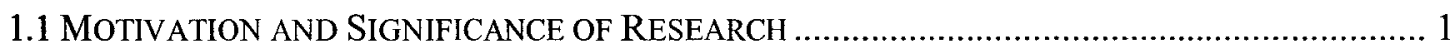

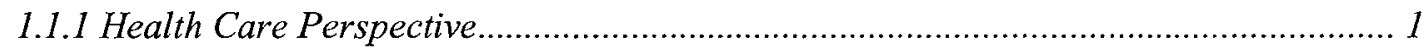

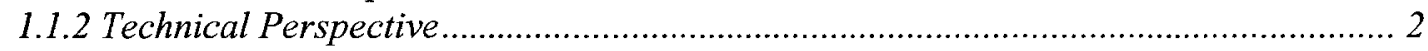

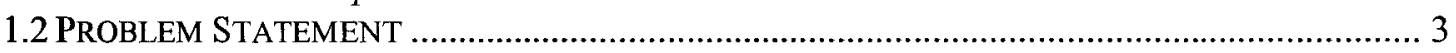

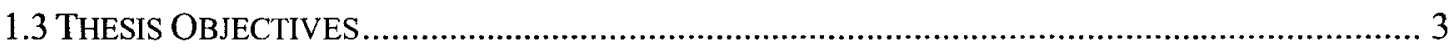

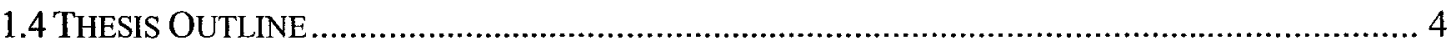

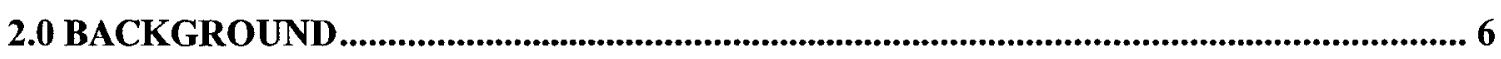

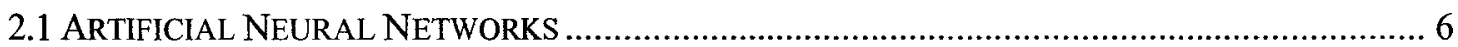

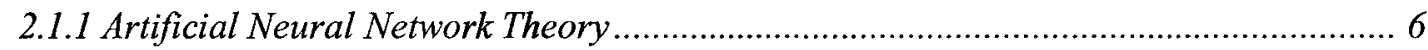

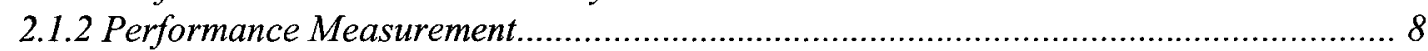

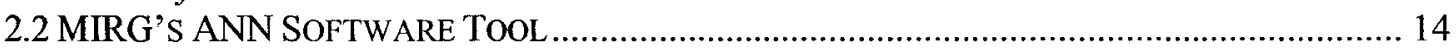

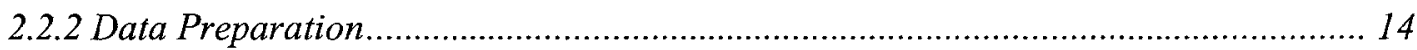

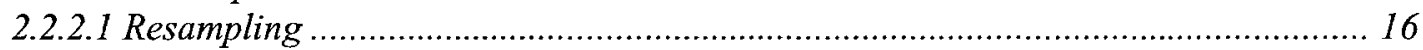

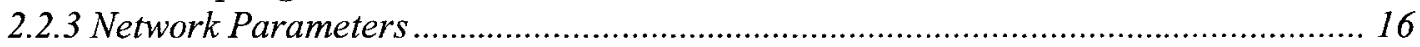

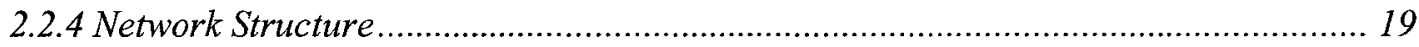

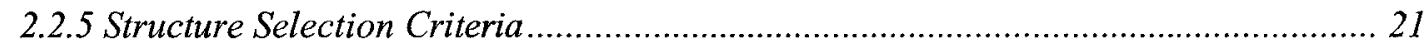

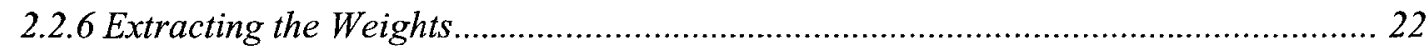

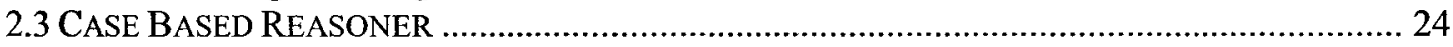

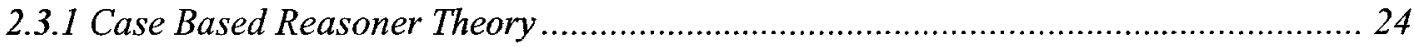

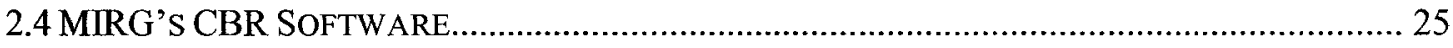

2.4.1 The Need for Imputation of Missing Values in NICU Data ..................................... 26

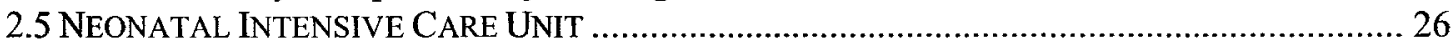

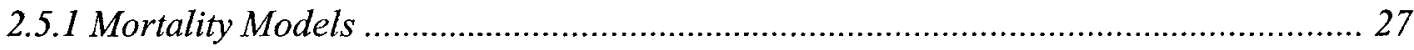

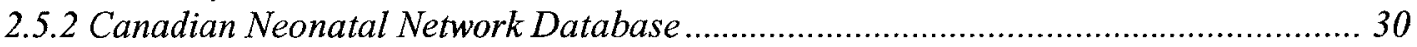

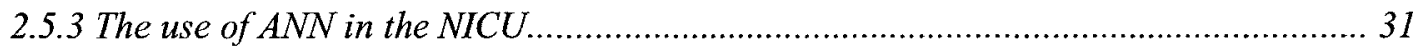

3.0 LITERATURE REVIEW AND RELEVANT WORK ................................................... 34

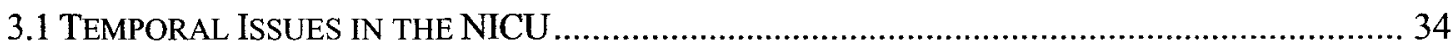

3.1.1 Issues to Consider for Temporal Clinical Decision Support Systems ....................... 34

3.1.2 Trends in Time of Death in the NICU................................................................ 35

3.1.3 Temporal Effects on Physician Prediction and Confidence ...................................... 37

3.1.4 The Relationship between Time and Contamination-free Prediction......................... 38

3.1.5 Temporal Effects on Resource Allocation ............................................................ 40

3.2 MEDICAL IDEAS RESEARCH GROUP ......................................................................... 41

3.2.1 Research in Predictive Power of Day 3 Data .......................................................... 41

3.2.2 Developing a Neonatal Mortality Prediction Model using an ANN-CBR Hybrid....... 43

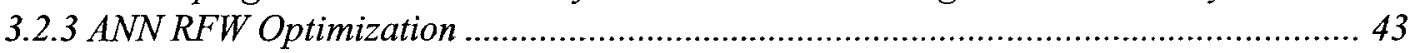

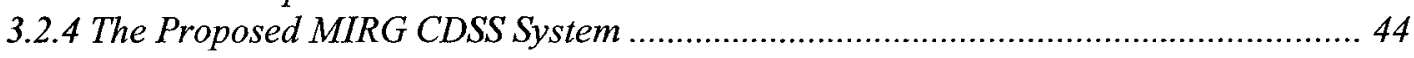

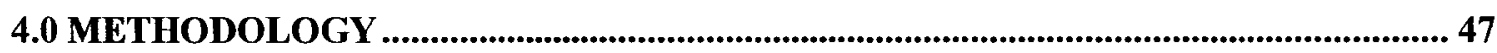

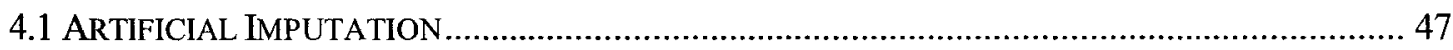

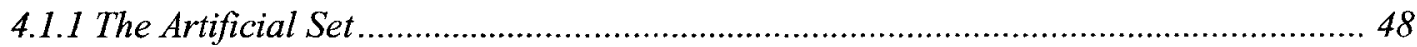

4.1.1.2 Artificial Imputation Using Linear and Non-linear Weights............................... 50

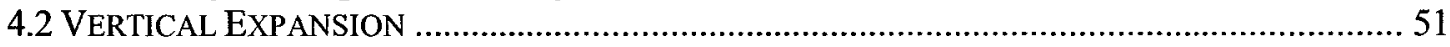

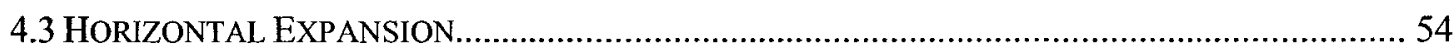




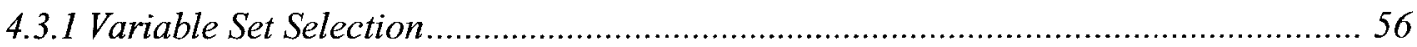

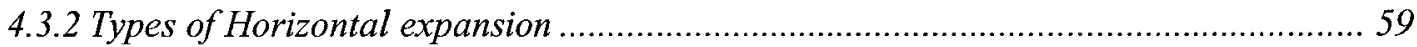

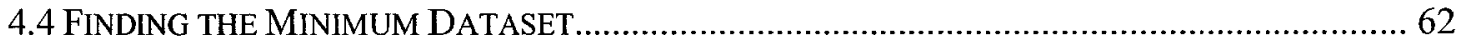

4.4.1 Variable Reduction using Linear Network and Non-linear Network Structures ......... 62

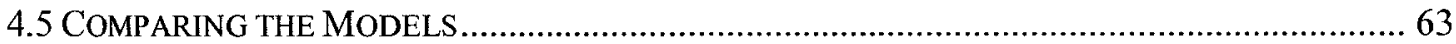

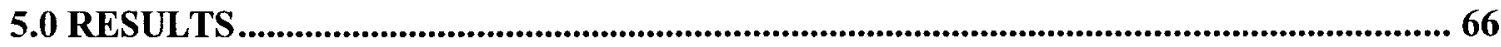

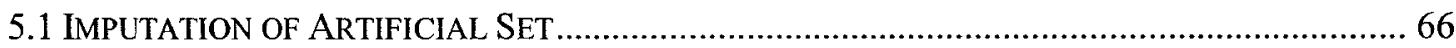

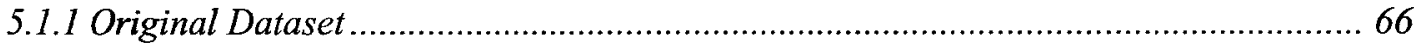

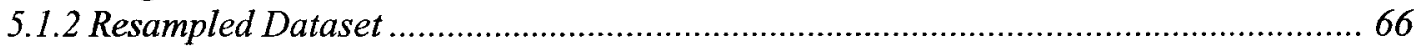

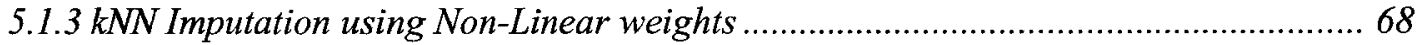

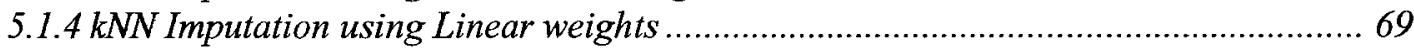

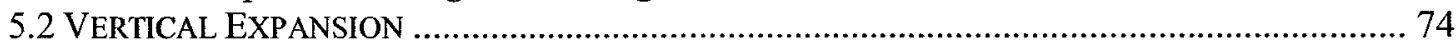

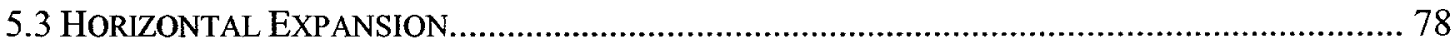

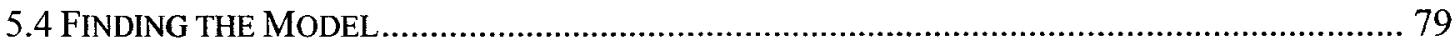

5.4.1 Using linear structure to find Minimum Data Set ................................................... 79

5.4.2 Using a Non-linear Structure to find Minimum Data Set........................................ 83

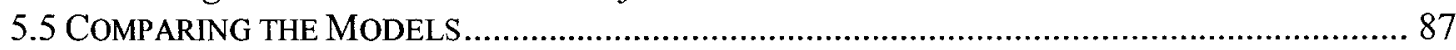

5.5.1 Analysis of the Best Performing Structure ....................................................... 90

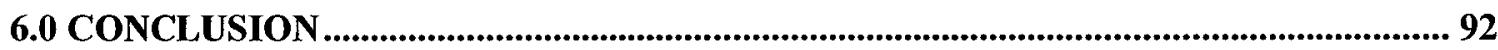

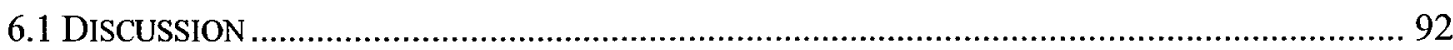

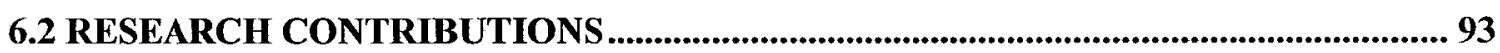

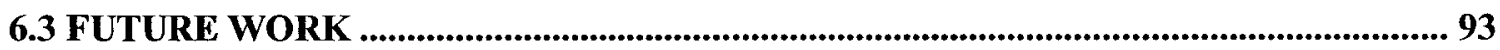

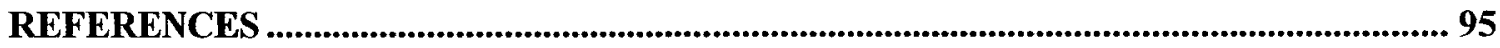

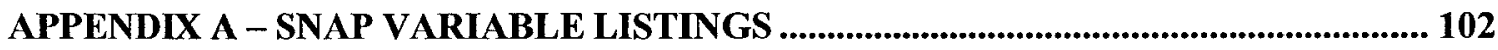

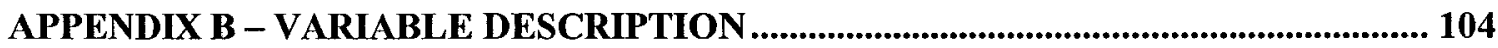

APPENDIX C - VARIABLE REDUCTION RESULTS ...................................................... 106

APPENDIX D - BEST PERFORMING STRUCTURE RESULTS ..................................... 114

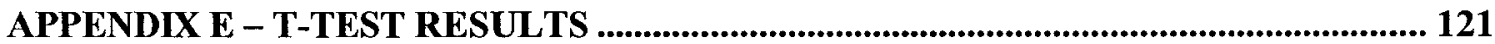




\section{List of Figures}

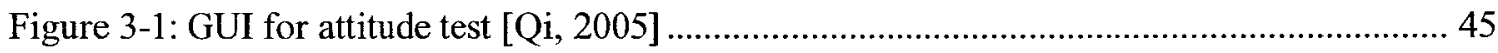

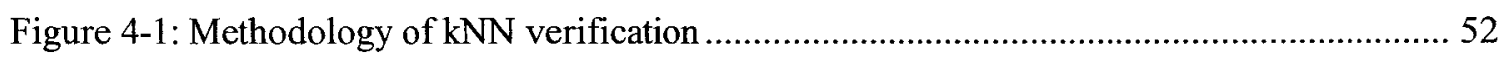

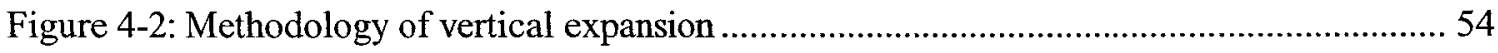

Figure 4-3: Step-wise horizontal imputation of day 3 database for 28 variables $(\mathrm{N}=2364) \ldots \ldots . . .60$

Figure 4-4: Methodology of horizontal expansion, variable reduction and model comparison.... 63

Figure D-1: Three-layered network with two hidden nodes and SNAPPE-II variable set using day

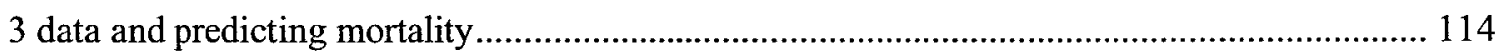

Figure D-2: Two-layered network (0 hidden nodes) result for Ennett's model dataset using day 3

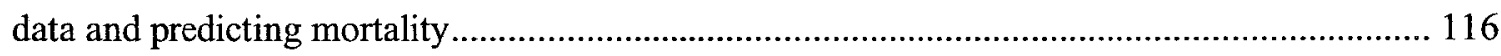

Figure D-3: Three-layered structure with four hidden nodes using 13 variable linear model and

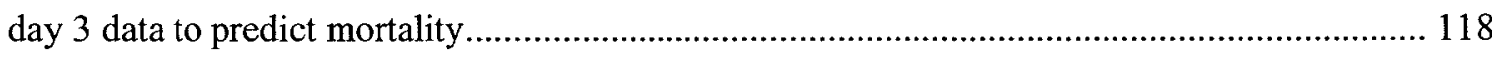

Figure D-4: Three-layered structure with four hidden nodes using 13 variable non-linear model and day 3 data to predict mortality 


\section{List of Tables}

Table 2-1: A visual representation of the relationship between actual and prediction outcomes and true and false predictions [Hohavi and Provost, 1998]

Table 4-1: Missing value statistics for each of the SNAPPE-II variables in the day 3 database (N

$=14,261)$ and artificial dataset $(\mathrm{N}=2,364)$

Table 4-2: Missing SNAPPE-II value statistics for entire day 3 database $(\mathrm{N}=13,871)$ based on number of missing values

Table 4-3: Training, test and verification set statistics for complete cases in day 3 database $(\mathrm{N}=$ 2,364)

Table 4-4: Training, Test and verification set statistics for entire day 3 database after vertical imputation $(\mathrm{N}=13,871)$. 54

Table 4-5: Missing value statistics for all 40 variables in day 3 database $(\mathrm{N}=14,261) \ldots \ldots \ldots \ldots . . .56$

Table 4-6: Training, test and verification sets for set $A$ of the horizontal imputation $(\mathrm{N}=1,700) 58$

Table 4-7: Training, test and verification sets for set $\mathrm{B}$ of the horizontal imputation $(\mathrm{N}=1,077) 58$

Table 4-8: Training, test and verification sets for set $\mathrm{C}$ of the horizontal imputation $(\mathrm{N}=552)$.. 59

Table 4-9: Missing value statistics for entire day 3 database by number of missing values $(\mathrm{N}=$ $13,871)$.

Table 4-10: Missing value statistics for cases missing values for the set $A$ variables $(N=2,364) 61$

Table 4-11: Missing value statistics for cases missing values for set $\mathrm{B}$ variables $(\mathrm{N}=2,364) \ldots . .61$

Table 4-12: Missing value statistics for cases missing values for set $\mathrm{C}$ variables $(\mathrm{N}=2,364) \ldots . .61$

Table 4-13: Training, test and verification sets for horizontally imputed database $(\mathrm{N}=2,364) \ldots 62$

Table 4-14: Training, test and verification sets for imputed database for 13 variable linear model

$(\mathrm{N}=13,871)$. 64

Table 4-15: Training, test and verification sets for imputed database for 13 variable non-linear model $(\mathrm{N}=13,871)$ 64

Table 4-16: Training, test and verification sets for imputed database for 13 variable Ennett model $(\mathrm{N}=13,871)$ 64

Table 5-1: ANN Performance of original day 3 dataset $(\mathrm{N}=2,364)$ for predicting mortality. 66

Table 5-2: ANN Performance results of resampled day 3 dataset for predicting mortality $(\mathrm{N}=$ 2,364) 67

Table 5-3: Variable weights extracted from the non-linear network with four hidden nodes (wnot $=0.4996)$ 68 
Table 5-4: A comparison of the P values from day 3 imputation using the kNN compared to day 1 imputation using the CBR 68

Table 5-5: ANN performance results for artificial set imputed using non-linear weights $(N=2,364)$

Table 5-6: ANN performance results for imputed database using linear weights $(\mathrm{N}=2,364) \ldots \ldots .70$

Table 5-7: Variable weights from two-layered network ................................................................. 70

Table 5-8: SNAPPE-II variable order of importance (starting with most important) ................... 71

Table 5-9: T test (sig-2) results for imputed datasets using linear and non-linear weights ........... 72

Table 5-10: Mean and standard deviation comparison of original dataset with imputed datasets 72

Table 5-11: ANN performance results for imputed dataset using linear weights $(\mathrm{N}=13,871)$...... 73

Table 5-12: Ennett's ANN performance results for day 1 data using the best test set performance $(\mathrm{N}=5,102)$.

Table 5-13: Mean and standard deviation changes in the complete dataset as cases missing an increasing number of missing values are added

Table 5-14: ANN performance results for vertically imputed database of SNAPPE-II variables (N

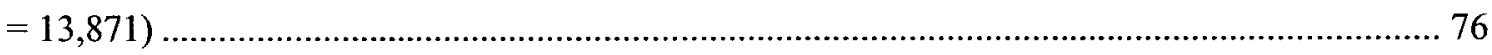

Table 5-15: Ennett's results for 13 input variables [Ennett, 2003] ................................................ 76

Table 5-16: ANN performance results for 13 variable Ennett model on day 3 data $(\mathrm{N}=13,871)$. 77

Table 5-17: Variable weights used in horizontal imputation 79

Table 5-18: ANN performance results on two-layered network as variables are gradually removed $(\mathrm{N}=2,364)$ 80

Table 5-19: Variable weights on linear network as least important variables are gradually removed

Table 5-20: A comparison of the 10 variable day 3 linear model, the 13 variable day 1 model found by Ennett and the SNAPPE-II model. 83

Table 5-21: ANN performance results using non-linear network as variables are gradually removed $(\mathrm{N}=2,364)$

Table 5-23: A comparison of the 10 variable model found on linear network compared with the 9 variable model found on a non-linear network for day 3, Ennett's day 1 model and the SNAPPE-II model.

Table 5-24: ANN performance results for linear 13 variable model for full day 3 database $(\mathrm{N}=$ $13,871)$ 87

Table 5-25: ANN performance results for 13 variable non-linear model for full day 3 database $(\mathrm{N}$ $=13,871)$ 88 
Table 5-26: A comparison of the best performing ANN structure for the four different mortality

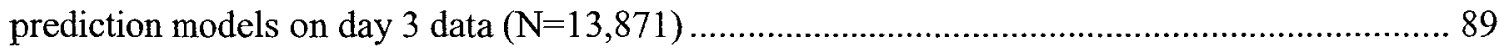

Table A-1. SNAP variable list and scoring system [Richardson et al., 1993] ........................... 102

Table A-2. SNAP-II variable list and scoring system [Richardson et al. 2001] .......................... 103

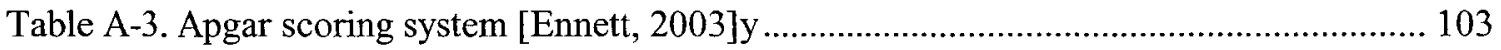

Table B-1: SPSS variable name and transformation formulae for SNAP variables [Ennett, 2003]

Table C-1: Initial parameters of ANN used in model reduction of linear and non-linear network structures 106

Table C-2: ANN RFW parameters of best test run for linear tests during variable reduction step 106

Table C-3: Complete training, test, and verification set results of 28 variable linear test........... 107

Table C-4: Complete training, test and verification set results of 22 variable linear test............ 107

Table C-5: Complete training, test and verification set results of 16 variable linear test............ 108

Table C-6: Complete training, test and verification set results for 15 variable linear test .......... 108

Table C-7: Complete training, test and verification set results for 14 variable linear test .......... 109

Table C-8: Complete training, test and verification set results for 13 variable linear test .......... 109

Table C-9: ANN RFW parameters of best test run for non-linear tests during variable reduction step

Table C-10: Complete training, test, and verification set results of 28 variable non-linear test . 110 Table C-11: Complete training, test and verification set results of 22 variable non-linear test .. 111 Table C-12: Complete training, test and verification set results of 16 variable non-linear test .. 111 Table C-13: Complete training, test and verification set results for 15 variable non-linear test 112 Table C-14: Complete training, test and verification set results for 14 variable non-linear test 112 Table C 15: Complete training, test and verification set results for 13 variable non-linear test . 113 Table D-1: Initial ANN RFW parameter values for SNAPPE-II set and Ennett's model set (tables 31 and 33) 114

Table D-2: Complete training, test and verification set results for two hidden nodes and SNAPPEII variable set using day 3 data and predicting mortality 115 Table D-3: Complete training, test and verification set results for zero hidden nodes and Ennett variable set using day 3 data and predicting mortality 116

Table D-4: Initial ANN RFW parameter values for 13 variable linear model for day 3 data .... 117 Table D-5: Complete training, test and verification set results for four hidden nodes and 13 variable linear model using day 3 data and predicting mortality 
Table D-6: Initial ANN RFW parameter values for 13 variable non-linear model for day 3 data

Table D-7: Complete training, test and verification set results for four hidden nodes and 13 variable linear model using day 3 data and predicting mortality 120

Table D-8: ANN RFW parameters of best test run for best performing structure for each model 


\section{List of Equations}

Equation 2-1: Classification Rate [Hohavi and Provost, 1998] ............................................... 9

Equation 2-2: Average Squared Error [Haykin, 1999; pg. 172] .......................................... 10

Equation 2-3: True negative and false negative calculations [Hohavi and Provost, 1998] .......... 11

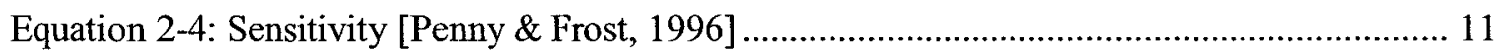

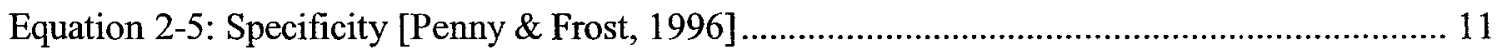

Equation 2-6: Log sensitivity [Ennett et al., 2002; Scales, 2001] .......................................... 12

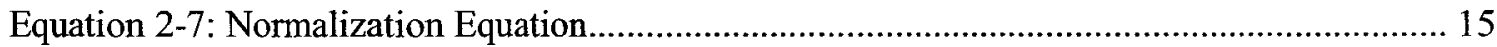

Equation 2-8: Adaptive ANN changes using the error ratio [Ennett, 2003] ............................... 18

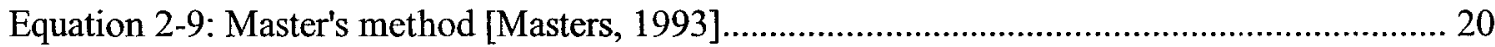

Equation 2-10: Livingstone and Manallack's method [Livingstone and Manallack, 1993] .......... 20 


\section{Glossary}

Apgar score: An additive scoring system usually taken 1, 5 and 10 minutes after birth to assess risk of complication or bad outcome. APGAR = Appearance, Pulse, Grimace, Activity, Respiration. Score is out of 10 points.

Artificial neural network: An artificial intelligence tool that identifies patterns in databases to predict outcomes. Two-layer networks (i.e. input and output layers) are linear. Networks with three or more layers (i.e. one or more hidden layers) are nonlinear.

Case-based reasoner: An artificial intelligence tool that uses past case histories to predict future outcomes and match similar cases. Particularly useful when working with text fields in databases. Most commonly used for retrieval of similar cases to the query. Complicated and knowledge-intensive updating procedures make it difficult to completely automate the system.

MIRG: Medical Information technologies Research Group located in Ottawa, ON. Principle investigators: Dr. Monique Frize, P.Eng., O.C. (Carleton University and University of Ottawa) and Dr. C. Robin Walker, MBBS, FRCPC (Halifax).

Nearest neighbours algorithm: An algorithm that calculates the distance between two points in an n-dimensional space. The k-nearest neighbours algorithm finds the k closestmatching cases to the query. A weighted-nearest neighbours algorithm allows the system parameters to be weighted differently as opposed to uniform weighting. Generally used for pattern classification.

Neonatal death: Defined by the World Health Organization as death occurring before 28 days of age.

SNAP: Score for Acute Neonatal Physiology; contains 37 input variables and is validated for predicting mortality and is highly correlated with nursing workload, length of hospital 
stay and therapeutic intensity in the Neonatal Intensive Care Unit (NICU).

SNAPPE: SNAP with Perinatal Extension; uses the 37 SNAP variables plus birth weight, small for gestational age and Apgar score at 5 minutes.

SNAP-II: SNAP Version 2; a parsimonious version of SNAP with only six input variables (of the original 37 inputs) and is validated for predicting mortality, severe intraventricular hemorrhage and chronic lung disease in the NICU.

SNAPPE-II: SNAP-II with Perinatal Extension; uses the six SNAP-II variables plus birth weight, small for gestational age and Apgar score at 5 minutes. 


\section{Nomenclature}

ANN: Artificial neural network

APACHE: Acute physiology and chronic health evaluation

CBR: Case-based reasoner/reasoning

CRIB: Clinical risk index for babies

ICU: Intensive care unit

NICU: Neonatal intensive care unit

NTISS: Neonatal therapeutic intervention scoring system

ROC: Receiver operating characteristic curve

SGA: Small for gestational age $(<5$ th percentile)

SNAP: Score for neonatal acute physiology

SSE: Sum of squared errors 


\subsection{Introduction}

\subsection{Motivation and Significance of Research}

Although in the $21^{\text {st }}$ century most premature and unhealthy newborns ultimately survive, there still exists a small number of infants for whom difficult treatment decisions must be made. With the aid of artificial intelligence tools clinicians and parents are more informed of the possible outcomes and the optimal course of treatment for their neonate. The outcome prediction has been improving in the past few years given only data collected upon admission to the intensive care unit [Richardson et al., 1998; Meadow et al., 2004]. However, it is important to establish whether more recent data would help improve the prediction accuracy. This research is motivated by a need for accurate outcome prediction using data collected after the admission day.

\subsubsection{Health Care Perspective}

In recent years the likelihood of survival for neonates has improved significantly due to better obstetric care before the infant is admitted into the neonatal intensive care unit (NICU) and better NICU care after admission [Richardson et al., 1998]. Of the infants that do not survive, most perish within the first day in the NICU. Predicting who will ultimately survive of the remaining infants is the lingering question.

The mean length of survival has also increased in parallel with the improved survival rates [Meadow et al., 2004]. This suggests that it takes longer to assess the status of the very ill infant and in the meantime, the infant consumes clinician time and NICU resources. Conventional mortality prediction is calculated using physiological parameters collected on the first day or admission day in the NICU. In the present day, infants that will eventually die live longer than before and because of this, the admission day data loses much of its predictive power. It has been observed that some infants, especially those of very low birth weight, ultimately die because of events or conditions that are not present upon their admission into the NICU, but arise later in their stay [Richardson et al., 2001]. It has also been observed that physicians' predictions cannot be solely relied upon for important ethical decisions as there is an overload of 
information that cannot be easily integrated, accessed and assessed to aid the physician in decision making.

The new survival trends in the NICU have given rise to a number of new issues. To address these new issues a new prediction methodology must be considered. This research looks at the predicting power of data collected on day 3 of NICU with the expectation that, as time progresses and as infants near their ultimate outcome, the data collected at day 3 may provide a more accurate prediction of the eventual outcome. For example, conditions and events, such as an infection that cannot be seen upon admission, may be better represented by day 3 data.

This research is also motivated by the need for longitudinal monitoring of physiological data in the NICU as has been suggested in many studies [Sankaran et al., 2002]. Real-time monitoring has many advantages such as the ability to monitor trends in an individual's state. Similarly, real-time outcome prediction has the ability to track and utilize trend information not considered by admission day prediction.

\subsubsection{Technical Perspective}

The Medical Information technologies Research Group (MIRG) has been developing artificial neural network (ANN) tools to predict outcomes such as mortality, ventilation length, length of stay, and the occurrence of complications in the clinical environment, based on certain physiological parameters. In addition, MIRG has been developing a case based reasoner (CBR) tool that is useful in matching patient cases that have similar input parameters, and therefore, potentially similar outcomes. The ANN tools are in the pilot testing stage as they have been integrated with user interfaces for testing purposes [Qi, 2005]. An ethical decision aid has also been piloted, that uses the ANN outcome results to aid parents and guardians participating in the decision on whether or not to withdraw their child from intensive care treatment [Yang, 2004; Frize et al., 2005].

The ultimate goal of MIRG is to develop an on-line outcome prediction system that receives real-time data input and computes outcomes in real-time for a remote user. The system will be designed for possible integration with a hospital information system, 
such as the data repository at the Children's Hospital of Eastern Ontario (CHEO), which presently captures and stores physiological data for each infant in real-time.

An essential first step in the construction of a real-time prediction system, is the study of the temporal effects on ANN prediction power. This is the main focus of this research. The research compares the predictive power of data collected on the third day of NICU stay with the predictive power of admission day data. In addition, the development of a new mortality prediction model specially adapted to use day 3 data, is explored.

\subsection{Problem Statement}

There has been much research in the area of neonatal mortality prediction and clinician prediction. Also, the study of neonatal survival trends and resource allocation as time passes in the NICU have been popular research topics recently. Although, both mortality prediction and temporal effects in the NICU have been separately studied, the effects of time on mortality prediction have not been addressed. Thus far, mortality prediction models specially adapted to predict mortality on days after NICU admission have not been developed. This research aims to address this gap in knowledge.

By attempting to discover a mortality prediction model for day 3 of an infant's NICU stay and comparing its performance with admission day prediction it is hoped that the findings of this research will be an important building block in the development of an on-line, real-time prediction system for the NICU.

\subsection{Thesis Objectives}

The goal of this research is to develop a neonatal mortality model using day 3 data with the assumption that the new model will improve the prediction performance. This objective is pursued through the following six steps:

1) The ability of the updated case based reasoner tool $(\mathrm{kNN})$ to impute missing values into a dataset is verified by the imputation of known data values

2) Missing value imputation using weights extracted from a linear (2-layered) neural network is compared to imputation using weights extracted from a non-linear (3layered) neural network 
3) The kNN is then used to impute missing values to "vertically" expand the day 3 dataset, so a greater number of cases are used to train the ANN [Ennet, 2003]

4) The kNN is used to impute missing variables to "horizontally" expand the day 3 dataset, so a greater number of variables are considered in the creation of the mortality prediction model for day 3 data [Ennett, 2003]

5) Compare the minimum data set found using a linear network with the minimum data set found using a non-linear network

6) Find the 3-layered neural network structure of the $2 n+1$ structures (where $n$ is the number of input variables to the network) that gives the best performance on day 3 data

The approach taken in finding the day 3 mortality model is similar to the approach taken by Ennett of MIRG to develop a mortality model for day 1. In this research a CBR tool $(\mathrm{kNN})$ developed by MIRG is verified as a valid imputation tool in place of the commercial tool used by Ennett. This research also compares the performance of a linear network structure with non-linear structures to determine which gives better weights for missing value imputation and which, gives a better performing minimum data set.

\subsection{Thesis Outline}

Chapter 2 explains the theory behind artificial neural networks and case based reasoners and their application in the clinical environment. It also covers the neonatal intensive care environment and some neonatal mortality prediction models.

Chapter 3 discusses the issues in the NICU that pertain specifically to the effects of time on the prediction of mortality. The chapter also gives a brief overview of relevant MIRG research in the field.

Chapter 4 elaborates on the problem statement and methodology used in the research. It explains in detail, the initial $\mathrm{kNN}$ verification step and the two-step imputation process that was performed in order to derive a mortality model for day 3 .

Chapter 5 discusses the results of the $\mathrm{kNN}$ verification step and the imputation steps. It also looks at the day 3 linear and non-linear models derived from the ANN tool and compares their performance with the MIRG mortality model used in the past. 
Chapter 6 concludes with a discussion of the results, the contributions of this research and future work that is required to progressively develop the final real-time mortality prediction system. 


\subsection{Background}

\subsection{Artificial Neural Networks}

\subsubsection{Artificial Neural Network Theory}

An artificial neural network (ANN) is a "massively parallel distributed processor made up of simple processing units, that has a natural propensity for storing experiential knowledge and making it available for use" [Haykin, 1999; pg. 2]. The ANN closely resembles the human brain because it, first of all, acquires knowledge from its environment and secondly, it has interneuron connections that are used to store the acquired knowledge [Haykin, 1999; pg. 6]. For example, an ANN can be likened to a child learning the difference between cats and dogs under the supervision of his/her parents. The ANN learns the relation between input variables, for example size, fleece, voice, behaviour, and output variables, in this case cat or dog. After this learning period, also called "training", the ANN can accurately "predict" the output (either cat or dog) of any subsequent case presented to it [Zernikow et al., 1998].

The human nervous system is a three-stage system. The brain receives information, perceives it and then makes appropriate decisions. Information flows along a transmission route and in a forward direction. First, receptors convert a stimulus from the body or the environment into electrical impulses that go to the brain. Next, the brain makes a decision and finally, the effectors convert the impulses into a response or system output. The output can be sensed by the receptors again, essentially creating a feedback loop [Haykin, 1999; pg. 6].

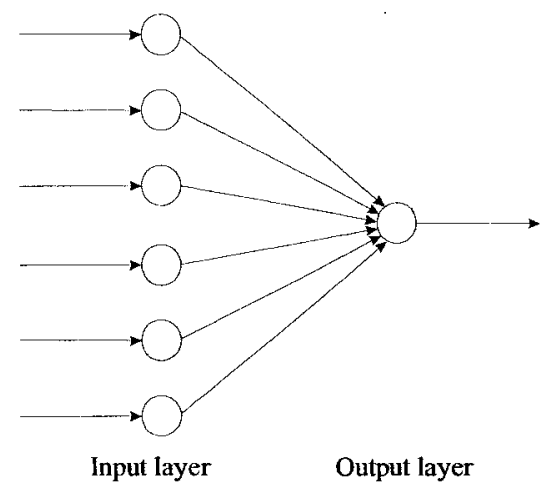

Figure 2-1: Basic ANN Architecture [Rybchynski, 2005] 
The ANN, modeled after the brain, is essentially a network of building blocks, called neurons or nodes, structured in layers. The most basic ANN architecture is formed by an input layer consisting of several nodes, connected to an output layer consisting of one or more output nodes as in figure 2-1. The nodes are connected by weighted links or synapses, and the network uses an adder to sum the input signals at the output layer and a transfer function or activation function that limits the value of the output as seen in figure 2-2. It should be noted that although the transfer function shown in figure 2-2 is a step function, other functions are commonly used, such as the sigmoid function which is used in this research. During training, the network is presented with inputs and known outcomes. The weights of the links are adjusted based on the relationship identified between the inputs and outcomes in the training set. Once trained the ANN can be used for prediction on a new set.

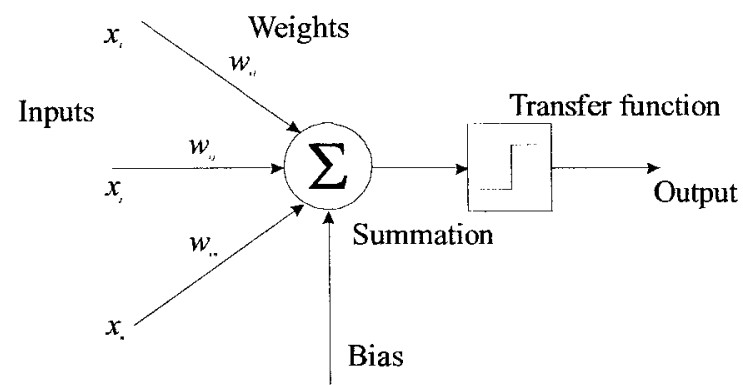

Figure 2-2: ANN with weighted inputs, summer and activation function at output

[Rybchynski, 2005]

There are three possible architectures for the ANN:

1. Single layer feed forward network: This architecture consists of only an input layer and an output layer as shown in figure 2-2. It is also called a single layer network, since no computation is performed at the input layer. Networks with no hidden layers are inherently linear and can only solve linear problems [Penny \& Frost, 1996].

2. Multilayer feed forward network: This structure consists of hidden layers inserted between the input and output layers as seen in figure 2-3. The 
addition of a hidden layer allows the network to solve more complex, nonlinear problems but requires additional training time [Penny \& Frost, 1996].

3. Recurrent network: This architecture involves at least one feedback loop. The addition of feedback in the network greatly increases its learning capability and performance [Haykin, 1999, pg. 23].

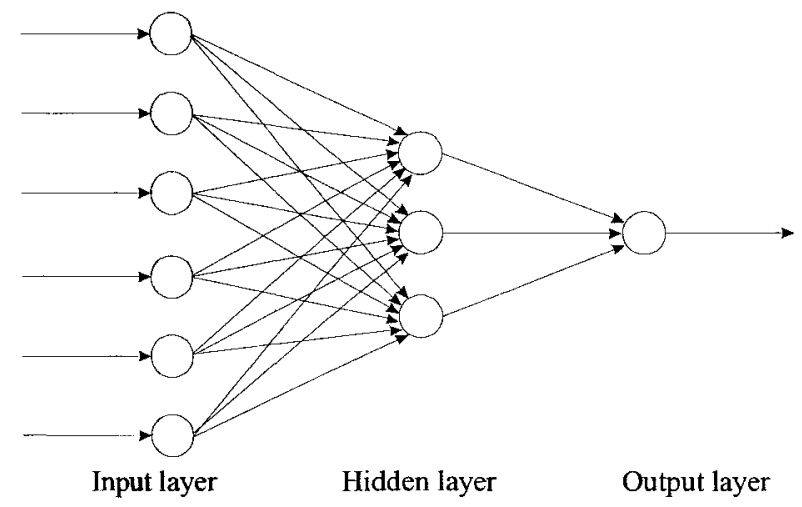

Figure 2-3: Multilayer feed forward ANN [Rybchynski, 2005]

As yet, there has been no substantial evidence that more than one hidden layer improves network performance. Having too few nodes in a network can lead to under fitting; this means the network has not learned enough and therefore, is incapable of classifying properly. If too many nodes are chosen, the network may be inefficient and a great deal of time may be lost in training. A network that has an optimal number of hidden nodes can provide good results with only one hidden layer for describing many functions [Hornik et al. 1989; Fausett 1994, pg. 328; Penny \& Frost 1996].

It should be mentioned that a linear problem solved using a linear neural network (with no hidden nodes) can also be solved using classic linear approaches such as the Wiener solution.

\subsubsection{Performance Measurement}

There are many different measures of ANN performance and criteria for stopping ANN training, some of which are presented in this section.

The conventional stopping criteria uses the minimum average squared error to stop the ANN training when the difference between the network's output and the known output falls below a predetermined value. However, there are many other stopping 
criteria presented in this section. If the training was to continue after the stopping point, overfitting may occur. Once the model has been finalized and only after it has been finalized, a validation set is used to test its performance. If a test or validation set is used in the training phase, it is considered 'cheating' since the model's performance on these sets are generally used as a measure of the model, and therefore, they cannot be used to develop the model. The following additional stopping criteria and performance measures are explained in this section: constant predictor (CP), correct classification rate (CCR), average squared error (ASE), receiver operating characteristic (ROC), log sensitivity index, sensitivity, specificity, confusion matrix, and weight elimination. The log sensitivity stopping criteria is implemented in the ANN tool used in this research.

\section{Constant Predictor (CP)}

The constant predictor (CP) is a simple statistical measure that classifies all cases as belonging to the class with the highest a priori probability.

For example, if an outcome A occurs $75 \%$ of the time and outcome B occurs $25 \%$ of the time, $\mathrm{A}$ is the predictor and the $\mathrm{CP}$ is $75 \%$. The a priori stats of a dataset are used as the benchmark because they remain constant regardless of which data mining method or performance measure is used [Frize et al., 1995].

\section{Correct Classification Rate (CCR)}

The number of cases correctly identified with respect to the total number of cases in the data set is called the correct classification rate or the CCR. The CCR measures the sensitivity to the frequency distribution of the outcome. The CCR is an accurate measure only if the desired outcome is above $15 \%$ of the total cases [Frize et al., 1998]. It is calculated as follows:

$$
C C R=\frac{T N+T P}{T N+F N+T P+F P}
$$

Equation 2-1: Classification Rate [Hohavi and Provost, 1998]

where TN is the true negative, TP the true positive, FN the false negative and FP the false positive. 


\section{Average Squared Error (ASE)}

The difference between the predicted value and its corresponding actual value, squared and averaged over all cases is the average squared error. It is commonly used as the stopping criteria and is calculated for one output node using the following equation:

$$
A S E=\frac{1}{2 n} \sum_{i=1}^{n}\left(\text { desired }_{i}-\text { actual }_{i}\right)^{2}
$$

Equation 2-2: Average Squared Error [Haykin, 1999; pg. 172]

\section{Receiver Operating Characteristic (ROC)}

The receiver operating characteristic (ROC) is a visual means to evaluate discriminatory ability of an ANN. It is a plot of the true positive rate against the false positive rate, which represents the probability that a randomly chosen positive case is classified with greater suspicion than a randomly chosen negative case. The perfect model has an ROC curve that approximates a step function that has $100 \%$ of the positive cases correctly identified. An excellent curve is shown in figure 2-4 as the curve labeled number 1. A good classifier is one that has a curve that lies above the 45 degrees diagonal also shown in figure $2-4$ by the curve labeled number 2 . Curve number 3 in figure $2-4$ is a worthless ROC curve that does not have the ability to classify data.

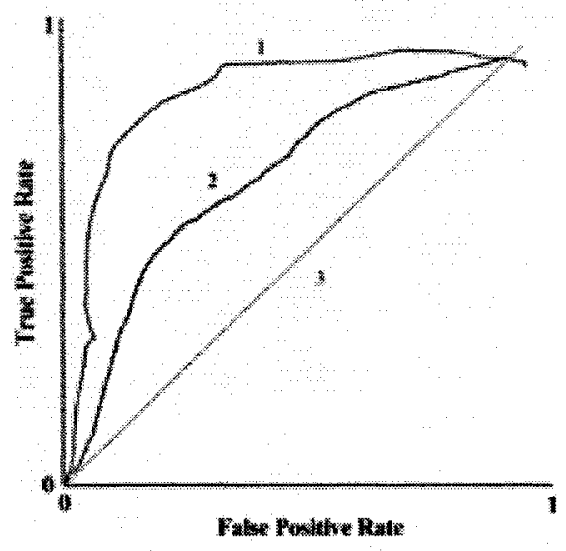

Figure 2-4: An excellent, good and worthless Receiver Operating Characteristic (ROC) curve [Pan, 2002]

The area under the ROC curve is a numeric performance measure that indicates the accuracy of the classification. The true negative and false positive can be calculated from the curve using the following equations: 


$$
\begin{gathered}
\mathrm{TN}=1-\mathrm{FP} \\
\mathrm{FN}=1 \text { - sensitivity }
\end{gathered}
$$

Equation 2-3: True negative and false negative calculations

[Hohavi and Provost, 1998]

The ANN returns a result as a real number between -1 and +1 that is then categorized into a positive or negative result based on a specific cut-off point in the continuous range of -1 to +1 . The cut off point is normally half way between the minimum and maximum returned from the ANN output function. To plot the ROC, the cut-off point is moved and the sensitivity and specificity at each cut off point is calculated. A worthless curve as the one shown in figure 2-4 indicates that the sensitivity and specificity both change significantly as the cut point moves, while a good curve indicates that the values are both high and do not change greatly as the cut point moves. The latter indicates that the model has achieved a stable state [Rybchynski, 2005].

\section{Sensitivity}

The sensitivity is a measure of how many positive outcomes were predicted correctly. It is calculated by the following equation, where TP is the number of true positive cases and $\mathrm{FN}$ is the number of false negative cases:

$$
\text { sensitivity }=\frac{T P}{T P+F N}
$$

Equation 2-4: Sensitivity [Penny \& Frost, 1996]

\section{Specificity}

The specificity is the true negative rate or the number of negative outcomes predicted correctly. It is calculated using the following equation, where TN is the number of true negative outcomes and FP is the number of false positive outcomes:

$$
\text { specificity }=\frac{T N}{T N+F P}
$$

Equation 2-5: Specificity [Penny \& Frost, 1996] 


\section{Confusion Matrix}

The matrix in table 2-1 is a visual representation of the composition of the actual and predicted outcomes from the positive and negative outcomes:

Table 2-1: A visual representation of the relationship between actual and prediction outcomes and true and false predictions

[Hohavi and Provost, 1998]

\begin{tabular}{|l|l|l|l|}
\cline { 3 - 3 } \multicolumn{2}{c|}{} & \multicolumn{2}{|l|}{ Actual Outcome } \\
\cline { 2 - 4 } \multicolumn{2}{c|}{$\begin{array}{l}\text { Negative } \\
\text { Outcome } \\
(-1 \text { or } 0)\end{array}$} & $\begin{array}{l}\text { Positive } \\
\text { Outcome (+1) }\end{array}$ \\
\hline $\begin{array}{l}\text { Predicted } \\
\text { Outcome }\end{array}$ & $\begin{array}{l}\text { Negative } \\
\text { Outcome (-1 or } \\
0)\end{array}$ & $\begin{array}{l}\text { True Negative } \\
(\text { TN) }\end{array}$ & $\begin{array}{l}\text { False Negative } \\
\text { (FN) }\end{array}$ \\
\cline { 2 - 4 } & $\begin{array}{l}\text { Positive } \\
\text { Outcome (+1) }\end{array}$ & False Positive (FP) & $\begin{array}{l}\text { True Positive } \\
\text { (TP) }\end{array}$ \\
\hline
\end{tabular}

Table 2-1 shows how outcomes are categorized depending on the predicted and the actual outcome. For example, if an outcome was predicted to be negative (i.e. survival when predicting mortality) and the actual outcome was negative, the case is categorized as a true negative.

\section{Log-Sensitivity Index}

The stopping criteria used by MIRG is the log-sensitivity index. In this case, the $\log$-sensitivity index is a good measure of the network's classification ability when the desired outcome is less than $15 \%$ of the total cases (a rare outcome) [Frize et al., 1998]. The log-sensitivity index attempts to achieve the optimal sensitivity and specificity while favoring higher sensitivity. It is calculated as follows:

$\log$ _sensitivity_index $=-$ sensitivity $^{\mathrm{n}} * \log 10(1$-sensitivity $*$ specificity $)$

Equation 2-6: Log sensitivity [Ennett et al., 2002; Scales, 2001]

Studies show that using log-sensitivity index results in better classification outcomes that networks that are manually optimized [Scales, 2001]. 


\section{Sensitivity vs. Specificity}

As explained above the log-sensitivity index, as used by MIRG, gives more significance to the sensitivity over the specificity. This is because in most datasets the less frequent outcome is the positive outcome and because it occurs less frequently, it is likely to be misclassified by the ANN. In this case, consider the less frequent outcome to be mortality of a neonate occurring $10 \%$ of the time, and the more frequent outcome to be survival of the neonate. If the CP of survival is used, $90 \%$ of the cases are classified correctly, but all positives or mortality cases are classified incorrectly. Therefore, it is imperative that the network give more significance to the correct classification of the positive cases, or the sensitivity measure.

For our research purposes, we also wish to ensure that specificity remains as high as possible so that the least number of infants that actually survive, will be falsely classified. At the same time, we wish to be able to accurately pick out which will die and therefore, a high sensitivity is also desired, but not at the cost of specificity. In this study, a specificity of at least $90 \%$ was maintained throughout the tests. A cut-off of $90 \%$ was chosen because in a study performed by Stevens et al., it was demonstrated that physicians were able to predict non-survivors with an accuracy of $90 \%$ [Stevens et al., 1993]. To be useful, the ANN tool should be able to predict non-survivors with at least as much accuracy as the physician or better. The highest sensitivity that can be achieved is desirable, although a sensitivity greater than $50 \%$ is deemed acceptable [Walker et al., 2002].

\section{Weight Decay and Weight Elimination}

Weight decay limits the size of a connection weight by stopping already large weights from further increasing. A weight decay constant determines how strongly weights are penalized. Weight-elimination reduces the small weights that have less effect on the network to zero. Weight decay and elimination work best on a large network structure, small initial weights and a small learning rate. Weight elimination is key in determining the minimum data set that is required to accurately predict a specific outcome [Ennett et al., 2003; McGowan et al., 1996]. 


\subsection{MIRG's ANN Software Tool}

The MIRG ANN tool was updated and improved by Dawn Rybchynski, using MatLab as a base [Rybchynski, 2005]. The ANN is a multilayer perceptron that uses a feed forward back propagation learning algorithm with the option of using weight elimination. ANN learning is based on the values of nine parameters: learn rate, learn rate increment and decrement multiplication factors, momentum and error ratio, lambda weight decay factor, lambda increment and decrement multiplication factors and weight scale for weight elimination functions [Frize et al., 2000].

The ANN tool can assess the performance of various architectures by varying the number of hidden layers and number of hidden nodes. The optimal ANN architecture is chosen as the final model and the weights and biases of the input variables from the epoch that gave the best performance are used as final model parameters. A prototype of the tool was used with a GUI created in MatLab for clinical trial in the NICU by Rybchynski for Qi as explained in section 2.4.4. This tool was designed specifically for predicting three outcomes from nine input variables as can be seen in figure 2-5.

Two limitations of the tool used by Qi were the slow speed of the tool and the inability to deal with continuous outcomes (as opposed to categorized outcomes). Rybchynski resolved these issues as well as adding other features to the tool in the most recent version. Unnecessary calculations and the display of redundant figures and output files was removed to improve the speed of the tool as well as the storage space required for its execution. In addition, functionality was added so that a number of structures chosen by the user could be modeled and analyzed automatically by the tool as explained in section 3.2.3. A verification set has also been added in the most recent edition. [Rybchynski, 2005].

In the remainder of this section the methodology followed when using the ANN RFW will be explained.

\subsubsection{Data Preparation}

The values in the day 3 neonatal database were manually checked to ensure there were no outliers for the variables. In future versions of this tool that are to be used in the 
clinical environment, this data checking step should be built into the system so that incoming data is automatically checked for outliers.

The data was then normalized using the $\mathrm{z}$-score formula as follows:

$$
\mathrm{Z}_{\mathrm{n}}=\frac{\mathrm{X}_{\mathrm{n}}-\mathrm{X}}{3 \sigma}
$$

Equation 2-7: Normalization Equation [Olden \&

Jackson, 2002]

where $z_{n}$ is the normalized value of variable $x, x_{n}$ is the original value of the variable and $\mathrm{X}$ and $\sigma$ are the mean and standard deviation of the variable $\mathrm{x}$.

In order to use the ANN RFW, the data was split into three sets: $1 / 3$ was first set aside for the verification set. Of the remaining data, a $2 / 3$ portion was used as the training set and the remaining $1 / 3$ was used as the test. From the verification set, ten verification sets were created using the bootstrap method, which is a method that uses resampling with replacement from an original data set. Each verification set had the same number of cases as the original verification dataset and yet each set was distinct because it contained duplicates of certain cases.

Verification data allows the network's performance to be verified on 'unseen' data, that is, data not used for training or testing. A verification set is necessary because the test set is used by the ANN RFW to provide feedback to the network and therefore, it cannot be used to give an accurate measure of the network's performance. After training is performed in one epoch, the weights and parameter settings obtained from training are run on the test data in the next epoch to ensure that the performance of the test set has also improved. If the performance of the training set improves but the performance of the test set does not over-generalization of the training data has occurred. But, if the performance of the test set also improves, training continues in the next epoch and this is repeated until the performance of the test set ceases to improve. Since the test set provides feedback to the network, using it as a final measure of the network's performance would constitute 'cheating'. It is for this reason that an 'unseen' dataset is used as the final measure of the network's performance after the training and testing phases of the network are complete. 


\subsubsection{Resampling}

MIRG has developed the technique of resampling or doping, first attempted by Ennett to increase the sensitivity of the network. This is done by artificially increasing the lower represented population, thereby changing the a prior statistics of the dataset. Ennett attempted this on non-survivors of a CABG (coronary artery bypass grafting) surgery database, who made up only $3.7 \%$ of the total number of cases in the database. Ennett discovered that the network would often classify everything belonging to the highest a prior probability when the positive case representation was between 8 and $15 \%$ and below 8\%, Ennett found that the network failed to learn the trends of the CABG database. A training set that was resampled to $20 \%$ mortality rate gave a significantly higher sensitivity without compromising specificity. Weight elimination was also discovered to increase the sensitivity. Ennett also concluded that predicting incorrectly that a CABG patient would not survive (a false negative case) is worse than foretelling survival when a patient dies (false positive case). Therefore, a high specificity (true negative predictor) is more important than a high sensitivity (true positive predictor) although optimizing both would be ideal. [Ennett et al., 2000; Ennett et al., 2003; Ennett, 2003]

Ennett resampled the dataset by maintaining the total number of cases in the training set constant. She added the non-survivors (negative cases) until the desired 80 20 ratio was obtained. Because the total number of cases in the day 3 neonatal dataset is already considerably small (compared to admission day dataset and other medical databases), this method was used in which the mortality cases were duplicated until a resampled ratio of $20 \%$ mortality was achieved.

\subsubsection{Network Parameters}

In a back propagation learning algorithm, as the one implemented in this tool, the connection weights and biases of the neural network are calculated backwards from the output to the input layer. The weights and biases of the network are changed depending on the error calculated from the network's output. A descending gradient search is used to search the error function for the global minimum or the lowest error across the entire function as opposed to a small valley in the function (local minimum) that may be 
mistaken for the global minimum. There is no guarantee that the algorithm will actually arrive at the global minimum, although this would be ideal. It is more likely that a local minimum will be attained that is fairly close in proximity to the global minimum.

The ANN RFW uses nine parameters to calculate and adjust the error of the network: learning rate, learning rate increment, learning rate decrement, lambda, lambda increment, lambda decrement, weight scale factor, momentum, error ratio. These parameters are the same ones that were in the older versions of the tool except that they were manually adjusted in the older versions and are automatically adjusted in the newest ANN RFW. The idea of automating the parameters using a divide and conquer algorithm was first proposed by Frize et al. in 2000 [Frize et al., 2000]. The following is a brief overview of the nine parameters the role each plays in neural network learning.

\section{Learning Rate $(l r, l r$ inc, $l r$ dec)}

The learning rate is the speed at which the network attains the minimum error value. If the learning rate is set too high, it may oscillate around the global minimum and if it is too small it may take too long to converge. The learning rate should be set so that it is small enough to ensure convergence but large enough that it may do so within an acceptable time [Frize et al., 2000].

Learning rate increment and decrement are the values by which the learning rate is changed.

\section{Lambda or Weight Decay Constant (lambda, lambda_inc, lambda_dec)}

The weight decay constant determines how strongly the weights are penalized. It limits the size of the connection weights by penalizing large weights by stopping them from increasing, thereby reducing the variance of the weights in a network [Frize et al., 2000].

Lambda increment and decrement are the incremental and decrement values, respectively. The values of lambda, lambda_inc, lambda_dec, and wnot (weight scale factor) are only used by the tool if the weight elimination option has been turned on. In the ANN RFW, the weight decay and weight elimination are either both on or both off. [Rybchynski, 2005] 


\section{Weight Scale Factor ( $w$ not)}

The weight scale factor defines the size of large and small weights. Weights that are smaller than the weight scale factor are forced to zero so that they are removed from the network. Weights larger than the weight scale factor are not eliminated but may be penalized using the weight decay constant [Frize et al., 2000].

\section{$\underline{\text { Momentum }}$}

The momentum value is a proportion of the previous weight change value that is added to the new value to give the algorithm some "momentum" so that it does not get caught in local minima. It allows the network to ignore small features in the error surface, acting like a low-pass filter [Frize et al., 2000].

\section{Error Ratio}

The error ratio value controls how the backpropagation makes adaptive changes in the learning rate, weight-decay constant and the momentum term as can be seen in the pseudocode of equation 2-8. The error ratio is used to check the error from one epoch to the next, with the error ratio value being the improvement required for the training to continue. The training set error from the current epoch is denoted by TSSE and the error from the previous epoch is denoted by SSE. If the network has improved (meaning that the error has decreased), the learning rate $(l r)$, lambda $(\lambda)$, and momentum $(m)$ are changed in the next epoch.

$$
\begin{aligned}
& \text { if } \mathrm{TSSE}>\mathrm{SSE} * \text { err_ratio } \\
& \mathrm{lr}=\mathrm{lr} * \mathrm{lr} \_\mathrm{dec} \text {; } \\
& \lambda=\lambda^{*} \lambda_{-} \text {dec; } \\
& \mathrm{m}=0 \text {; } \\
& \text { else if TSSE }<\text { SSE * err_ratio } \\
& \operatorname{lr}=1 \mathrm{r} * 1 \mathrm{r} \text { inc; } \\
& \lambda=\lambda^{*} \lambda_{-} \text {inc } \\
& \text { end }
\end{aligned}
$$

Equation 2-8: Adaptive ANN changes using the error ratio [Ennett, 2003] 
The nine parameters described above are optimized automatically in the ANN RFW. The ANN includes a range for each parameter, in which the tool searches for an optimal value using a divide and conquer algorithm. The ranges are set to values that were found to be appropriate in previous MIRG research. The new software attempts to find a midpoint within the range and compares the performance of the new value of the parameter with the previous performance. If the new value is better, it is carried forward as the new starting point and the divide and conquer is done again. When the performance ceases to improve, a stagnant point has been reached and the parameter has been optimized. This is done separately for all nine parameters and is done automatically by the tool [Frize et al., 2000].

The new ANN tool is able to more quickly arrive at the optimal parameter saving time and user supervision. It is also less important that the initial parameters be chosen carefully as they are changed within the first few iterations [Rybchynski, 2005].

\subsubsection{Network Structure}

Medical data is non-linear in nature and therefore, this tool can more easily find the optimal non-linear structure than the manual trial and error method required with the old tool [Frize et al., 2001]. In many clinical research tests, a single hidden layer (3-layered network) is used to define the non-linearity of the medical data being studied. Ibrahim-Swailhum, a former MIRG student, found that a linear structure was not able to predict mortality on perinatal data, while non-linear structures were able to [Ibrahim-Swailhum, 2005]. Ennett developed the MIRG neonatal mortality model using a linear network structure, but mentions that a 3-layered or non-linear network would have given better performance [Ennett, 2003].

Any function can be modeled using a number of hidden nodes in one hidden layer [Hornik et al. 1989; Fausett 1994, pg. 328; Penny \& Frost 1996]. Therefore, this tool attempts a linear structure (a single-layer network with no hidden nodes) and structures with a hidden layer that vary in the number of hidden nodes from 0 to $2 n+1$ nodes, where $\mathrm{n}$ is the number of inputs to the network.

There have been several theories as to how many hidden nodes are optimal for a given number of inputs, outputs and number of training cases. A common method is to 
set the number of hidden nodes to $2 \mathrm{n}+1$, where $\mathrm{n}$ is the number of inputs. This is also the method used to determine the upper limit of hidden nodes attempted by the ANN RFW [Rybchynski, 2005]. Using this method, the ANN RFW attempts from 0 to 19 hidden nodes for a 9 input variable dataset such as the SNAPPE-2 dataset.

Other theories that are mentioned by Ibrahim-Swailhum, include Masters' method in which the number of hidden nodes is defined by the following equation:

$$
d=\sqrt{i * o}
$$

Equation 2-9: Masters' method [Masters, 1993; pg. 177]

where $d$ is the number of hidden nodes, $i$ is the number of input nodes and $o$ is the number of output nodes

Using this theory, a 9 input variable dataset for predicting one outcome would require only 3 hidden nodes. Masters also states that $50 \%$ and $75 \%$ of the number of input nodes can be used as a selection method [Masters, 1993].

There is also Livingstone and Manallack's method which considers the number of training cases to compute the number of hidden nodes:

$$
d=\frac{m * o}{w}
$$

Equation 2-10: Livingstone and Manallack's method [Livingstone and Manallack, 1993]

where $d>3$ to ensure good generalization, $\mathrm{m}$ is the number of training cases, $o$ is the number of network outputs and $w$ is the total number of network weights. So, for a network with 3000 training cases, the network should not have more than 1000 weights. For 9 input variables, this works out to 100 hidden nodes. Therefore, this method produces a very large number of hidden nodes because the datasets used in this research consist of large training sets. Ibrahim-Swailhum also states that large databases require less hidden nodes than smaller ones, because a neural network will have more difficulty finding the data relationships when given only a small number of cases. [IbrahimSwailhum]

For this research, structures with hidden nodes varying from 0 to $2 n+1$ are attempted as the ANN RFW has been set to perform by default. The structure that produces the best result will then be selected as the optimal network structure. 


\subsubsection{Structure Selection Criteria}

MIRG has adopted the use of the log sensitivity performance measurement as the stopping criterion for the ANN tool. Therefore, the ANN RFW uses the log sensitivity to find the parameter values that give the best performance and the structure of the 0 to $2 \mathrm{n}+1$ structures that gives the best performance.

For every structure attempted, the epoch at which the highest log sensitivity was obtained is saved as the best test epoch by the tool. The performance of the network at this epoch is recorded and the weights, biases and nine parameter values at this point are extracted. The tool then runs the verification sets on the network using the optimal weights, biases and parameters to ensure the performance of the network can be verified on unseen datasets.

The ANN RFW chooses the best performing structure based on the highest log sensitivity achieved at the best test epoch. The best result structure defined by the ANN RFW was not necessarily the best structure for this research, since this research focuses on obtaining the highest sensitivity possible without compromising the specificity. Therefore, the structure that gave the highest $\log$ sensitivity was not necessarily the best when predicting mortality on neonatal data. For example, a structure that gave a sensitivity of $70 \%$ and specificity of $80 \%$ (a $\log$ sensitivity of about 0.25 ), was not preferred over a structure with $60 \%$ sensitivity and $90 \%$ specificity (a log sensitivity of about 0.20 ), although the ANN RFW would prefer the former structure because it gave a higher log sensitivity.

To implement a new structure selection criterion the stopping criteria of the ANN software was changed (in the stopCriterion.m file) so that it chose the parameters and structure of the network based on the best test log sensitivity while maintaining a specificity of at least $90 \%$. The specificity limit was set to $90 \%$ based on the study by Steven et al. in which it was found that physicians predicted mortality with $90 \%$ specificity and $68 \%$ sensitivity [Steven et al., 1994]. The goal of this research is to determine a model for day 3 mortality prediction that will ultimately be used in a clinical setting to predict mortality, the model should predict with an accuracy that is at least as good as the physician's. As already stated in section 2.1 .2 , a specificity as close to $100 \%$ 
as possible and a sensitivity above $50 \%$ is desired according to Dr.Walker, an expert of neonatology [Walker, 2003].

Although the stopping criterion of the ANN RFW was adjusted to suit the requirements of this research, the best overall structure found by the ANN RFW was not automatically taken as the best structure for this research. The following is a criterion that was developed for selecting the best structure for this research from the results of the ANN RFW. For each of the structures chosen by the tool as the 'best structure':

1. The log sensitivity, sensitivity and specificity graphs were examined to make sure the network was able to converge. The graphs that oscillated considerably throughout or were flat throughout were discarded. Only the graphs that demonstrated that learning occurred were considered.

2. The best test epoch taken by the ANN RFW for the best structure was located on the $\log$ sensitivity graph to ensure that it was taken at a point where the graph had stabilized and was not chosen at an unstable point on the log sensitivity graph. It was also ensured that the best test epoch was not before epoch 100 as this would not give sufficient time for the network to converge accurately.

3. The ROC was examined to ensure that it was acceptable (see section 2.1.2) and that it was a full curve. A half-finished ROC curve as was found in some cases, indicates the network failed to complete learning.

4. The $\log$ sensitivity, sensitivity and specificity values of the 10 verification sets were averaged. A good verification set performance would indicate that the network did in fact learn and did not generalize or over-fit the data. Some verification sets gave all 0 's meaning the network had not converged and therefore verification could not be performed.

\subsubsection{Extracting the Weights}

The weights of the ANN were used in the CBR as explained in section 2.3. Ennett had developed a mortality model using only a linear (2-layered) network. It was easy to transfer the single set of weights that were extracted from the linear network to the CBR directly. Rybchynski's addition of non-linearity to the ANN tool initiated the need for a new method of extracting the weights. Therefore, Rybchynski investigated the 
Garson-Goh method of extracting weights from a three-layered network.

Rybchynski found the following two weaknesses in Garson-Goh's method [Rybchynski, 2005]:

1. The use of absolute value loses some of the interaction between weights of the same input node. Weights of a positive and negative magnitude should have cancelled each other out, but are counted twice by the Garson-Goh method.

2. A second and more significant error of the method was that the weight of the hidden node to output node for each hidden node cancelled out of the equation, so that this weight was eliminated from the calculation of the relative weights.

Rybchynski corrected these errors and came up with the following set of equations for extracting the relative weights [Rybchynski, 2005]:

1) Calculating the relative weighting of the hidden-output connection for the output node: $r w_{j k}=\frac{a b s\left(w_{j k}\right)}{\sum_{j} a b s\left(w_{j k}\right)}$

where $\mathrm{j}$ is the number of hidden nodes and $\mathrm{k}$ is the number of output nodes

2) Calculating the relative weighting of the input-hidden connection for each hidden node: $r w_{i j}=\frac{a b s\left(w_{i j}\right)}{\sum_{i} a b s\left(w_{i j}\right)}$

where $i$ is the number of input nodes

3) Multiplying each relative weighting input-hidden connection value by the relative weighting value of its connected hidden-output weight: $P_{i k}=r w_{i j} * r w_{j k}$

4) Sum the final relative weighting products: $S_{i}=\sum_{i} P_{i k}$

5) Divide the sum of the final relative weightings for each input node by the total of all relative weightings: $R I_{i}=\frac{S_{i}}{\sum_{i} S_{i}}$

,where $i$ is the number of input nodes, $j$ the number of hidden nodes, and $k$ the number of output nodes and where $\mathrm{w}_{i j}$ represents the connection weight between nodes $i$ 
and $j$.

Rybchynski also developed a MatLab program which requires the user to input the weights and biases of a 3-layered network in order to output the relative weights of the input variables. This program was used to extract weights from the 3-layered network structures for use in the CBR.

\subsection{Case Based Reasoner}

\subsubsection{Case Based Reasoner Theory}

The case based reasoner (CBR) is an extension of the knowledge-based system (KBS). It draws on past experience from a case base and is able to adapt a successful solution from the past to a similar problem. The CBR, in contrast with the KBS, uses reasoning instead of defined rules and is therefore, the method of choice when working in domains that have no concrete rules [Ennett, 2003].

The CBR uses a retrieval algorithm to find the most similar case in its case base. One example of a retrieval algorithm, and the one that is used in this research is the nearest neighbor algorithm. This algorithm calculates the weighted distance between the cases in its repository and the target query and the case with the smallest distance from the query case is chosen as the closest. The $\mathrm{kNN}$ algorithm calculates the distance by plotting all points in a n-dimensional space. A weighted $\mathrm{kNN}$ algorithm takes into account weights assigned for each input variable of the case. Input variables with greater weights are plotted along an elongated axis forcing the difference in values along the axis to be more important. Conversely, input variables with lower weights are plotted along a shrunken axis on the plot [Cotea, et al., 2003; Rybchynski, 2005].

An advantage of the $\mathrm{kNN}$ algorithm is its ability to deal with missing values in the query. It is able to find the closest matching cases even though the missing value cannot be plotted. The $\mathrm{kNN}$ is used in this research to impute missing values of the variables recorded in the NICU database. The weights for each variable are simply used to determine their importance when the $\mathrm{kNN}$ performs its matching algorithm.

A weakness of the $\mathrm{kNN}$ is that it requires a predefined knowledge of the inputs of the query and their associated weights [Rynchynski, 2005]. A second weakness is the 
exponential increase in computation time of the $\mathrm{kNN}$ with a larger number of cases. Therefore, it is most efficient when used with smaller case bases [Ennett, 2003; Frize et al., 1993].

\subsection{MIRG's CBR Software}

The CBR initially used by Ennett was the Easy Reasoner by Haley Enterprises, which was customized for MIRG research by a team at the University of New Brunswick. It employed the nearest neighbour algorithm in a two step process. First, a pre-query processing was performed in which an index was developed to identify the closest matching cases depending on the statistics of the database. Secondly, a query processing was performed in which the distance between the match cases and the query cases were calculated and the smallest one was taken.

This tool was difficult to access and soon became obsolete. It was replaced with a replicate that had been written by two fourth year MIRG students. The new tool was a JAVA program that used an ODBC connection to a multi-table Access database [Cotea, et al., 2003]. Originally, it was only able to match a single case to a database of existing cases and it was later improved to impute several cases at once with the mean of the closest matching cases [Rybchynski, 2005].

The current version of the CBR, known as the kNN used by MIRG, implements a weighted matching algorithm that allows external sources to provide weights for each input variable, so more significance can be given to certain variables when cases are matched by the $\mathrm{kNN}$. To obtain the weights of the input variables, the $\mathrm{kNN}$ is used in conjunction with a second data mining tool.

The kNN tool can provide several functions including the following [Rybchynski, 2005]:

1. Matching closest cases for inspection by a clinician: This function allows the ten closest matching cases to be viewed and analyzed by the clinician. The relevant inputs and corresponding weights have been defined with the assistance of a domain expert. [Frize et al., 1993]

2. Imputing missing values into patient cases: This function imputes the mean of the ten closest matching cases into the missing value of the target case. Imputation can be done in two ways: vertically to impute missing values to increase the 
number of complete cases or horizontally to impute missing variables to increase the number of variables for each case. The weights and inputs to be used for imputation have been defined with the assistance of a domain expert.

3. Extending datasets to include missing minimum data set variable when merging databases to create a multi-source test set: This function allows a dataset missing variables to impute the missing values using horizontal expansion as described above. The variables included in the data set and their corresponding $\mathrm{kNN}$ weights need to be set.

4. Predicting outcomes: Since the ANN cannot deal well with continuous outcomes without the continuous input and output being categorized, the $\mathrm{kNN}$ can be used instead to predict outcomes by outputting the mean of the continuous outcome for the ten closest matching cases. The inputs and relative weights must be predefined for each outcome.

\subsubsection{The Need for Imputation of Missing Values in NICU Data}

One way to deal with missing values in a database, would be to simply ignore the incomplete cases and to consider only the complete cases. Ennett observed that the mortality rate for infants with a complete set of variables was higher $(9.7 \%)$ than the mortality rate for the whole database of infants $(3.7 \%)$. Therefore, the cases that have no missing values are the sickest infants of the population [Ennett et al., 2001]. Any model built on the complete cases only would not be applicable to the entire population [Ennett, 2003]. Therefore, the accurate imputation of missing values is an essential step in finding a new mortality model.

\subsection{Neonatal Intensive Care Unit}

The NICU has undergone drastic changes due to the advance of science and technology in the field, over the last three decades. There has been a $45 \%$ decrease in very low birth weight babies between years 1989 and 1995. Obstetric care has increased due to improved ultrasound, more aggressive antenatal corticosteroid treatment (a steroid given to the mother to mature the fetus' lungs), delay of delivery using an agent called tocolytis, and an increase in caesarean sections to improve on the survival outcome in the 
case of a complicated delivery. NICU care for the newborn has improved with the introduction of surfactant replacement therapy (used to keep the lungs open) and different modes of mechanical ventilation [Richardson et al., 1998].

Richardson et al. studied the question of whether mortality rates had improved over the two cohorts studied (1989 to 1990 and 1984 to 1985) because of "better babies", meaning obstetric care has improved the condition of infants before they are admitted to the NICU, or "better care" in the NICU after the infant is admitted. It was concluded that both played a part in the decreased mortality rate. Although, there were just as many very low birth weight or VLBW (less than 1500g) infants in the second cohort as the first, the illness severity score of the VLBW infants was better, indicating "better babies" due to better obstetric care. It was also noted that for similar birth weight and severity score in the second cohort, there was still a better chance of survival, indicating "better care" [Richardson et al., 1998].

This section will look at several mortality models that have emerged over the past decade. It will also briefly describe the Canadian Neonatal Network, which was the source of the research data used in this study. This section also covers the use of ANN and CBR technology in the NICU and the temporal issues associated with NICU predictions.

\subsubsection{Mortality Models}

There are several models used to assess severity in neonates. Some of the more commonly used models are discussed in this section.

\section{Score for Neonatal Acute Physiology (SNAP)}

The score for neonatal acute physiology or SNAP was developed to fulfill a need for a standardized measure of illness severity in neonates. A score was necessary due to the wide variations found in the mortality and morbidity rates across several NICUs [Richardson et al., 1993].

SNAP assesses the degree of deviation from the physiological normal across several routine laboratory observations. The scoring system involves the clinician assigning a score from 0 (normal) to 5 (life-threatening) for each of the 26 items in the scoring system. The list of the SNAP variables and the scoring ranges can be found in 
appendix A. The SNAP assessment takes about 5 to 15 minutes to calculate depending on the patient and physician [Richardson et al., 1993].

SNAP was validated with positive results on admissions across 3 NICUs for an 11 month period. A clear degradation in mortality rates for increasing severity score within each birth weight stratum was observed. The score was observed to also parallel physician estimates of mortality risk and actual hospital mortalities. SNAP was found to be highly correlated with measures of resource use, therapeutic intensity, nursing workload and the length of stay (LOS) [Richardson et al., 1993].

The SNAP score is assessed usually within the first 24 hours of admission in an attempt to ensure the illness severity assessment is least contaminated with the infant's response to therapy. However, the 24 hour window was later identified as being too broad of a time frame and the contamination of the score with therapeutic response was recognized as a limitation of SNAP. A second limitation of SNAP was its long list of variables and therefore, its complexity in calculations [Richardson et al., 1993]. This issue was also addressed and corrected in the development of SNAP-II. The list of SNAP-II variables can be found in appendix A.

\section{SNAP with Perinatal Extension (SNAPPE)}

SNAP with perinatal extension or SNAPPE was then created to combine the physiological mortality risks identified in the creation of SNAP with the mortality risks commonly associated with premature infants. The three perinatal variables added were birth weight, Apgar score at 5 minutes and small for gestation age. The scoring ranges for these variables can be seen in appendix A [Richardson et al., 2001].

The Apgar score is a quick initial assessment of a newborn's condition taken at one, five and ten minutes after birth. A score of 7 to 10 is normal, 4 to 6 may require some minor interventions and under 4 indicates the baby may require immediate resuscitative measures. The physiological signs measured and the scoring methodology is shown in appendix A [Ennett, 2003; Dr. Apgar, 1953].

\section{SNAP-II and SNAPPE-II}

As indicated earlier SNAP scoring created in 1993, was cumbersome because of the number and complexity of its variables. In 2001, Richardson et al. disclosed their 
attempt at creating a second generation scoring system that would be simpler and more reliable. Furthermore, Richardson et al. wanted a new system that was specially adapted for mortality prediction and not just a general indicator of the illness severity for the triage of mildly ill patients. It was also desired that the score reflect the actual mortality risk and not the clinician's predicted risk. The significant improvement to NICU care in the 90 's spawned the need to re-calibrate the scoring system to reflect the more recent mortality risks.

To create the new model, the variables of the 37 SNAP variables that had no relation with mortality were removed using logistic regression, leaving only 6 variables. The variables of the SNAP-II system were the ones that were more extreme and therefore the best indicators of mortality. The data collection window was shortened from 24 hours for SNAP to 12 hours for SNAP-II, like in the CRIB scoring method. Any further shortening of the window is thought to diminish the data quality and possibly lower the predictive accuracy of the scoring system [Richardson et al., 1993].

SNAPPE-II was designed for patients less than $1500 \mathrm{~g}$ and included all six SNAPII variables in addition to the three perinatal variables used in SNAPPE: birth weight, Apgar 5 score and small for gestational age. For infants with birth weight greater than $1500 \mathrm{~g}$ it was found that birth weight has little additional prediction power on the risk of mortality. For this range group, the prediction of mortality is well captured by the scoring done in the first 12 hours of life. In contrast, the prediction of mortality for the infants in the birth weight group less than $1500 \mathrm{~g}$ is inherently difficult. Many events that occur later after the first 12 hours, such as sepses, are common causes of mortality and these cannot be foreseen by the scoring system [Richardson et al., 1993].

\section{Clinical Risk for Babies (CRIB)}

The clinical risk for babies score (CRIB) was developed for infants less than $1500 \mathrm{~g}$ or with a gestational age of less than 31 weeks. It was developed before the surfactant therapy became widely used in the 1990s. CRIB uses data collected in the first 12 hours after birth [INN, 1993]. 
Neonatal Therapeutic Intervention Scoring System (NTISS)

The NTISS was developed using data between November ' 89 and September ' 90 . It was based on the adult-ICU score called Therapeutic Intervention Scoring System (TISS) [Grey et al., 1992].

\section{Comparison}

Ennett of MIRG developed a 13 variable model that slightly outperformed the SNAPPE-II model on predicting mortality using admission day NICU data. The classification rate of Ennett's model was $96.8 \%$ when specificity was optimized and $84.2 \%$ when sensitivity was optimized, while the SNAPPE-II model's CR rates for the same test were $95.9 \%$ and $70.8 \%$ respectively [Ennett, 2003 ].

\subsubsection{Canadian Neonatal Network Database}

The data used by MIRG for neonatal research comes from the Canadian Neonatal Network (CNN) database. The CNN is a compilation of data from 17 neonatal intensive care units (NICU) across Canada from January 8, 1996 to October 31, 1997. It contains data on 20,488 cases collected on days 1 (admission), 3, 14 and 28 (or discharge). The data collected on day 1 was collected within the first 12 hours of admission. Infants in a NICU for less than 24 hours were excluded from the database to avoid the impact of different admission protocols of different hospitals [Ennett, 2003; CNN, 1993].

Neonatal mortality is defined by the World Health Organization as death occurring before 28 days of age. The CNN did not perform follow-ups for discharged infants, therefore only the infants who died during their stay in NICU and within 28 days were considered in this study. Infants near the point of death, also known as moribund infants, were excluded from the study as well since they do not receive aggressive therapy.

Of the 20,488 cases on day 1 , the cases missing output (mort) data or any of the perinatal extenstion variables deemed important (bthwt, sga or apgar5), were excluded. Thus, the complete dataset for day 1 infants consisted of 19,427 cases [Ennett, 2003]. A similar method was used to prepare the day 3 database so that the original set of 14,261 cases was reduced to 13,871 . 
The database recorded a large number of variables including the nine SNAPPE-II variables. MIRG used this data to predict infant mortality, number of hours on ventilator, length of stay in the NICU and other potential complications. The CNN data and its description is given in appendix B. There was also an extension of the CNN known as the Evidence-based Practice Identification and Change (EPIC) database. It contained 59 cases of data on premature infants from CHEO in 2002. Since it contained the same variables as the CNN database, it was used as a verification set in previous MIRG research performed on the CNN database [Qi 2005].

\subsubsection{The use of ANN in the NICU}

ANN technology, designed as a CDSS, has an increasingly growing presence in the NICU. Its performance has been compared to older methods of prediction such as mortality models, logistic regression (a statistical technique that predicts the probability of an outcome using independent variables) and clinician prediction. The following describes how the performance of ANNs in the NICU is showing promise.

\section{ANN vs. Logistic Regression}

Zernikow et al. and Ambalavanan et al. compared the performance of logistic regression to that of an ANN in the prediction of mortality in the NICU. Zernikow et al. observed that the ANN was better at recognizing the subtle and the non-linear associations between input variables and mortality that were not detected by logistic regression analysis. Zernikow et al. concluded that the $\mathrm{ANN}$ is a good prediction tool for most preterm infants but current models showed a significant number of failures making it unsuitable for individual treatment decisions.

Ambalavanan et al. also compared logistic regression to ANN prediction but came to a different conclusion. His study found that the performance of the logistic regression method was similar to the ANN and both were reasonably accurate, although the ANN would be preferred in the case of a large data set. Ambalavanan et al. came to the conclusion that analyzing specific variables better predicted mortality than analyzing the complete set of variables. The most significant variables in predicting mortality were found to be birth weight and the Apgar 5 score [Ambalavanan et al. 2001]. 
Other similar studies have been performed comparing ANN prediction power to that of logistic regression. Another such comparison was done for predicting time to extubate neonates on mechanical ventilation. The ANN outperformed logistic regression and clinician prediction [Mueller et al., 2004].

\section{ANN vs. Clinician Prediction}

An issue of great importance in the NICU, especially given the recent increase in survival rates of neonates, is the physician's ability to predict the mortality and morbidity (freedom from handicap in the case of survival) of a neonate. Physicians generally base their prediction on a few variables that can be quickly and easily obtained to give the most accurate prediction. The variables gestational age and birth weight are a common basis for predicting mortality [Fowlie et al., 1998]. In a study done by Qi, the top three prediction variables found among six physician's at a NICU were birth weight, lowest pO2:FiO2 ratio in the first 12 hours, and gestational age [Qi, 2005].

Although, the ability for a physician to predict outcomes has been observed to be fairly good (50\% sensitivity and $90 \%$ specificity according to Stevens et al. [Stevens et al., 1993]), studies show that it alone is not enough to decide an individual's treatment plan. According to the theory of reasoned action, the physician's knowledge and experience influence their decision on the course of treatment for the patient. It is important for a physician to have accurate estimations and to be up-to-date with the current statistics and trends in the NICU.

Morse et al. found that pediatricians and obstetrician's significantly underestimated both survival and freedom of handicap in preterm infants although the predictions were more accurate for gestational ages above 36 weeks. Underestimating survival and freedom from handicap were associated with the physician being less likely to pursue therapeutic interventions [Morse et al., 2000]. Wilson et al., on the other hand, found that pediatricians are the most accurate at predicting prognosis compared to other physicians, and are better at predicting mortality than they are at predicting morbidity [Wilson et al.]. It was also found that the more confident physicians are in their prognosis, the more likely they will hypothesize on the extremes ( 0 and $100 \%)$, whereas physicians who are less sure give a 50-50 prediction [Marcin et al., 2004]. 
The likely cause for underestimation is the failure of physicians to be up-to-date with the latest NICU statistics in which mortality and morbidity rates have fallen significantly over recent decades [Morse et al., 2004]. It is hopeful that an artificial intelligence tool in the NICU will act as a reliable aid for the physician in estimating survival and therefore, will encourage the use of interventions that may improve the quality of care.

Qi hypothesized that the prediction capability of an ANN would be superior because of its ability to quickly analyze large amounts of data and to derive relationships in the data. The ability of a physician to predict an outcome, on the other hand, relies on the experience of the physician. The physician is limited in the number of patients and cases they are able to experience. Although modern record keeping makes it easier for a physician to recall and apply a past case, the amount of data a physician is able to process is nothing comparable to the ANN. Qi added that the ANN can be updated easily. If a new treatment emerges that may influence the outcome, it may easily be added to the ANN's input parameter list, whereas the physician requires more time to adapt to the impact of new ideas and technologies in the field [Qi, 2005]. In a study on predicting extubation using ANN, it was noted that the clinician would much rather err on the side of extubating later than too early [Mueller et al., 2004]. As discussed in section 2.1.2, clinicians emphasize the correct prediction of mortality more than the correct prediction of survivors out of caution. Another benefit of the ANN is that it is able to differentiate between the two predictions, as the clinician does, and can ensure a higher prediction on the side of non-survival by keeping a high specificity. 


\subsection{Literature Review and Relevant Work}

\subsection{Temporal Issues in the NICU}

Having described the NICU environment and how the use of ANN technology overcomes limitations of past prediction models, there are several issues in the NICU pertaining specifically to time. These issues have been studied in greater detail in past years given the changing NICU trends. This section will discuss the temporal issues of relevance to the NICU from various studies.

\subsubsection{Issues to Consider for Temporal Clinical Decision Support Systems}

Chittaro et al. identify the main duties of an intelligent monitoring system in a medical environment [Chittaro et al., 1997]:

1. must be able to acquire and exploit the data available to propose a diagnosis of the patient's state

2. must be able to filter the numerous alarms from monitors to indicate only those that require a human intervention

3. must be able to propose specific therapeutic strategies depending on the evolution of the patient's state

One such system is known as MARY for the hospital in which it was found: Maternity Hospital of Edinburgh. MARY is a computerized trend monitoring system that has been used for more than 10 years in the NICU. Physiological information is continuously collected from the bedside of the neonate and displayed in real time. The most commonly used feature found through a survey of the clinical staff was the simultaneous display of changes in more than one physiological parameter or trend monitoring. The trend monitoring feature allows changes to be detected to alert the staff of a potential alarm in cases where the clinician may not otherwise realize the danger, because physiological changes may be acute and unobvious. MARY was simply a system for gathering and displaying data and did not have any intelligence at the time the study was done. The clinical staff surveyed in the study recommended the system be endowed with some form of intelligence so it would be able to automatically interpret the 
data, summarize relevant events and generate warnings if the data exceeded a threshold value [Alberdi et al., 2000].

\subsubsection{Trends in Time of Death in the NICU}

A group from the University of Chicago performed research on the trends in NICU mortality. The group sought to compare the predictive power of severity scores as time passes. The three possible relationships between time and physiological signals (measured by the severity score) that were identified were the following:

1. The scores of patients who will ultimately survive, improve over time, whereas the ones who will ultimately die become worse over time.

2. Severity scores of both groups, the surviving and the non-surviving, converge making them indistinguishable from one another. This is under the assumption that the physiological disturbances in a high risk infant are transient and are treated during the ICU stay.

3. There is a U-shaped pattern of severity scores, where the scores of the two groups temporarily converge and with time, diverge again.

The group discovered that instead of non-surviving infants "declaring themselves", they "cloak themselves" by seeming less likely to die [Meadow et al., 2002].

In another study done by Meadow et al. to observe changes in mortality rates of extremely low birth weight infants (less than $500 \mathrm{~g}$ ) in particular, the mortality rate of ELBW infants rose approximately 4\% per year from the period of 1991 to 1997 and then remained steady from 1997 to 2001 . The smaller infants consistently made up $40 \%$ of the deaths. In 1991, more than half of the ELBW non-survivors had perished by day 2 but in 2001, by day 10 only half had perished. The average length of stay rose from 5 to 17 in this period. The main repercussions of these findings is on the waiting time of the parents. Previously, they were able to receive news about their child's response to treatment in only 2 to 3 days, whereas now they had to wait more than 10 days [Meadow et al., 2004]. Therefore, Meadow et al. found that there were more survivors over the last decade and that the non-survivors lived longer before their ultimate demise. 
Fowlie et al. discovered that $48 \%$ of the neonates who die before discharge do so in the first 72 hours. Based on this statistic, it was proposed that the mortality prediction model be made on data obtained after the third day of life as opposed to admission data as is the conventional method. Fowlie et al. found that the CRIB score obtained at 72 hours and the ultrasound scan at 72 hours were better predictors of death than gestational age and birth weight. Fowlie et al. also identify some of the common pathologies linked to death in neonates after day 3 , of which the top three were respiratory distress syndrome, intraventricular hemorrhage, and pulmonary interstitial emphysema [Fowlie et al., 1998].

The numbers obtained by Fowlie et al. were similar to those of Sankaran et al. who found that $40 \%$ of deaths occurred within 2 days of NICU admission, $50 \%$ within the first 3 days and $75 \%$ within 12 days. Only $10 \%$ of deaths occurred after the first month. Sankaran et al. differentiated the infants in the study into two groups, those of less than 500g (ELBW) and those upto and including $1500 \mathrm{~g}$ (VLBW). The mortality rate for the smaller group was $51 \%$ and for the bigger group was $2 \%$. The conditions associated with death for both groups were different as well. For infants greater than $500 \mathrm{~g}$, the most prevalent condition was outborn status (where an infant is outborn if it was born at a different hospital) and chromosomal or congenital anomalies, whereas in the smaller group, infection and hypoxic-ischemic emcephalopathy (caused by birth asphyxia) and small for gestational age were prevalent factors in death [Sankaran et al., 2002]. It was concluded that longitudinal monitoring of NICU mortality rates is important in improving the efficacy and efficiency of NICU care.

In a study done by Civetta et al. on an adult ICU, 372 surgical patients were assessed using the APACHE II scoring system upon admission and on days 2, 3, 5, 7, 14 and the day before patient discharge. Civetta wanted to determine if ICU admissions could be limited using the APACHE II score and if the scoring system could give an accurate outcome prediction. The duration of ICU stay, patient outcome and total hospital charges were also recorded for each patient. Of the 372 patients, 292 survived to hospital discharge and 80 died. The patients who had an admission day score of less than 10 (low risk patients) were divided according to the length of ICU stay so that there were two groups: patients with a stay of 4 days or less and patients with a stay of 5 days or 
more. In the group with a stay of less than 4 days, the mortality rate was $13.1 \%$ compared to the latter group with a mortality rate of $59.6 \%$. So, there was a significant increase in mortality with increased duration of ICU stay for APACHE scores less than 10. For patients with an admission day score of more than 24 (high-risk patients) the opposite was found: there was an increase in survival with increased duration of ICU stay.

Civetta et al. concluded that survival rates varied according to duration of ICU stay. Patients within the same range of APACHE II score but with different ICU stays had different survival rates. Civetta et al. explained that the scoring system is limited in its ability to predict unique events which often determine the patient's outcome, such as idiosyncratic reaction to drug or massive fatal pulmonary embolus that can occur a month after discharge. Another common cause of death in surgical patients, the sepsis syndrome (a systemic disease associated with the presence of pathogenic organisms and their toxins in the blood) cannot be identified by the admission day score if the organ system is functioning normally on the admission day. The results of the study indicate that therapy has a great affect on the outcome and variables other than the patient's physiological symptoms on admission must be considered to accurately predict patient outcome [Civetta et al., 1990]. Although Civetta et al. studied the adult ICU, these observations can also be applied to mortality prediction in the NICU.

\subsubsection{Temporal Effects on Physician Prediction and Confidence}

Meadow et al., in the study mentioned above, researched changes in predictive power of clinical intuitions with time. Mortality was predicted using both the SNAP score and clinical intuition. Three main groups were characterized by SNAP scores:

1. 19 of the 45 non-survivors died within the first 2 days with significantly higher SNAP values than those who died after day 2.

2. 22 of the 26 non-survivors who survived until day 3 had downward trend with final SNAP scores lower than their SNAP on day 1

3. only 9 of the 45 non-survivors had a U-shaped course where their SNAP scores initially fell but rose again before death.

The ROC curves for SNAP values on days between day 1 and day 10 were plotted. The area under the curve diminishes greatly from 0.84 on day 1 to 0.64 on day 10 
indicating that SNAP scores become progressively less predictive and sensitive as an index of an infant's death.

In the second part of the study using clinical intuition, referred to as "prediction profiles", to predict mortality outcome, most non-survivors had remarkably homogenous and accurate prediction profiles that predicted death. The second largest group had generally optimistic profiles but ultimately suffered rapid and unexpected deaths. The study also concluded that physicians and nurses have about the same prediction capabilities. In many instances where death was predicted, the infants ended up ultimately surviving. This is, according to the group, because of the NICU phenomenon that most infants survive - even the very sick ones. This is further demonstrated by the fact that of the $40 \%$ of survivors that had been profiled to die, fewer than $20 \%$ were neurologically normal at the end of the first year. This fact also shows that although death is difficult to predict, the clinicians were quite good at predicting "neurological death" [Meadow et al., 2002].

From this study, we can conclude that making decisions about withholding lifesustaining treatment based on these predictors, is difficult in the first day of life and increasingly difficult thereafter [Meadow et al., 2004]. This is in accordance with the results of Richardson et al. who observed that SNAP scores decrease in predictive power in the second week of life [Meadow et al., 2002]. .

Similarly, Morse et al. found that physicians' predictions get more accurate as the gestational age of the infant increases, but after the infant is born, the prediction accuracy of the physician decreases with time [Mueller et al., 2004]. Therefore, it is vital that physicians receive better information in predicting the outcome especially after the admission day into the NICU. This is where the use of prediction systems in the modern NICU play an important role.

\subsubsection{The Relationship between Time and Contamination-free Prediction}

A study was performed in Greece to evaluate the performance of the SNAP score calculated during the first 12 hours after admission in comparison with the customary first 24 hours. This study was done before the advent of SNAP-II, which incorporated 
the use of data collected within the first 12 hours so that the data would be less 'contaminated' by treatment results. The SNAP score was also taken during the second half of the first day (i.e. the second 12 hours) and was compared with both the first 12 hour score and the 24 hour score in order to observe the effects of time on scoring power.

The group hypothesized that a shorter 12 hour scoring period would be a better predictor for the following reasons:

1. A 12 hour period from admission would be more representative of the status of admission and less contaminated by treatment results

2. A 12 hour period would allow the test to be completed earlier and, therefore, give physicians an earlier reference of inter hospital performance

3. Separating the SNAP scoring period into first and second 12 hour periods could give a better predictive value to the score and give an earlier indicator of the response to treatment.

The group found that SNAP during the second 12 hours alone was a better predictor of both fatality and the length of stay compared to the SNAP score from the first 12 hours and the first 24 hours (including the first 12 hours and the second 12 hours). Also concluded by the group was the fact that the SNAP score of the first 12 hours was as predictive as the SNAP score for the first 24 hours, but not as good as the SNAP score of the second 12 hour period alone. This was an important finding as it implied that if the SNAP score was determined within the first 12 hours it did not lose any of its predictive power [Petridou et al., 1998].

These findings indicate that a score that is more 'contaminated' (i.e. the second 12 hours period) with treatment response is a better indicator of mortality. This is consistent with the findings of Fowlie et al., in which the area under the ROC curve for predicting mortality was greatest using the CRIB score of day 3 and the ultrasound scan of day 3 [Fowlie et al., 1998]. These findings, however, contradict the conclusions of Meadow et al., in which SNAP scores were found to diminish in power as time passes. 


\subsubsection{Temporal Effects on Resource Allocation}

As mentioned earlier, Meadow et al. found that the median length of stay for nonsurvivors in the NICU rose from 5 days in 1991 to 17 in 2001. Meadow et al. asked the following question: were non-surviving infants occupying more NICU beds or survivors? It was determined that non-survivors consistently occupied a very small percentage of NICU beds (never more than 10\%) as compared with adult ICUs where non-survivors accounted for more than $50 \%$ of beds [Meadow et al., 2004].

In accordance with the observation of increased length of life for non-survivors in the NICU, Wilson et al. found that the younger group of infants (the ones who are normally ELBW) are allocated more NICU resources, in particular the mechanical ventilation resources. Ventilation accounts for between 28 and $37 \%$ of the cost of keeping ELBW infants in the NICU, therefore predicting the length of ventilation for infants would aid in distribution of resources and reduction of NICU costs. The most important factor in determining length of ventilation (LOV) was determined to be the gestational age [Wilson et al., 2000].

Therefore, to summarize, the following are key points related to temporal issues in the NICU:

- SNAP scores and clinician prediction are less reliable as time passes [Meadow et al, 2002] and [Mueller et al., 2004]

- The SNAP score taken in the last 12 hours of the admission day is more predictive of mortality and length of stay [Petridou et al., 1998]

- CRIB score at 72 hours combined with ultrasound scan assessment are better predictors of mortality on day 3 than the conventional predictors: birth weight and gestational age [Fowlie et al., 1998]

- After day 3, most infants die due to other complications such as respiratory distress syndrome, intraventricular haemmorage, and pulmonary interstitial emphysema

From the issues discussed in this section, it is clear that a prediction model specially adapted for predicting mortality on day 3 may overcome the current prediction obstacles to solve the treatment and resource allocation problems faced by the NICU. A 
new mortality prediction model is required that moves away from the conventional modeling parameters and techniques to explore new parameters and incorporate the latest NICU trends and technologies.

\subsection{Medical Ideas Research Group}

The Medical Information technology Research Group (MIRG) led by Dr. Frize and Dr. Walker, comprises graduate students from Carleton University, the University of Ottawa and researchers from the Children's Hospital of Eastern Ontario (CHEO) and the Ottawa Hospital. MIRG's goal is to develop information systems integrated with artificial intelligence applications that can aid clinicians in medical decision-making.

There has been much research in the field of missing value imputation and mortality prediction by MIRG researchers in the past. The following is a brief description of research into the temporal effects on prediction by Ye and Walker et al., the development of a hybrid ANN-CBR system for imputation and model creation by Ennett, the development of an ANN research framework tool by Rybchynski and advancements in the development of an on-line real-time NICU prediction system.

\subsubsection{Research in Predictive Power of Day 3 Data}

Winnie Ye of MIRG, performed a study in 2000 to investigate whether an ANN model's ability to estimate the duration of artificial ventilation for neonates increases or decreases as time passes. Data from the Canadian Neonatal Network (CNN) was used. The complete CNN cases for day 1 were 5,118 (from 20,008 total cases) and for day 3 were 2,367 (out of 14,192 total cases).

SNAP was chosen as the scoring system for the project because it was well correlated with mortality and length of stay in NICUs. The six SNAP input variables were categorized instead of simply using their original continuous value. The study attempted to predict whether the need for ventilation was greater or less than 8 hours, 12 hours or 24 hours.

The correct classification rate (CCR) of the ANN was similar to the CP values for the ventilation prediction using categorized data for day 1 and day 3 , but was not consistently higher or lower for either day 1 or day 3 . Therefore, no conclusion on the 
temporal effects on the predictive power could be derived from the results (even though Ye concluded that prediction did not improve as time passed), but the ANN tool's ability to accurately predict ventilation was verified. Significantly higher sensitivities were achieved when the original input data was used instead of the categorized input data. Ye concluded that the "the ANN model does not produce valid predictions for continuousvalued databases". Ye also warned that the input variable set was erroneously selected to include a variable that was not of the SNAP set and therefore, the experiments should be repeated using only variables that are in the SNAP set [Ye, 2000].

Following Ye's study, another MIRG study was performed using the same CNN data. Walker et al. used the ANN tool in predicting ventilation length for day 1 and day 3 data. Mortality predictions were performed but only for day 1 to compare the CCR of the ANN to the CP.

Walker et al. concluded that the CCR value obtained by the ANN was close to or slightly better than the CP for most ventilation outcomes. The results show that the CCR value was slightly higher for day 3 VENT predictions than day 1 predictions, indicating day 3 data gives a slightly more accurate prediction for length of ventilation [Walker et al., 2001].

The tools and methodology used by Ye and Walker et al. had many limitations that have been corrected in this research in order to conduct a more thorough study of temporal effects. The older version of the tool took the highest CCR value and not necessarily the most stable and used its corresponding ASE value, which was not necessarily the smallest error value. Although this research will not look at continuousvalued output, the MIRG tool has since been updated to improve the analysis of continuous-valued output [Rybchynski, 2005].

Another important factor not considered by Ye and Walker et al. was the significant impact of deleting the missing values in the dataset. Deleting the cases with missing values, which are generally the healthier infants, left the dataset skewed with a higher number of sicker babies [Ennett, 2003]. It also reduced the total number of cases used to train the network, causing possible over-generalization by the ANN. This research will correct for this using missing value imputation. 


\subsubsection{Developing a Neonatal Mortality Prediction Model using an ANN-CBR Hybrid}

MIRG combined an ANN and a CBR to impute missing values to better define an ANN model. Two most notable research accomplishments from this research were:

1. Demonstrating the hybrid ANN-CBR system could impute values that were closer to the true missing value than a value found by mean or random imputation.

2. Developing a new neonatal mortality model using the hybrid system that more accurately predicted mortality than other models [Ennett, 2003].

A hybrid ANN and CBR system was proposed by Ennett and Frize, that involved training and testing only the complete cases of a database on an ANN to determine the relative weights of the input variables. Imputation was first done "vertically" to ensure all nine SNAP-II variables were imputed for all cases. Next, "horizontal" expansion was done by imputing missing values in all 30 variables of the dataset. The hybrid tool was successfully used to fill the database of approximately 20,000 cases from a complete set of only 5,000 cases.

Ennett identified a new 13 variable model for predicting neonatal mortality that was different from previous mortality models. The model included all of the SNAPPE-II variables with the exception of the occurrence of multiple seizures (seizure) and the lowest blood pressure (lbloodp). Ennett's model was able to achieve a higher classification performance than the SNAP score models [Ennett, 2003].

\subsubsection{ANN RFW Optimization}

Dawn Rybchynski, a master's student of MIRG, contributed to the group's progress towards their goal of developing artificial intelligence systems for hospitals in several ways. The following are significant contributions of Rybchinski's work to this research:

1. An Artificial Neural Network Research Framework (ANN RFW) that is fully automated allowing the user to quickly optimize the ANN network architecture.

2. A Committee of Classifier Verification Tool (CCVT) to verify the ANN prediction model by showing the case-by-case result.

3. A hybrid of an $\mathrm{ANN}$ and k-nearest neighbour (k-NN) matching case based reasoner (CBR) tool that implemented weight extraction. 
4. Initial steps towards an Outcome Prediction Model Definition Process (OPMDP)

The ANN was updated by Rybchynski as described in section 2.1.3, to determine the optimal neural network architecture through an automated process. Parameters are updated automatically for the ANN and neural network structures with 0 to $2 \mathrm{n}+1$ hidden nodes are automatically attempted by the tool. In addition, 10 completely independent verification sets defined by the user, can be used to verify the ANN model.

The CCVT uses a series of ANN prediction models of different structures to predict the same outcome using a majority rules or voting system. This tool allows verification of a model to ensure it is able to retrain on a new dataset and still perform well.

When using the $\mathrm{kNN}$ tool in the past, MIRG assumed that the solution is linear and extracted ANN weights from a single-layer ANN. Rybchynski simplified the weight extraction process so that the ANN weights of a multi-layered neural network can easily be extracted for the $\mathrm{kNN}$.

Rybchynski developed an OPMDP activity diagram that indicates the sequence of activities that must be performed in outcome prediction. The model acts as an aid in learning how the individual MIRG tools can be integrated into an outcome prediction system [Rybchynski, 2005].

\subsubsection{The Proposed MIRG CDSS System}

Catley of MIRG designed a medical information framework for the integration of various medical applications such as CBRs and ANNs. A future goal of the MIRG team is to link MIRG software tools to the real-time data repository at CHEO, so the data can be processed and analyzed in real-time. An open source medical architecture was designed to support the interchange of medical data and remote, real-time access to it. XML schemas for standardizing the format of medical data were also developed. Work that is currently underway in the construction of a fully integrated web system includes the XML-enabling of the CHEO data repository, integrating fully the CDSS tools with the hospital information systems, implementing wireless alerts for alarming physicians of dramatic changes in a patient's physiological data and many other projects [Catley, $2002]$. 
A step forward in the development of a fully integrated system was the pilot study of the MIRG's ANN tool at CHEO for clinician testing and feedback. A GUI for the tool displays the prediction outcomes of the ANN, as shown in figure 3-1, for three outcomes. An attitude test was performed, to measure the acceptance of the tool by physicians and a performance test was done to compare the tool's predictive power with that of the physician's. The physicians reacted positively to the tool although they commented that it be used as a recommended course of action and not as "raw data" for the parent's of the infant.

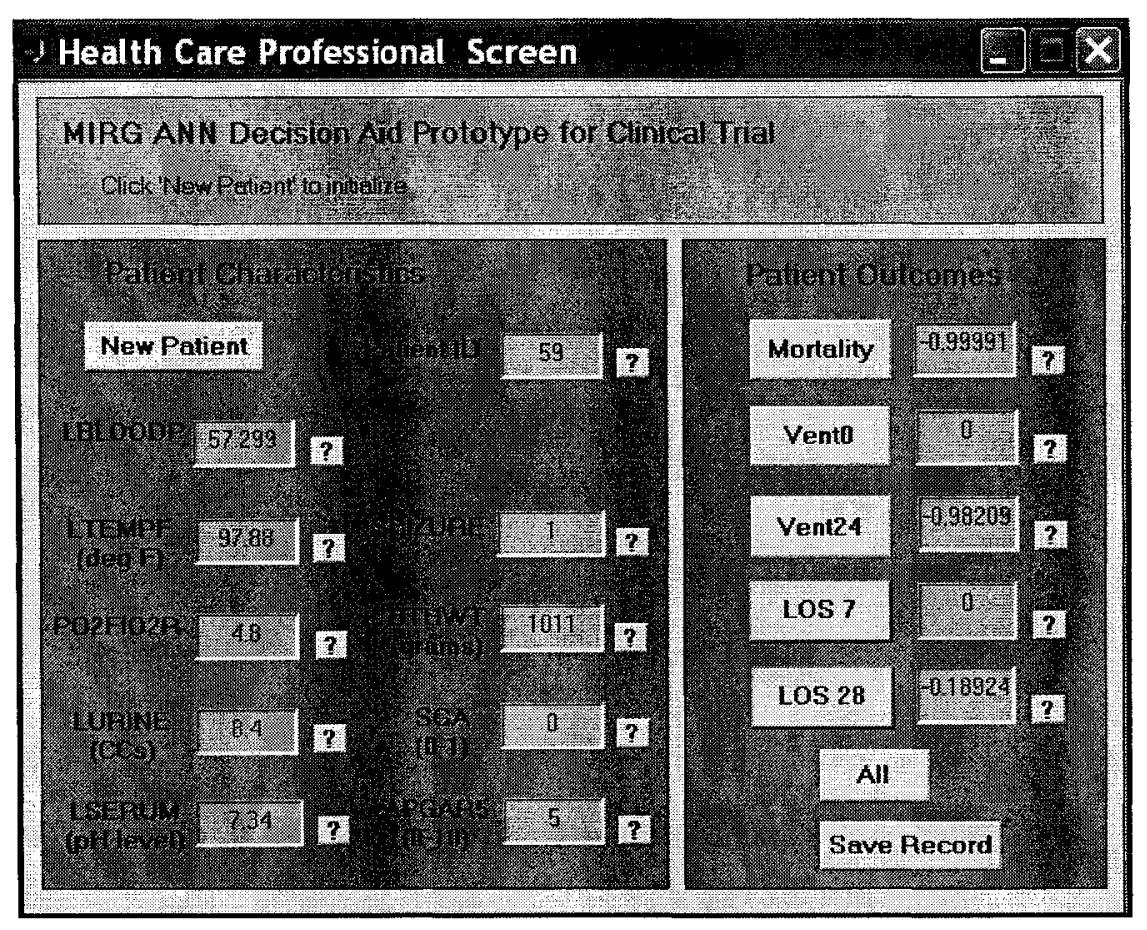

Figure 3-1: GUI for attitude test [Qi, 2005]

In the pilot study, the most significant predictors chosen by physicians were compared to the predictors used by the tool. Gestational age, which the physicians found significant, had not been included in the nine SNAP-II parameters used by the ANN.

The performance of the ANN tool was comparable to the physicians' predictions for the three outcomes when the EPIC database was used. For mortality it was observed that the ANN tool in some instances was able to correctly predict a survivor, when the physician was not able to. The physicians, on the other hand, were able to correctly 
predict non-survivors in some instances that the tool was not able to. Qi uses these results to show that the tool can be used by physician's to increase the accuracy of the prediction of survivors (i.e. the specificity) [Qi, 2005]. As mentioned above, Qi's research also demonstrates that it is important for an ANN tool to have a high sensitivity as well as a high specificity, in order to aid physicians in the clinical environment.

Yet another recent advancement towards a fully integrated prediction system, was the pilot study for the development of a Parent Decision Support tool (PADS) for the NICU. This tool is meant to assist parents in participating in the decision (with the health are team) on whether aggressive therapy should be started, continued or withdrawn based on predictions of mortality, morbidity, likelihood of brain damage and quality of future life. In a pilot and usability study performed to assess whether the basic usability requirements of the tool were fulfilled, positive feedback from clinicians was received. Future work for the tool requires integration of several standalone components such as evidence-based estimation, medical information retrieval and update, real-time physiological conditions update and others. Also, different clinical applications of the tool are to be explored in the future [Yang, 2004; Frize et al., 2005].

A real-time physiological data monitoring and storage system is currently in place at the NICU in CHEO. The first step towards a fully functional real-time prediction system would be the integration of this data monitoring system with the ANN tool. Once this step is complete the ANN can continuously receive the input data it requires to predict an outcome in real-time. The ANN tool must be trained before it is able to output a prediction. The second step required is implementing a training phase in which the ANN trains on previously acquired data. If different prediction models are to be used for data collected on different days, the ANN has to be able to dynamically change the model it is using depending on which day the data was collected.

Therefore, the research into a new mortality model for day 3 data is a vital step towards building a real-time prediction system. However, there are still many gaps in the research that must be addressed before such a system is fully functional. 


\subsection{Methodology}

The following is a brief overview of the methodology of the research. It will be explained in greater detail in the rest of this chapter:

1. The $\mathrm{kNN}$ was used to impute missing values into the day 3 set that had a complete set of SNAPPE-II variables $(\mathrm{N}=2,364)$. Weights from a linear (2layered) network and non-linear (3-layered) network were used in the kNN and the imputed databases were compared to each other and the true values. The results of the $\mathrm{kNN}$ were also compared with Ennett's results for day 1 imputation using the CBR tool.

2. The day 3 database was expanded vertically using the best performing weights from step 1 to get a full set of SNAPPE-II variables for the complete set of day 3 cases $(\mathrm{N}=13,871)$. The performance of this dataset on the ANN was observed.

3. The set of complete SNAPPE-II cases $(\mathrm{N}=2,364)$ was horizontally expanded so that the full set of 28 variables were present.

4. Variables were gradually removed from the full set of 28 variables to find the minimum dataset. Both linear (2-layered) and non-linear (3-layered) networks were used to reduce the variable set.

5. The performance of the minimum dataset found using a linear network ("linear model") was compared to the performance of the minimum dataset found using a non-linear network ("non-linear model"). Both the models were compared to Ennett's day 1 model and the SNAPPE-II model.

\subsection{Artificial Imputation}

Objective 1 of this research was stated as follows in section 1.3:

The ability of the updated case based reasoner tool $(\mathrm{kNN})$ to impute missing values into a dataset will be verified by the imputation of known data values.

In accordance with this objective an artificial dataset was created by deleting known values. The number of variables deleted corresponded to the actual number of missing values for that variable in the entire database so that the artificial database 
mimicked the actual database. The artificial missing values were then imputed using the $\mathrm{kNN}$ case based reasoner, which is the current CBR tool used by MIRG. Ennett had used a CBR tool called the Easy Reasoner, but it was now obsolete and unavailable for use. It is for this reason that the $\mathrm{kNN}$ had to be verified to ensure that it could perform just as well or better than the CBR tool Ennett had used.

Imputation of missing values using the mean of the data or using a random value for the variable was not performed, as these methods were shown to give a poorer performance compared to the imputation using the mean of the closest matching cases [Ennett, 2003]. Ennett had used weights from a linear (2-layered) network only. She did not attempt extracting weights from a non-linear (3-layered) network. So, in the artificial imputation step of this research, the use of linear network weights was compared to the use of non-linear network weights to determine which gave a better imputation as per objective 2 of the thesis:

Missing value imputation using weights extracted from a linear (2layered) neural network is compared to imputation using weights extracted from a non-linear (3-layered) neural network.

\subsubsection{The Artificial Set}

The methodology for step 1 was similar to that used by Ennett and is as follows [Ennett, 2003]:

1. Use only complete cases from the SNAPPE-II variable set for the day 3 database $(\mathrm{N}=2,364)$.

2. Divide database into training set and test set and train ANN to find weights for use in CBR.

3. Extract the ANN weights from the linear (2-layered) network structure and the best performing non-linear (3-layered) network structure and recalibrate such that the largest weight $=100$.

4. Create artificial missing value set by deleting known values.

5. Impute missing values using the linear weights and non-linear weights from the ANN in the $\mathrm{kNN}$. 
6. Compare error between true data and imputed values for the linear and non-linear weights.

7. Use imputed train, test and verification sets on ANN to observe change in performance.

The statistics on the number of missing values in the full day 3 database are shown in table 4-1. The day 3 database initially consisted of a total of 14,261 cases. All values were first checked for outliers. Outliers were either removed or replaced with a valid value as taught by Ennett [Ennett, 2003]. Next, the cases that were missing values for important variables (caselink, mort, bthwt, sga and apgar 5) were removed; after doing so 13,907 cases remained. The cases with ltempf and seizure missing were very few, so they were also removed so 13,871 cases remained. Each of these remaining cases were missing any number from 0 to 4 variables (lbloodp, po2fio2r, lurine, lserum).

Table 4-1: Missing value statistics for each of the SNAPPE-II variables in the day 3 database $(\mathrm{N}=14,261)$ and artificial dataset $(\mathrm{N}=2,364)$

\begin{tabular}{lllll}
\hline Variable & $\begin{array}{l}\text { Valid } \\
\text { data }\end{array}$ & $\begin{array}{l}\text { Cases } \text { with missing } \\
\text { data }\end{array}$ & $\begin{array}{l}\text { Missing } \\
(\%)\end{array}$ & $\begin{array}{l}\text { Missing in artificial } \\
\text { data set (\%) }\end{array}$ \\
\hline Ibloodp & 9462 & 4799 & 33.7 & 34 \\
Itempf & 14227 & 34 & 0.2 & 0 \\
po2fio2r & 3135 & 11126 & 78.0 & 78 \\
lurine & 5730 & 8531 & 59.8 & 60 \\
Iserum & 5347 & 8914 & 62.5 & 62 \\
seizure & 14254 & 7 & 0.0 & 0 \\
bthwt & 14196 & 65 & 0.5 & 0 \\
sga & 14203 & 58 & 0.4 & 0 \\
apgar5 & 14003 & 258 & 1.8 & 0 \\
mort & 14220 & 41 & 2.8 & 0 \\
\hline
\end{tabular}

To create the artificial database, the complete cases of the day 3 database that had a full set of the 9 SNAPPE-II variables were considered (2,364 cases). Values were randomly selected for deletion until the percentage of values missing was equal to the values missing in the actual database for lbloodp, po2fio2r, lurine and lserum as shown in 
the last two columns of table 4-1. The number of missing values missing in the entire database is shown in table 4-2.

Table 4-2: Missing SNAPPE-II value statistics for entire day 3 database $(\mathrm{N}=13,871)$ based on number of missing values

\begin{tabular}{lll}
\hline \multirow{2}{*}{$\begin{array}{c}\text { Number of missing } \\
\text { values }\end{array}$} & \multicolumn{2}{c}{ Entire database, $\boldsymbol{N = 1 3 8 7 1}$} \\
\cline { 2 - 3 } & Frequency & Percent \\
\hline No missing values & 2364 & 17.0 \\
One missing value & 1850 & 13.3 \\
Two missing values & 2356 & 17.0 \\
Three missing values & 3354 & 24.2 \\
Four missing values & 3947 & 28.5 \\
\hline
\end{tabular}

\subsubsection{Artificial Imputation Using Linear and Non-linear Weights}

To obtain weights for the $\mathrm{kNN}$, the complete case set $(\mathrm{N}=2,364)$ was run on the ANN RFW tool to extract the weights of the best performing network. Table 4-3 shows the statistics of the training, testing and verification sets used on the ANN RFW tool. The positive cases are the number of infants that did not survive, while the negative cases are the survivors. The percentage of mortality and survival is also shown in the table.

Table 4-3: Training, test and verification set statistics for complete cases in day 3 database $(\mathrm{N}=2,364)$

\begin{tabular}{lllll}
\hline & $\begin{array}{l}\text { Original } \\
\text { Training Set }\end{array}$ & $\begin{array}{l}\text { Resampled } \\
\text { Training }\end{array}$ & Test Set & Verification Set \\
\hline Positive Cases & 103 & 232 & 47 & 71 \\
Negative Cases & 929 & 929 & 501 & 713 \\
Total & 1032 & 1161 & 548 & 784 \\
Positive \% & 10 & 20 & 8.6 & 9.1 \\
Negative \% & 90 & 80 & 91.4 & 90.9 \\
\hline
\end{tabular}

Weights were extracted from both the linear ANN structure (with 0 hidden nodes) and the best performing non-linear structure that was found using the selection criterion explained in section 2.2.5. This method is shown in figure 4-1. 
In the initial ANN RFW test, resampling was not performed because the mortality rate of the complete case dataset was close to $10 \%$, which is fairly high compared to the mortality rates of other MIRG datasets (3-4\%) that had required resampling in the past [Ennett et al., 2001]. When resampling was attempted so that the mortality rate was $20 \%$ (column 2 of table 4-3), the sensitivity of the ANN was able to increase greatly and so resampling to $20 \%$ was used in all subsequent ANN experiments.

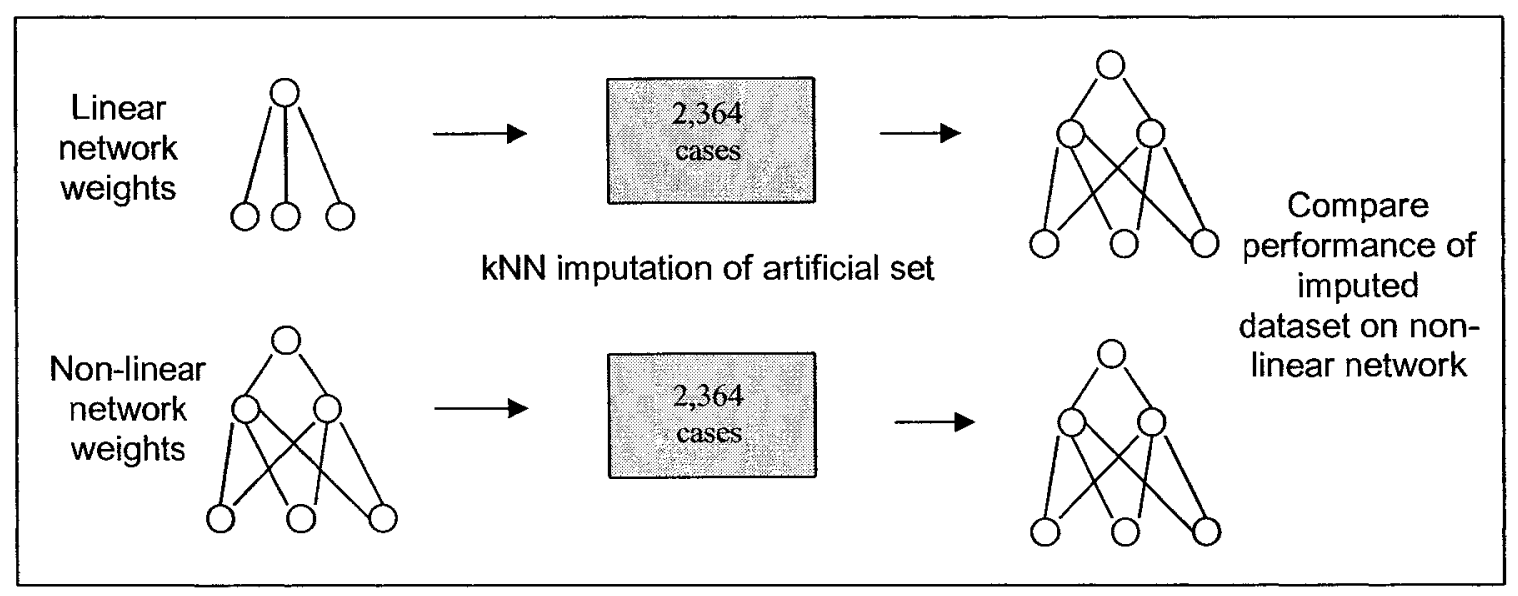

Figure 4-1: Methodology of kNN verification

After the artificial set was imputed using both the linear and non-linear network weights the error for each was compared to the true values of the set. The ANN performance of the artificially imputed set was also compared to the original's performance by running the imputed datasets on a non-linear network structure as shown in figure 4-1. Ennett had run the imputed test and verification sets with the original (nonimputed) training set to determine how close the imputed values were to the original. But, because the later steps of this research require imputed data to be used in all sets (training, test and verification) all three imputed sets were run on the ANN tool.

The artificial imputation step was a crucial step in this research because it determined which type of network weights - linear or non-linear network weights would be used in all subsequent imputation (vertical and horizontal) steps.

\subsection{Vertical Expansion}

The third objective stated in section 1.3 of this research is as follows: 
The kNN will then be used to impute missing values to "vertically" expand the day 3 dataset, so a greater number of cases are used to more effectively train the ANN

Vertical expansion was a term coined by Ennett to mean the imputation of missing values into all cases in the dataset missing values for a specific set of variables, in this case the 9 SNAPPE-II variables. The vertical expansion steps, initially created by Ennett and adapted to suit the day 3 model, are as follows [Ennett, 2003]:

1. Using ANN weights that gave the best imputation results from preliminary complete case experiments, impute unknown missing values using the $\mathrm{kNN}$ and the complete cases in match set $(\mathrm{N}=2,364)$. Start with cases missing only one SNAPPE-II value, then two, three and four (no missing values for mortality, bthwt, sga, apgar 5 , seizure or ltempf).

2. Check statistics of imputed database to show that means are less influenced by the sick babies.

3. Randomly separate complete and imputed databases $(N=13,871)$ into training, test and verification sets for use in ANN.

4. Attempt ANN structures with 0 to $19(2 n+1)$ hidden nodes and determine which performed the best.

Ennett had compared the mean and random imputation methods to the CBR imputation and proved that imputation using the CBR resulted in the best ANN performance. Therefore, the mean and random imputations were not attempted in this research. Ennett had only used a linear (2-layered) ANN for her research. This research attempts both linear and non-linear structures with 0 to $2 n+1$ hidden nodes (where $n$ is the number of inputs) to find the best-performing structure.

The statistics of the entire database are shown in table 4-4. The complete case set $(2,364)$ used in step 1 is used again as the initial match set. The $\mathrm{kNN}$ is able to impute 
the rest of the missing values in database using this match set. There are a maximum of four missing values for any given case (lbloodp, po2fio2r, lurine, lserum).

As explained in 2.4.1, Ennett discovered that infants with a complete set of variables were the most sick infants in the database [Ennett et al., 2001]. The mortality rate for the complete set of day 3 cases was $10 \%$. The mortality rate for the entire set of 13,871 cases was $2.3 \%$. Therefore, a mortality model built on the complete cases would not be applicable to the entire database of infants. Therefore, vertical expansion according to Ennett, was meant to broaden the spectrum of infants by including those that were not as severely ill.

To vertically expand the database, another problem had to be solved. If the complete case set and the sickest infants were to be used as the match set for the rest of the database, how could one ensure that the imputed values of the less sick infants were not drastically biased by the sick match set? Ennett overcame this by imputing values into the set missing only one value and therefore, were most close to the infants missing no values. Once this set of cases had been imputed, they were added to the match base to gradually influence the mean of the match base. Next, the cases missing two variables were imputed and so on until the set with four missing variables had been imputed using a match case of all previously imputed and complete cases [Ennett, 2003].

Using the mean imputation technique ensures that the imputed values are not out-ofrange or 'unreal' because it is performed on accurate variable values. The CNN database is checked for reliability to ensure that the values it contains are accurate [Frize, 2003]. In the previous step an artificial set was created by removing values and then these values were replaced using the $\mathrm{kNN}$ and the mean imputation method. The results of this step proved that the $\mathrm{kNN}$ can impute values that are fairly close to the true values. So, the imputation technique was verified for the vertical expansion step. Similar to the artificial set, the vertically expanded set will have imputed values that are close to the true values that were missing. 


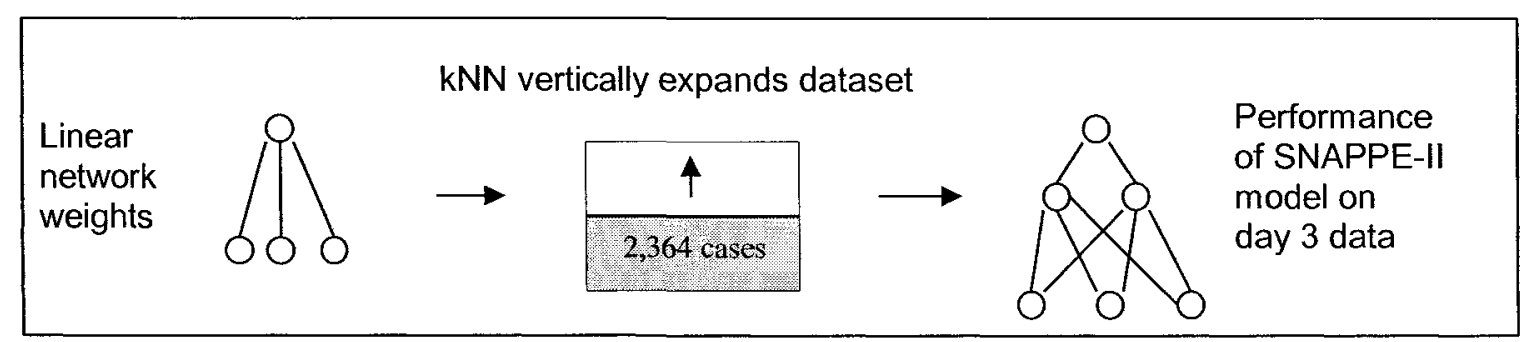

Figure 4-2: Methodology of vertical expansion

After vertical expansion the entire database of 13,871 cases had values for all 9 SNAPPE-II variables. This is shown in figure 4-2. The linear network weights are used for the $\mathrm{kNN}$ in this step because these are the weights that are chosen in the $\mathrm{kNN}$ verification step. After expansion, the mortality rate of the vertically imputed set was quite low $(2.3 \%)$. However, resampling was performed to ensure an acceptable sensitivity was achieved. The resampled dataset was run on the ANN RFW and its results were analyzed.

Table 4-4: Training, Test and verification set statistics for entire day 3 database after vertical imputation $(\mathrm{N}=13,871)$

\begin{tabular}{lllll}
\hline & $\begin{array}{l}\text { Original } \\
\text { Training Set }\end{array}$ & $\begin{array}{l}\text { Resampled } \\
\text { Training }\end{array}$ & Test Set & Verification Set \\
\hline Positive Cases & 167 & 1495 & 2985 & 100 \\
Negative Cases & 5981 & 5981 & 87 & 4552 \\
Total & 6148 & 7476 & 3071 & 4652 \\
Positive \% & 2.7 & 20 & 2.8 & 2.1 \\
Negative \% & 97.3 & 80 & 97.2 & 97.9 \\
\hline
\end{tabular}

\subsection{Horizontal Expansion}

The fourth objective as outlined in section 1.3 was as follows:

The kNN will be used to impute missing variables to "horizontally"
expand the day 3 dataset, so a greater number of variables are considered
in the creation of the mortality prediction model for day 3 data.

Horizontal expansion was a term coined by Ennett to mean the imputation of additional variables in a dataset to increase the number of variables. Horizontal 
expansion differs from vertical expansion in that a variable that was not a part of the dataset initially is added with an imputed value, whereas vertical expansion simply imputes the missing value into a variable that already existed in a dataset. The steps for horizontal expansion are shown below along with the steps for finding the minimum dataset and comparing the models:

\section{Horizontal Expansion}

1. Separate SNAPPE-II database into data sets with complete cases for an increasing number of variables.

2. Divide database into training, test and verification sets, and train ANN to find weights for $\mathrm{kNN}$ - extract the weights.

3. Use all complete cases in $\mathrm{kNN}$ match set (query set is remaining cases from 2,364 cases) and impute missing values starting with cases missing one value, then two, three, etc.

Finding the Minimum Dataset

4. Randomly separate complete and imputed databases into training, test and verification sets for ANN RFW.

5. Run ANN to eliminate variables, then re-run $\mathrm{ANN}$ to observe impact on classification performance.

6. When classification performance (i.e. sensitivity) begins to degrade, extract final set of $\mathrm{ANN}$ weights for use as $\mathrm{kNN}$ weights.

7. Impute missing values in remainder of database using only the refined variable set starting with cases missing one value, then two, three, etc.

8. Separate complete/imputed database into training, test and verification sets $(N=13871)$.

Comparing the Models

9. Run ANN RFW and observe classification performance.

10. Compare minimum dataset variables and performance to other prediction models. 


\subsubsection{Variable Set Selection}

As per step 1 in the horizontal expansion methodology given above, the data was split according to the percentage of missing values in the database. The variables and the percentage missing within the full database and within the SNAPPE-II dataset are shown in table 4-5 in increasing order. The cases that are not missing any variables from the set are used for ANN training and weight extraction. The number in column 5 of table 4-5 indicates how many cases are used on the ANN for each of the three sets.

As the number of variables increases, the number of cases with no missing variables decreases. With too few cases the ANN risks being undertrained. Masters states there should be at least 10 times the cases as there are variables in the dataset [Masters, 1993]. After 28 variables the cases decreased to almost less than 10 times the number of variables. Therefore, 28 was the maximum number of variables that were kept in the set. The 28 variables are grouped into three, so that variables with a similar number of cases are together.

As stated above in section 2.4.1, as the number of missing values decreases, the mortality rate or sickness of a group increases. This is apparent from the mortality rate of set C. This group has the highest mortality rate (13.2\%) because it has a complete set of 28 variables. Set B has the next highest and then set A. Imputation of the variables in this set is done gradually to ensure that the imputed values are not biased by the sickest infants of the group.

Table 4-5: Missing value statistics for all 40 variables in day 3 database $(\mathrm{N}=14,261)$

\begin{tabular}{llllll}
\hline & & $\begin{array}{l}\text { Missing } \\
(\%)\end{array}$ & $\begin{array}{l}\text { Complete } \\
\text { Cases in } \\
\text { full set }\end{array}$ & $\begin{array}{l}\text { Cases in } \\
\text { snappe-2 set }\end{array}$ & $\begin{array}{l}\text { Number and \% of } \\
\text { MORT }\end{array}$ \\
\hline seizure & 1 & 0 & 14254 & 2364 \\
Itempf & 2 & 0 & 14227 & 2364 \\
sga & 3 & 0 & 14203 & 2364 \\
bthweight & 4 & 0 & 14196 & 2364 \\
apgar5 & 5 & 0 & 14003 & 2364 \\
Ibloodp & 6 & 0 & 9462 & 2364 \\
lurine & 7 & 0 & 5730 & 2364 & \\
Iserum & 8 & 0 & 5347 & 2364 & \\
\hline
\end{tabular}




\begin{tabular}{llllll}
\hline po2fio2r & 9 & 0 & 3135 & 2364 & \\
A & & & & & \\
apnea & 10 & 0 & 14251 & 2364 & \\
guaiac & 11 & 0 & 14252 & 2364 & \\
hheartr & 12 & 0 & 14239 & 2364 & \\
Iheartr & 13 & 0 & 14206 & 2364 & \\
hbloodp & 14 & 0 & 10090 & 2363 & \\
Ipo2po2 & 15 & 0.1 & 3199 & 2360 & \\
hpco2 & 16 & 0.4 & 5290 & 2350 & \\
hrespr & 17 & 1.1 & 14203 & 2324 & $\mathrm{~N}=163 ; 9.6 \%$ \\
hsodium & 18 & 6.5 & 7460 & 2175 & \\
hgluc & 19 & 8.1 & 8518 & 2021 & \\
lgluc & 20 & 13 & 7469 & 1921 & \\
hpotass & 21 & 13.7 & 6360 & 1792 & \\
Isodium & 22 & 14.5 & 6209 & 1715 & \\
Ipotass & 23 & 21.4 & 5471 & 1700 & \\
\hline B & & & & & \\
hidbili & 24 & 21.8 & 8587 & 1390 & \\
oi & 25 & 24.2 & 2365 & 1077 & \\
\hline C & & & & & \\
hhema & 26 & 46.5 & 3660 & 622 & \\
Iwbc & 27 & 48.1 & 3572 & 583 & \\
Iplt & 28 & 49.2 & 3422 & 552 & \\
\hline D & & & & & \\
hdbili & 29 & 50.8 & 5694 & 307 & \\
Ihema & 30 & 51.8 & 3173 & 288 & \\
hitnrat & 31 & 58.8 & 2664 & 214 & \\
lanc & 32 & 58.8 & 2663 & 214 & \\
hcreat & 33 & 59.5 & 2127 & 155 & \\
\hline E & & & & & \\
hcalcion & 40 & 80.2 & 1139 & 0 & \\
hbun & 35 & 67.6 & 1778 & 66 & \\
Icalctot & 36 & 72 & 1704 & 66 & \\
hhco3 & 37 & 76.3 & 1405 & 43 & \\
Ihco3 & 38 & 76.8 & 1306 & 43 & \\
\hline hcalcion & 39 & 78.8 & 1290 & 0 & \\
\hline
\end{tabular}

The number of missing values for each variable of the day 3 database is different from the numbers in the day 1 database used by Ennett. Therefore, the three variable sets 
chosen for day 3 imputation differ from the variable sets chosen by Ennett for the day 1 database. The three variable sets used in the expansion of the day 3 database were as follows:

- Set A (23 input variables, 1700 cases, mortality rate $=9.6 \%$ );

- Set B (25 input variables, 1077 cases, mortality rate = 10.2\%); and

- $\quad$ Set $\mathrm{C}$ ( 28 input variables, 552 cases, mortality rate $=13.2 \%$ ).

As per step 2 in the horizontal expansion methodology stated above, the three sets were divided into training, test and verification sets and were run on the ANN RFW. The statistics of these sets are shown in tables 4-6 to 4-9 for sets A to C. Weights for each set were extracted from a linear (2-layered) structure. Linear network weights were taken over non-linear network weights because they were found to perform better in the $\mathrm{kNN}$ imputation of the artificial set as will be explained in detail in the results section.

Table 4-6: Training, test and verification sets for set $\mathrm{A}$ of the horizontal imputation $(\mathrm{N}=1,700)$

\begin{tabular}{lllll}
\hline & $\begin{array}{l}\text { Original } \\
\text { Training Set }\end{array}$ & $\begin{array}{l}\text { Resampled } \\
\text { Training }\end{array}$ & Test Set & Verification Set \\
\hline Positive Cases & 75 & 167 & 38 & 50 \\
Negative Cases & 667 & 667 & 363 & 507 \\
Total & 742 & 834 & 401 & 557 \\
Positive \% & 10.1 & 20 & 9.5 & 9 \\
Negative \% & 89.9 & 80 & 90.5 & 91 \\
\hline
\end{tabular}

Table 4-7: Training, test and verification sets for set $\mathrm{B}$ of the horizontal imputation $(\mathrm{N}=1,077)$

\begin{tabular}{lllll}
\hline & $\begin{array}{l}\text { Original } \\
\text { Training Set }\end{array}$ & $\begin{array}{l}\text { Resampled } \\
\text { Training }\end{array}$ & Test Set & Verification Set \\
\hline Positive Cases & 47 & 113 & 29 & 100 \\
Negative Cases & 453 & 453 & 190 & 452 \\
Total & 500 & 566 & 219 & 462 \\
Positive \% & 9.4 & 20 & 13.2 & 2.1 \\
Negative \% & 90.6 & 80 & 86.8 & 97.9 \\
\hline
\end{tabular}


Table 4-8: Training, test and verification sets for set $\mathrm{C}$ of the horizontal imputation $(\mathrm{N}=552)$

\begin{tabular}{lllll}
\hline & $\begin{array}{l}\text { Original } \\
\text { Training Set }\end{array}$ & $\begin{array}{l}\text { Resampled } \\
\text { Training }\end{array}$ & Test Set & Verification Set \\
\hline Positive Cases & 33 & 53 & 14 & 26 \\
Negative Cases & 212 & 212 & 102 & 165 \\
Total & 245 & 265 & 116 & 191 \\
Positive \% & 13.5 & 20 & 12.1 & 86.4 \\
Negative \% & 86.5 & 80 & 87.9 & 13.6 \\
\hline
\end{tabular}

\subsubsection{Types of Horizontal expansion}

Ennett proposed two types of horizontal expansion methods. The first is variablewise horizontal expansion and the second is step-wise horizontal expansion.

1. Variable-wise horizontal expansion comprises first imputing the largest number of variables. In this case it would be the 28 variables of set $\mathrm{C}$. Then the missing values of set $B$ would be imputed using the weights of set $C$. The question arises as to which weights are used for set $\mathrm{A}$ - the weights of set $\mathrm{B}$ or set $C$ ? Another issue with this method is that there are not enough cases for set $\mathrm{C}$ for the initial imputation.

2. Step-wise horizontal expansion comprises imputing the missing variables of set $\mathrm{A}$. Then the new variables of set $\mathrm{B}$ that are not included in set $\mathrm{A}$ are imputed and the same is done for set $\mathrm{C}$. This was the method chosen by Ennett to use because the match set was enriched to gradually change the mean values and a larger dataset for set $\mathrm{A}$ would be available and therefore would make a good match set for the succeeding imputations. The drawback of this method is that three separate weight sets must be used because for each variable set the order of importance may change. This was also the method chosen for this research. A visual of the method is shown in figure 4-1.

Figure 4-1 shows the 1700 cases that are not missing variables for the 23 variables included in set $\mathrm{A}$ are used as the match set to impute the 664 cases missing variables for set $A$. In the next step, the 1,077 cases that are complete for 
all 25 variables are used as the match set to impute all 25 variables into the remaining 1,287 cases. The 1,287 cases already have a complete set of 23 variables, so only cases missing the additional 2 variables have to be imputed. The step is repeated for the additional 3 variables added in set $\mathrm{C}$. The result is a set of 2,364 cases with a full set of 28 variables.

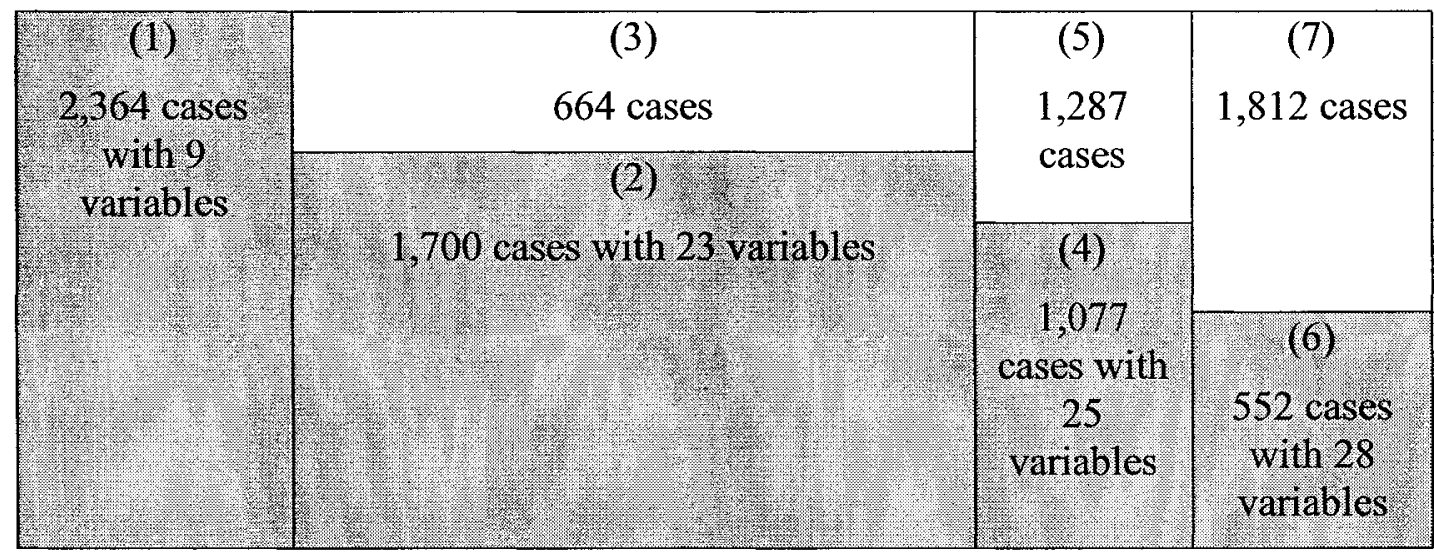

Figure 4-3: Step-wise horizontal imputation of day 3 database for 28 variables $(\mathrm{N}=2364)$

The number of missing values for each dataset are shown in tables 4-9 to 4-12. As explained in section 3.2, the values were imputed gradually so that the dataset for set A that is not missing any values was used as the initial match set to impute cases missing one value and therefore, similar in sickness to the complete set. These were then added to the match set and used to impute cases missing two values, and so on until cases missing seven values were imputed and added to the match set. The same was done for set $B$, which could have a maximum of two missing values and set $C$, which could have a maximum of three missing values as shown in figure 4-1 above. 
Table 4-9: Missing value statistics for entire day 3 database

by number of missing values $(\mathrm{N}=13,871)$

\begin{tabular}{lll}
\hline \multirow{2}{*}{$\begin{array}{l}\text { Number of } \text { values } \\
\text { No missing values }\end{array}$} & \multicolumn{2}{l}{ Entire database, $\boldsymbol{N = 1 3 8 7 1}$} \\
\cline { 2 - 3 } & Frequency & Percent \\
\hline One missing value & 2364 & 17.0 \\
Two missing values & 2356 & 13.3 \\
Three missing values & 3354 & 17.0 \\
Four missing values & 3947 & 24.2 \\
\hline
\end{tabular}

Table 4-10: Missing value statistics for cases missing values for the set $A$ variables $(\mathrm{N}=2,364)$

\begin{tabular}{lll}
\hline \multirow{2}{*}{$\begin{array}{l}\text { Number of } \text { values } \\
\text { Zero missing value }\end{array}$} & \multicolumn{2}{l}{ Entire database, $\mathbf{N = 2 3 6 4}$} \\
\cline { 2 - 3 } One missing value & 1700 & 71.9 \\
Two missing values & 297 & 2.6 \\
Three missing values & 100 & 12.6 \\
Four missing values & 156 & 4.2 \\
Five missing values & 16 & 6.6 \\
Six missing values & 32 & 0.7 \\
Seven missing values & 2 & 1.4 \\
\hline
\end{tabular}

Table 4-11: Missing value statistics for cases missing values for set $B$ variables $(N=2,364)$

\begin{tabular}{lll}
\hline \multirow{2}{*}{$\begin{array}{l}\text { Number of missing } \\
\text { values }\end{array}$} & \multicolumn{2}{l}{ Entire database, $\boldsymbol{N = 2 3 6 4}$} \\
\cline { 2 - 3 } & Frequency & Percent \\
\hline Zero missing value & 1368 & 57.9 \\
One missing values & 904 & 38.2 \\
Two missing values & 92 & 3.9 \\
\hline
\end{tabular}

Table 4-12: Missing value statistics for cases missing values for set $\mathrm{C}$ variables $(\mathrm{N}=2,364)$

\begin{tabular}{lll}
\hline \multirow{2}{*}{$\begin{array}{l}\text { Number of } \text { values } \\
\text { vero missing value }\end{array}$} & \multicolumn{2}{l}{ Entire database, $\boldsymbol{N = 2 3 6 4}$} \\
\cline { 2 - 3 } & Frequency & Percent \\
\hline Zere missing value & 76 & 49.1 \\
Two missing values & 57 & 3.2 \\
Three missing values & 1070 & 2.4 \\
\hline
\end{tabular}




\subsection{Finding the Minimum Dataset}

The steps 4 to 8 in the method steps explained in section 3.3 above explain in steps how the minimum dataset ('model') is found.

\subsubsection{Variable Reduction using Linear Network and Non-linear Network Structures}

After the horizontal expansion step was complete, a full database consisting of 28 variables for 2,364 cases had been imputed. The goal of this research is to find a neonatal mortality model that gives a better performance than the SNAPPE-II model. The SNAPPE-II mortality model is currently used to predict mortality after admission day, even though it was developed for admission day prediction and using admission day data [Richardson et al., 1998]. It, and the 13 variable MIRG model developed by Ennett for admission day prediction were developed using logistic regression and a linear neural network structure respectively. Non-linear neural network structures were not used in the development of these models, and therefore, non-linear relationships that may have existed in the medical data upon which they were developed, were not discovered.

Table 4-13: Training, test and verification sets for horizontally imputed database $(\mathrm{N}=2,364)$

\begin{tabular}{lllll}
\hline & $\begin{array}{l}\text { Original } \\
\text { Training Set }\end{array}$ & $\begin{array}{l}\text { Resampled } \\
\text { Training }\end{array}$ & Test Set & Verification Set \\
\hline Positive Cases & 89 & 235 & 62 & 70 \\
Negative Cases & 941 & 941 & 491 & 711 \\
Total & 1030 & 1176 & 553 & 781 \\
Positive \% & 8.6 & 20 & 11.2 & 9 \\
Negative \% & 91.4 & 80 & 88.8 & 91 \\
\hline
\end{tabular}

Given the expanded 28 variable dataset of day 3 data, the next step was to run the data on the ANN RFW to find the variable weight order. The statistics of the training, test and verification sets are shown in table 4-13. Using weight decay and weight elimination as explained in section 2.1.2, the least important weights and therefore the smallest weights, are gradually removed until the network performance degrades. The 
results obtained from dataset reduction are shown in appendix $\mathrm{C}$. At this point, the minimum dataset and the relative importance of each variable in the dataset are found. The horizontal expansion step and variable reduction step are shown below in figure 4-4.

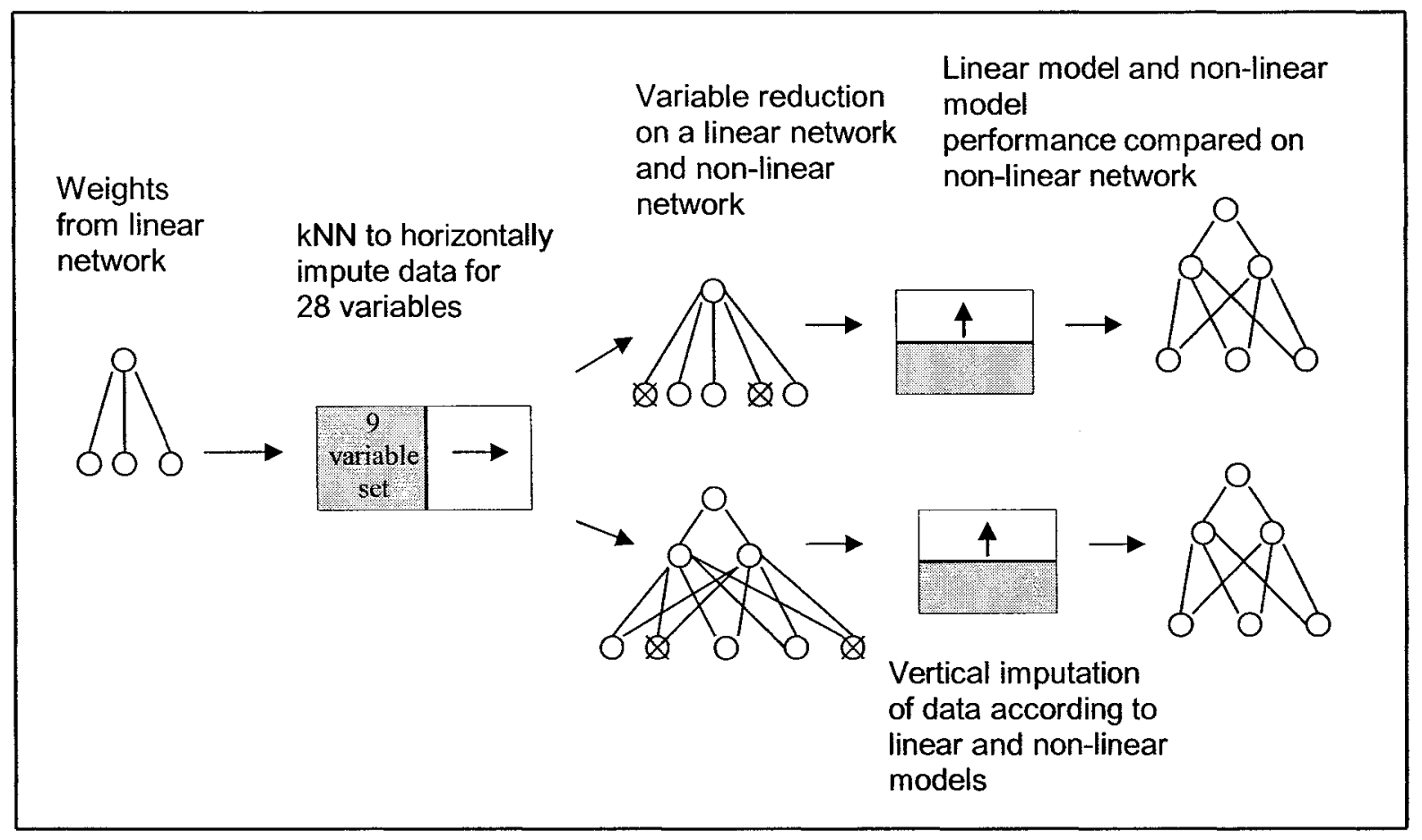

Figure 4-4: Methodology of horizontal expansion, variable reduction and model comparison

\subsection{Comparing the Models}

Objective 5 in section 1.3 was as follows:

Compare the minimum data set found using a linear network with the minimum data set found using a non-linear network

The weights of both minimum datasets - the one found on a linear network structure ('linear model') and the one found on a non-linear network structure ('nonlinear model') - are then used in the $\mathrm{kNN}$ to impute the full 13,871 cases, but only using the variables that exist in the minimum dataset. This step is shown in figure 4-4. The complete set of cases are then run on the ANN RFW again to observe and compare their mortality prediction performance. The statistics of the linear model set and non-linear 
model set are shown in tables 4-14 and 4-15. A day 3 data set containing Ennett's 13 variable model developed on day 1 data, is also imputed for comparison with the linear and non-linear models developed on day 3 data. The statistics of this set is shown in table 4-16.

Table 4-14: Training, test and verification sets for imputed database for 13 variable linear model $(\mathrm{N}=13,871)$

\begin{tabular}{lllll}
\hline & $\begin{array}{l}\text { Original } \\
\text { Training Set }\end{array}$ & $\begin{array}{l}\text { Resampled } \\
\text { Training }\end{array}$ & Test Set & Verification Set \\
\hline Positive Cases & 156 & 1498 & 83 & 114 \\
Negative Cases & 5992 & 5992 & 2988 & 4538 \\
Total & 6148 & 7490 & 3071 & 4652 \\
Positive \% & 2.5 & 20 & 2.7 & 2.5 \\
Negative \% & 97.5 & 80 & 97.3 & 97.5 \\
\hline
\end{tabular}

Table 4-15: Training, test and verification sets for imputed database for 13 variable non-linear model $(\mathrm{N}=13,871)$

\begin{tabular}{lllll}
\hline & $\begin{array}{l}\text { Original } \\
\text { Training Set }\end{array}$ & $\begin{array}{l}\text { Resampled } \\
\text { Training }\end{array}$ & Test Set & Verification Set \\
\hline Positive Cases & 156 & 1498 & 83 & 114 \\
Negative Cases & 5992 & 5992 & 2988 & 4538 \\
Total & 6148 & 7490 & 3071 & 4652 \\
Positive \% & 2.5 & 20 & 2.7 & 2.5 \\
Negative \% & 97.5 & 80 & 97.3 & 97.5 \\
\hline
\end{tabular}

Table 4-16: Training, test and verification sets for imputed database for 13 variable Ennett model $(\mathrm{N}=13,871)$

\begin{tabular}{lllll}
\hline & $\begin{array}{l}\text { Original } \\
\text { Training Set }\end{array}$ & $\begin{array}{l}\text { Resampled } \\
\text { Training }\end{array}$ & Test Set & Verification Set \\
\hline Positive Cases & 156 & 1498 & 83 & 114 \\
Negative Cases & 5992 & 5992 & 2099 & 4538 \\
Total & 6148 & 7490 & 3071 & 4652 \\
Positive \% & 2.5 & 20 & 2.7 & 2.5 \\
Negative \% & 97.5 & 80 & 97.3 & 97.5 \\
\hline
\end{tabular}


Objective 6 of the thesis as outline in section 1.3 is as follows:

Find the 3-layered neural network structure of the $2 n+1$ structures (where $\mathrm{n}$ is the number of input variables to the network) that gives the best performance on day 3 data

The best performing structures of the $2 n+1$ structures that are attempted by the ANN RFW tool are recorded and analyzed. It is hypothesized that the models built using only linear structures such as the linear model found for day 3, Ennett's model found for day 1 and the SNAPPE-II variable model found using logistic regression, will perform better on a linear structure ( 0 hidden nodes). It is also expected that the non-linear model found for day 3 data will perform better on 3-layered network structures and will give an overall better performance (i.e. higher sensitivity) because it is able to find the non-linear relationships between the variables, that the linear models are not able to find. 


\subsection{Results}

\subsection{Imputation of Artificial Set}

\subsubsection{Original Dataset}

The complete cases of the day 3 database $(2,364$ cases in total) with a mortality rate of $9.7 \%$ were run on the ANN RFW to predict mortality. The three best performing structures are shown in table 5-1. The best performance recorded was on a structure with 10 hidden nodes, in which the average sensitivity over the 10 verification sets was $22.4 \%$ and the specificity was $96.1 \%$. The structures with 4 and 6 hidden nodes achieved an even lower average sensitivity. As stated in section 2.1.2 it is desired to achieve a sensitivity of at least $50 \%$. These results are significantly lower than the desired sensitivity, therefore resampling the data was attempted.

Table 5-1: ANN Performance of original day 3 dataset $(\mathrm{N}=2,364)$ for predicting mortality

\begin{tabular}{|l|l|l|l|l|}
\hline \multicolumn{2}{|l}{} & \multicolumn{3}{l}{ 4 hidden } \\
\hline \multicolumn{2}{|l|}{ Best test epoch } & 1750 & 1999 & 1359 \\
\hline \multirow{2}{*}{ Log sensitivity } & Best test & 0.037165 & 0.057323 & 0.058214 \\
\cline { 2 - 5 } & Verification average & 0.020051 & 0.021984 & $\mathbf{0 . 0 2 4 2 7 4}$ \\
\hline \multirow{2}{*}{ Sensitivity (\%) } & Best test & 27.7 & 34.0 & 34.0 \\
\cline { 2 - 5 } & Verification average & 20.4 & 21.2 & $\mathbf{2 2 . 4}$ \\
\hline \multirow{2}{*}{ Specificity (\%) } & Best test & 96.2 & 94.4 & 95.6 \\
\cline { 2 - 5 } & Verification average & 97.0 & 95.9 & 96.1 \\
\hline Classification Rate (CR) (\%) & 90.3 & 89.2 & 90.3 \\
\hline Area under ROC & 71.0 & 67.5 & 68.7 \\
\hline
\end{tabular}

\subsubsection{Resampled Dataset}

Resampling was attempted in order to increase the mortality rate from $9.7 \%$ to $20 \%$. Table 5-2 shows the results of the three best performing structures when the ANN RFW was run using the resampled set to predict mortality. 
Table 5-2: ANN Performance results of resampled day 3 dataset for predicting mortality $(\mathrm{N}=2,364)$

\begin{tabular}{|l|l|l|l|l|}
\hline \multicolumn{2}{|l}{4 hidden } & 9 hidden & 18 hidden \\
\hline \multicolumn{2}{|l|}{ Best test epoch } & 1012 & 1307 & 348 \\
\hline \multirow{2}{*}{ Log sensitivity } & Best test & 0.11353 & 0.10276 & 0.091306 \\
\cline { 2 - 5 } & Verification average & $\mathbf{0 . 0 6 7 4 2 8}$ & 0.032595 & 0.062364 \\
\hline \multirow{2}{*}{ Sensitivity (\%) } & Best test & 46.8 & 44.7 & 42.6 \\
\cline { 2 - 5 } & Verification average & 37.0 & 26.6 & 36.2 \\
\hline \multirow{2}{*}{ Specificity (\%) } & Best test & 91.4 & 92.0 & 91.6 \\
\cline { 2 - 5 } & Verification average & 90.5 & 91.3 & 91.5 \\
\hline Classification Rate (CR) (\%) & 87.6 & 88.0 & 87.4 \\
\hline Area under ROC & 71.8 & 69.1 & 71.9 \\
\hline
\end{tabular}

As expected (see section 2.2.2.1) resampling increased the sensitivity of the ANN. An average sensitivity of $37 \%$ was achieved using a non-linear network with 4 hidden nodes. The ROC for the resampled set improved for the best performing structure (from $68.7 \%$ to $71.8 \%$ ), but the classification rate fell from $90.3 \%$ to $87.6 \%$ as did the specificity from $95.6 \%$ to $91.4 \%$. This was most likely because the resampled set consists of a greater number of mortality cases $(20 \%)$ compared to the original set (9.7\%). So, the network improves its ability to predict which infant will die (the true positives) and is able to increase its sensitivity. But the network does this at the cost of the specificity since it sees a smaller ratio of survivors in the resampled set $(80 \%)$ compared to the original set $(90.3 \%)$.

Since the target is to obtain a sensitivity of greater than $50 \%$ while maintaining a specificity of at least $90 \%$, this lower specificity is still acceptable given that the sensitivity has increased considerably. Because of the improved sensitivity, resampling will be performed in all subsequent tests in order to maximize the sensitivity.

The weights from the structure with four hidden nodes were extracted using the extended Garson Goh method developed by Rybchynski as explained in section 2.2.6. The weights are shown in table 5-3 after they were normalized from 1 to 100 so they could be used in the $\mathrm{kNN}$. 
Table 5-3: Variable weights extracted from the non-linear network with four hidden nodes $($ wnot $=0.4996)$

\begin{tabular}{ll}
\hline Variables & Normalized Weight \\
\hline po2fio2r & 100 \\
Iserum & 99 \\
bthwt & 98 \\
Ibloodp & 89 \\
sga & 87 \\
lurine & 66 \\
apgar5 & 53 \\
seizure & 46 \\
Itempf & 27 \\
\hline
\end{tabular}

\subsection{3 kNN Imputation using Non-Linear weights}

Imputation was performed using the $\mathrm{kNN}$ tool and the non-linear weights that are shown in table 5-3. Table 5-4 shows $\mathrm{p}$ values from $\mathrm{t}$-tests of the $\mathrm{kNN}$ imputation for each missing variable.

Table 5-4: A comparison of the $P$ values from day 3 imputation using the $\mathrm{kNN}$ compared to day 1 imputation using the CBR

\begin{tabular}{|l|l|l|l|l|}
\hline & Ibloodp & po2fio2r & lurine & Iserum \\
\hline day 3 imputation & $0.206(\mathrm{~N}=686)$ & $0.829(\mathrm{~N}=1532)$ & $0.857(\mathrm{~N}=1175)$ & $0.415(\mathrm{~N}=1227)$ \\
\hline $\begin{array}{l}\text { day 1 imputation } \\
\text { [Ennett, 2003] }\end{array}$ & $0.702(\mathrm{~N}=286)$ & $0.225(\mathrm{~N}=1066)$ & $0.981(\mathrm{~N}=919)$ & $0.341(\mathrm{~N}=689)$ \\
\hline
\end{tabular}

A t-test with a two-tailed sigmoid function was performed for which the $\mathrm{p}$ values are shown in the first row of table 5-4. The $p$ values are statistically acceptable $(p>0.05)$, indicating that the imputed values were similar to the original values. The full $t$-test results are shown in appendix E. Table 5-4 also shows the p values from Ennett's imputation of day 1 data using the CBR tool. Although, there doesn't seem to be a correlation between Ennett's $p$ values and the $p$ values achieved for day 3 for the variables, the $p$ values were generally within a similar range.

The kNN was able to effectively impute the artificially removed values in the day 3 database. It was used in subsequent imputation steps to impute missing values in the day 3 database.

The artificially imputed data using the non-linear network weights was run on the ANN RFW to observe the performance of the $\mathrm{kNN}$ imputed values compared to the 
original database with the true values. The mortality rate was again resampled to $20 \%$ and the results are shown in table 5-5 for ANN mortality prediction.

Table 5-5: ANN performance results for artificial set imputed using non-linear weights $(\mathrm{N}=2,364)$

\begin{tabular}{|l|l|l|l|}
\hline \multicolumn{2}{|l}{} & 2 hidden & 18 hidden \\
\hline Best test epoch & Best test & 1412 & 1412 \\
\cline { 2 - 4 } Log sensitivity & Verification average & 0.072266 & 0.081445 \\
\hline \multirow{3}{*}{ Sensitivity (\%) } & Best test & 38.3 & 40.4 \\
\cline { 2 - 4 } & Verification average & 24.9 & 20.2 \\
\hline \multirow{2}{*}{ Specificity (\%) } & Best test & 92.0 & 91.8 \\
\cline { 2 - 4 } & Verification average & 92.3 & 92.9 \\
\hline Classification Rate (CR) (\%) & 87.4 & 87.4 \\
\hline Area under ROC & 67.4 & 70.5 \\
\hline
\end{tabular}

The results show some degradation when compared to the results in table 5-2 of the original resampled dataset. The degradation is understandable since the imputed values are merely an estimation made by the $\mathrm{kNN}$ of the original value. The highest test sensitivity and average sensitivity have decreased (from $37.0 \%$ to $24.9 \%$ for the verification set average). The area under the ROC has also degraded from the original $71 \%$ to only $67.4 \%$.

\subsection{4 kNN Imputation using Linear weights}

Table 5-2 shows the best performing structures of the ANN RFW where structures with 0 to $19(2 n+1)$ hidden nodes were run. Note that the structure with 0 hidden nodes (a linear or single-layered network) does not appear in the table because it did not perform as well as the non-linear structures. The results from the 2-layered $(0$ hidden node) structure are shown in table 5-6. The sensitivity achieved is considerably less than that achieved using four hidden nodes (22.6\% compared with $37 \%$ ). 
Table 5-6: ANN performance results for imputed database using linear weights $(\mathrm{N}=2,364)$

\begin{tabular}{|l|l|l|}
\hline \multicolumn{2}{|l|}{} & 0 hidden \\
\hline \multicolumn{2}{|l|}{ Best test epoch } & 872 \\
\hline \multirow{2}{*}{ Log sensitivity } & Best test & 0.025136 \\
\cline { 2 - 3 } & Verification average & 0.023857 \\
\hline \multirow{2}{*}{ Sensitivity (\%) } & Best test & 23.4 \\
\cline { 2 - 3 } & Verification average & 22.6 \\
\hline \multirow{2}{*}{ Specificity (\%) } & Best test & 93.6 \\
\cline { 2 - 3 } & Verification average & 93.1 \\
\hline Classification Rate (CR) (\%) & 87.5912 \\
\hline Area under ROC & 0.6926 \\
\hline
\end{tabular}

Although its performance was not as good as the other structures, the weights from the linear network (structure with 0 hidden nodes) were still extracted so that the linear weight imputation could be compared to non-linear weight imputation as per objective 2 of this research. Ennett used weights from linear neural networks in her imputation method since she did not have a method of extracting the weights from a nonlinear network. Since then, MIRG has developed a program to automatically extract nonlinear network weights using the extended Garson-Goh algorithm.

Table 5-7: Variable weights from two-layered network

\begin{tabular}{ll}
\hline Variable & Normalized Weight \\
\hline bthwt & 100 \\
apgar5 & 62 \\
seizure & 43 \\
Itempf & 41 \\
Iserum & 25 \\
po2fio2r & 20 \\
sga & 15 \\
lurine & 3 \\
Ibloodp & 1 \\
\hline
\end{tabular}

Table 5-7 shows the variable weights from the 2-layered network. These weights were extracted easily because they were taken directly from the ANN and did not have to 
be processed using an algorithm such as that required for extracting weights from a 3layered network.

One noticeable difference between the linear weights shown in table 5-8 and nonlinear weights (table 5-3) is that the linear weights have a broad range from weight 1 to weights 62 and then 100, whereas the non-linear weights are clustered between 46 and 100 (with 27 being the lowest).

The other difference can be seen in table 5-8 that shows the variable order. The order for the linear network is quite different from the non-linear. The least significant variables apgar 5, seizure and ltempf in the non-linear network are more significant in the linear network, while some significant variables (po2fio2r and lserum) of the non-linear network are lower in significance in the linear network. Bthwt has remained fairly high in significance in both cases.

Table 5-8: SNAPPE-II variable order of importance (starting with most important)

\begin{tabular}{ll}
\hline $\begin{array}{l}\text { Linear network } \\
\text { hidden nodes) }\end{array}$ & $\begin{array}{l}\text { Non-linear network } \\
\text { (4 hidden nodes) }\end{array}$ \\
\hline bthwt & po2fio2r \\
apgar5 & Iserum \\
seizure & bthwt \\
Itempf & Ibloodp \\
Iserum & sga \\
po2fio2r & lurine \\
sga & apgar5 \\
lurine & seizure \\
lbloodp & Itempf \\
\hline
\end{tabular}

Imputation was performed using the $\mathrm{kNN}$ tool and linear weights as was done for the non-linear weights. Table 5-9 shows the t-test results of the kNN imputation for each missing variable. 
Table 5-9: T test (sig-2) results for imputed datasets using linear and non-linear weights

\begin{tabular}{|l|l|l|l|l|}
\hline & $\begin{array}{l}\text { Ibloodp } \\
(\mathbf{N}=686)\end{array}$ & $\begin{array}{l}\text { po2fio2r } \\
(\mathbf{N = 1 5 3 2 )}\end{array}$ & $\begin{array}{l}\text { lurine } \\
(\mathbf{N = 1 1 7 5 )}\end{array}$ & $\begin{array}{l}\text { Iserum } \\
(\mathbf{N}=1227)\end{array}$ \\
\hline Linear (0 hidden) & 0.230 & 0.710 & 0.811 & 0.766 \\
\hline Non-linear (4 hidden) & 0.206 & 0.829 & 0.857 & 0.415 \\
\hline
\end{tabular}

The $p$ values shown for the imputation using linear weights are statistically acceptable again $(\mathrm{p}>0.05)$. The $\mathrm{p}$ values for both imputation methods are quite close. The linear weight imputation gave significantly better imputation results for lserum and slightly lower results for po2fio2r, while lbloodp and lurine results were almost the same as the non-linear imputation. The complete t-test results are shown in appendix E.

A comparison of the variable means was also done as shown in table 5-10. The means for the non-linear imputation seem to be closer to the original for lbloodp and po2fio2r and the means of the linear and non-linear imputation methods are quite similar for lurine and lserum. The standard deviation for both imputation methods is less than the standard deviation of the original data. This is understandable because the imputed values are computed from the mean of the closest matching values, therefore the imputed values do not end up deviating notably from the mean.

Table 5-10: Mean and standard deviation comparison of original dataset with imputed datasets

\begin{tabular}{|l|l|l|l|l|}
\hline & Ibloodp & po2fio2r & lurine & Iserum \\
\hline True values & $37.1778 \pm 8.86$ & $2.0538 \pm .94$ & $3.7332 \pm 1.65$ & $7.3131 \pm .08$ \\
\hline Linear imputation & $37.5356 \pm 6.08$ & $2.0633 \pm .31$ & $3.7451 \pm .52$ & $7.3125 \pm .04$ \\
\hline $\begin{array}{l}\text { Non-linear (4 hidden) } \\
\text { imputation }\end{array}$ & $37.1778 \pm 6.09$ & $2.0593 \pm .32$ & $3.7421 \pm .51$ & $7.3114 \pm .04$ \\
\hline
\end{tabular}

The artificially imputed database was run on the ANN RFW to observe the performance of the imputed values using linear weights compared to the imputed database using non-linear weights and the original database with the true values. 
Table 5-11: ANN performance results for imputed dataset using linear weights $(\mathrm{N}=13,871)$

\begin{tabular}{|l|l|l|l|l|}
\hline \multicolumn{2}{|l}{} & 14 hidden & 15 hidden & 19 hidden \\
\hline Best test epoch & 613 & 1034 & 107 \\
\hline \multirow{2}{*}{ Log sensitivity } & Best test & 0.082575 & 0.051477 & 0.047939 \\
\cline { 2 - 5 } & Verification average & $\mathbf{0 . 0 3 6 3 8 5}$ & 0.026555 & 0.031579 \\
\hline \multirow{3}{*}{ Sensitivity (\%) } & Best test & 40.4 & 34.0 & 31.9 \\
\cline { 2 - 5 } & Verification average & 27.9 & 23.8 & 25.9 \\
\hline \multirow{2}{*}{ Specificity (\%) } & Best test & 92.8 & 91.4 & 91.6 \\
\cline { 2 - 5 } & Verification average & 91.4 & 91.7 & 91.5 \\
\hline Classification Rate (CR) (\%) & 88.3 & 86.5 & 86.5 \\
\hline Area under ROC & 68.1 & 64.5 & 62.2 \\
\hline
\end{tabular}

The results of the ANN RFW on the imputed data using linear weights in table 5-11 above, were close to the results of the imputed data using non-linear weights shown in table 5-5. The results of the imputation using linear weights gave a higher sensitivity than imputation using non-linear weights (27.9\% compared to $24.9 \%)$. The specificity, CR and area under ROC are almost the same for both. Because the linear weight imputation was able to achieve a higher sensitivity than the non-linear imputation method, the linear weights were used for imputation in the vertical and horizontal imputation steps.

After examining these results, the question arises: how do the linear weights give a better ANN result after imputation when the linear ANN structure (in table 5-6) was not able to perform as well as the non-linear network (shown in 5-2)? One explanation is that the value and order of the weights used in $\mathrm{kNN}$ is not important in the imputation process. Ennett's results demonstrate this. Ennett investigated the imputation of missing values using ANN weights (as done in this research) compared with setting all the weights to 100 ("uniform weights") and setting the perinatal extension variables (sga, bthwt and apgar 5) to 100 while the rest of the variables were set to the ANN weights ("clinician weights"). Ennett's results are shown in table 5-12. The ANN weight imputation was comparable to the uniform imputation and clinician weight imputation methods. She also concluded that the hybrid approaches (ANN weights, clinician 
weights and uniform weights) were all better than mean and random imputations approaches.

Table 5-12: Ennett's ANN performance results for day 1 data using the best test set performance $(\mathrm{N}=5,102)$

\begin{tabular}{lllllll}
\hline $\begin{array}{l}\text { Performance } \\
\text { measure }\end{array}$ & $\begin{array}{l}\text { True } \\
\text { values }\end{array}$ & $\begin{array}{l}\text { ANN } \\
\text { weights }\end{array}$ & $\begin{array}{l}\text { Clinician } \\
\text { weights }\end{array}$ & $\begin{array}{l}\text { Uniform } \\
\text { weights }\end{array}$ & Mean & Random \\
\hline $\begin{array}{l}\text { Sensitivity (\%) } \\
\text { Specificity (\%) }\end{array}$ & 25.7 & $\mathbf{2 4 . 0}$ & 24.0 & 23.5 & 22.3 & 22.9 \\
$\begin{array}{l}\text { Classification } \\
\text { rate (\%) }\end{array}$ & 90.2 & $\mathbf{9 6 . 8}$ & 96.8 & 97.0 & 97.4 & 96.9 \\
$\begin{array}{l}\text { Area under ROC } \\
\text { curve }\end{array}$ & $\mathbf{0 . 8 2 9 0}$ & $\mathbf{0 . 7 7 8 6}$ & $\mathbf{0 . 7 6 9 7}$ & 0.7814 & 0.7815 & 0.7701 \\
\hline
\end{tabular}

Therefore, the results obtained in this research are in accordance with Ennett's results. So, the linear weights were used for subsequent imputations. Another reason for choosing imputation using linear weights is that the linear weights are easily obtained because they are the weights given directly by the ANN.

\subsection{Vertical Expansion}

The weights from the two-layered network in step 1 (shown in table 24) are used to vertically expand the database so that any missing variables of the 9 SNAPPE-II variables are imputed for all the cases in the day 3 database $(13,871)$. The following table 5-13 shows the increase in the mean values as cases with more missing values are imputed and added to the match set. The mean values of lbloodp, po2fio2r, lserum and $b t h w t$ all increase considerably as cases with more missing values are imputed. This indicates that the infants with more missing values have larger values for these variables and therefore, the infants with the least missing values were in fact the sickest of the population (i.e. had lower mean values for all variables). 
Table 5-13: Mean and standard deviation changes in the complete dataset as cases missing an increasing number of missing values are added

\begin{tabular}{|l|l|l|l|l|l|}
\hline & Ibloodp & po2fio2r & lurine & lserum & bthwt \\
\hline $\begin{array}{l}\text { Complete } \\
\text { cases }\end{array}$ & $37.4924 \pm 8.95$ & $2.0544 \pm .95$ & $3.7345 \pm 1.67$ & $7.3143 \pm .08$ & $1816.7961 \pm 1083.00$ \\
\hline $1 \mathrm{mv}^{*}$ & $39.2469 \pm 9.16$ & $2.0715 \pm .80$ & $3.7088 \pm 1.55$ & $7.3247 \pm .08$ & $1886.7252 \pm 1039.98$ \\
\hline $2 \mathrm{mv}^{*}$ & $41.3875 \pm 9.47$ & $2.0761 \pm .67$ & $3.6557 \pm 1.58$ & $7.3309 \pm .07$ & $1971.0201 \pm 1020.14$ \\
\hline $3 \mathrm{mv}^{*}$ & $43.7073 \pm 9.76$ & $2.0757 \pm .58$ & $3.6392 \pm 1.36$ & $7.3358 \pm .06$ & $2091.4616 \pm 995.26$ \\
\hline $4 \mathrm{mv}^{*}$ & $44.4552 \pm 8.58$ & $2.0760 \pm .52$ & $3.6352 \pm 1.19$ & $7.3397 \pm .05$ & $2222.7129 \pm 973.66$ \\
\hline
\end{tabular}

*Where $\mathrm{mv}$ is an abbreviation for the number of missing values in each case of the query set

The results from the ANN RFW performance on the full 13,871 day 3 cases of the 9 SNAPPE-II variables are shown in table 5-14. A significant increase in ANN performance is apparent when the performance of the 13,871 cases in table 5-14 is compared to the performance of 2,364 cases in table 5-2. Both sets have all 9 SNAPPEII variable values. However, an average sensitivity of $52.6 \%$ was achieved with the full set of cases compared to the $37.0 \%$ with the 2,364 cases. The CR also increased slightly to 90.3 (from 87.6) and the area under the ROC increased significantly to 84.5 (from 71.8). As was stated in section 2.1.2, the aim is to get a sensitivity of at least $50 \%$ and specificity of at least $90 \%$. The results of the three-layered network using the 9 SNAPPE-II variables gives a specificity and sensitivity that was just above the target. These results demonstrate that when more cases are used to train the ANN, a higher sensitivity and better overall performance is achieved. Thus replacing missing values with our hybrid system is a good approach to improve ANN performance.

Ennett's results are shown in table 5-15 in which her hybrid model's performance on the ANN is compared with the SNAPPE-II model's performance. The results from table 5-14 are difficult to compare to Ennett's in table 5-15 because the specificity of the ANN used by Ennett was set to $98.9 \%$, whereas the specificity was set to anything over $90 \%$ for the ANN RFW used in this research. 
Table 5-14: ANN performance results for vertically imputed database of SNAPPE-II variables $(\mathrm{N}=13,871)$

\begin{tabular}{|l|l|l|l|l|}
\hline \multicolumn{2}{|l}{2 hidden } & 9 hidden & 15 hidden \\
\hline Best test epoch & 338 & 1617 & 182 \\
\hline \multirow{2}{*}{ Log sensitivity } & Best test & 0.2782 & 0.20255 & 0.19315 \\
\cline { 2 - 5 } & Verification average & $\mathbf{0 . 1 5 2 8 3}$ & 0.13101 & 0.119773 \\
\hline \multirow{2}{*}{ Sensitivity (\%) } & Best test & 67.4 & 59.3 & 58.1 \\
\cline { 2 - 5 } & Verification average & 52.6 & 49.2 & 47.6 \\
\hline \multirow{2}{*}{ Specificity (\%) } & Best test & 90.9 & 91.8 & 92.0 \\
\cline { 2 - 5 } & Verification average & 90.7 & 91.1 & 91.0 \\
\hline Classification Rate (CR) (\%) & 90.3 & 90.9 & 91.0 \\
\hline Area under ROC & 84.5 & 85.3 & 83.0 \\
\hline
\end{tabular}

To be able to compare the SNAPPE-II model to Ennett's, the complete cases of the day 3 database $(\mathrm{N}=2,364)$ are used as the match set to impute the 13 variables identified by Ennett, similar to the imputation of the 9 SNAPPE-II variables above. The 13 variables are imputed for all 13,871 cases of the day 3 database. The ANN results for the full set of cases for 13 variables are shown in table 5-16.

Table 5-15: Ennett's results for 13 input variables [Ennett, 2003]

\begin{tabular}{|c|c|c|c|c|c|}
\hline Performance measure & $\begin{array}{l}\text { Hybrid } \\
\text { model }\end{array}$ & $\begin{array}{l}\text { SNAP } \\
\text { cutoff } \\
=30\end{array}$ & $\begin{array}{l}\text { SNAPPE } \\
\text { cutoff } \\
=56\end{array}$ & $\begin{array}{l}\text { SNAP-II } \\
\text { cutoff } \\
=42\end{array}$ & $\begin{array}{l}\text { SNAPPE-II } \\
\text { cutoff } \\
=65\end{array}$ \\
\hline \multicolumn{6}{|l|}{ SPECIFICITY $=98.9 \%$} \\
\hline Sensitivity (\%) & 38.3 & 23.0 & 23.4 & 30.4 & 31.6 \\
\hline Specificity (\%) & 98.9 & 98.7 & 98.8 & 98.9 & 98.9 \\
\hline Classification rate $(\%)$ & 96.8 & 95.9 & 96.0 & 96.3 & 96.4 \\
\hline SENSITIVITY $=80.0 \%$ & & $\begin{array}{l}\text { Cutoff } \\
=15\end{array}$ & $\begin{array}{l}\text { Cutoff } \\
=24\end{array}$ & $\begin{array}{l}\text { Cutoff } \\
=13\end{array}$ & $\begin{array}{l}\text { Cutoff } \\
=28\end{array}$ \\
\hline Sensitivity (\%) & 80.1 & 80.6 & 79.1 & 79.9 & 79.5 \\
\hline Specificity (\%) & 84.4 & 70.4 & 84.1 & 77.8 & 85.4 \\
\hline Classification rate (\%) & 84.2 & 70.8 & 84.0 & 77.9 & 85.2 \\
\hline Area under ROC curve & 0.8699 & & & & $\begin{array}{l}0.89 \pm 0.01^{a} \\
0.91 \pm 0.01^{\mathrm{b}}\end{array}$ \\
\hline
\end{tabular}

${ }^{a}$ From Richardson et al. [2001], test set performance, $N=5588$ cases.

${ }^{\mathrm{b}}$ From Richardson et al. [2001], training set performance, $N=10819$ cases. 
Table 5-16: ANN performance results for 13 variable Ennett model on day 3 data $(\mathrm{N}=13,871)$

\begin{tabular}{|l|l|l|l|l|}
\hline \multicolumn{2}{|l}{0 hidden } & 9 hidden & 12 hidden \\
\hline Best test epoch & 786 & 573 & 147 \\
\hline \multirow{2}{*}{ Log sensitivity } & Best test & 0.29583 & 0.28151 & 0.28448 \\
\cline { 2 - 5 } & Verification average & $\mathbf{0 . 2 8 3 7 1 4}$ & 0.223795 & 0.242818 \\
\hline \multirow{2}{*}{ Sensitivity (\%) } & Best test & 69.2 & 67.9 & 67.9 \\
\cline { 2 - 5 } & Verification average & 67.8 & 61.9 & 63.6 \\
\hline \multirow{2}{*}{ Specificity (\%) } & Best test & 90.4 & 90.5 & 91.0 \\
\cline { 2 - 5 } & Verification average & $\mathbf{9 0 . 5}$ & 90.8 & 90.7 \\
\hline Classification Rate (CR) (\%) & 89.9 & 89.9 & 90.5 \\
\hline Area under ROC & 85.2 & 83.7 & 84.4 \\
\hline
\end{tabular}

The results of table 5-16 show that the 13 variable dataset performed better than the 9 variable set of SNAPPE-II variables in table 5-14. The average specificity has increased to $67.8 \%$ from $52.6 \%$ and the area under the ROC has increased from 84.5 to 85.2. The specificity and CR have remained essentially the same. The ROC figures for the best performing structure for the SNAPPE-II variable set and for the 13 variable Ennett model are shown in appendix $B$ as well as the default parameters used for the ANN RFW. Although, the structures containing 9 and 12 hidden nodes achieved a very high sensitivity for the best test epoch, they did not achieve an average sensitivity that was as high as the two-layered structure (with 0 hidden nodes). This can be expected, since Ennett's model was developed using weights from 2-layered networks only. The SNAPPE-II model was developed using a logistic regression method that is likened to a two-layered neural network [Richardson et al., 1993]. However, it did not obtain its highest sensitivity on a two-layered structure but on the 3-layered structure containing two hidden nodes as shown in table 5-14.

The results of the SNAPPE-II variable model and Ennett's 13 variable models are compared with the new models developed specifically for day 3 in the following section. 


\subsection{Horizontal Expansion}

The weights for each set of variables in section 3.3.1 were derived from the twolayered structure that gave the best performance for predicting mortality. A 2-layered structure is used as opposed to a 3-layered structure (containing a hidden layer) because it was concluded in section 4.1 that data imputed by the kNN using linear weights gave a better performance than non-linear weights. The variable sets that were used as input variables to the network in order to derive the weights, were as follows:

- Set A (23 input variables, 1700 cases, mortality rate =9.6\%);

- Set B (25 input variables, 1077 cases, mortality rate $=10.2 \%$ ); and

- Set C (28 input variables, 552 cases, mortality rate $=13.2 \%$ ).

The weights of these variable sets are shown in table 5-17. There are some variables that seem to remain quite significant such as lpo2po2, hpotass, bthwt, and hheartr. Others seem to fluctuate such as lbloodp which has a high weight in set A, becomes quite low in set $\mathrm{C}$. Weights of 0 were changed to 1 when entered into the $\mathrm{kNN}$ because the $\mathrm{kNN}$ does not accept weights below 1. The SNAPPE-II weights which are bolded in the table, appear at different weights and are not clustered at the top where one might think they would appear.

The weights obtained were quite different from those obtained by Ennett for the day 1 database.

These weights were used to gradually expand the database 'horizontally' i.e. so that there were more variables for each case. The expansion was performed gradually as shown in figure 4-1, starting with the cases with the least missing values imputed first to gradually influence the mean of the dataset until all 28 variables had been imputed for the 2,364 cases of the day 3 database. 
Table 5-17: Variable weights used in horizontal imputation

\begin{tabular}{|c|c|c|c|c|c|}
\hline \multicolumn{2}{|c|}{ Set $A$ weights } & \multicolumn{2}{|c|}{ Set B weights } & \multicolumn{2}{|c|}{ Set $C$ weights } \\
\hline Iheartr & 100 & hpco2 & 100 & Iserum & 100 \\
\hline po2fio $2 r$ & 85 & bthwt & 100 & hheartr & 82 \\
\hline Ibloodp & 62 & oi & 86 & Iplt & 78 \\
\hline Ipo2po2 & 61 & Iserum & 83 & hpco2 & 65 \\
\hline apgar5 & 51 & hheartr & 74 & 1po2po2 & 56 \\
\hline hpotass & 50 & hpotass & 62 & hsodium & 49 \\
\hline hrespr & 43 & Ipo2po2 & 59 & lurine & 46 \\
\hline guaiac & 40 & hidbili & 54 & |potass & 38 \\
\hline bthwt & 25 & apgar5 & 40 & hpotass & 31 \\
\hline seizure & 24 & Ibloodp & 36 & Isodium & 30 \\
\hline hgluc & 19 & guaiac & 33 & bthwt & 30 \\
\hline hheartr & 18 & Iheartr & 32 & hbloodp & 28 \\
\hline hbloodp & 16 & hsodium & 32 & apnea & 27 \\
\hline Isodium & 14 & Igluc & 26 & po2fio $2 r$ & 24 \\
\hline Igluc & 14 & po2fio2r & 20 & apgar5 & 22 \\
\hline Itempf & 13 & seizure & 16 & hhema & 21 \\
\hline hsodium & 11 & hbloodp & 15 & Iwbc & 21 \\
\hline sga & 10 & hrespr & 15 & seizure & 19 \\
\hline Ipotass & 9 & Itempf & 15 & Igluc & 16 \\
\hline apnea & 7 & Ipotass & 15 & oi & 1 \\
\hline Iserum & 4 & lurine & 14 & Ibloodp & 0 \\
\hline hpco 2 & 3 & Isodium & 14 & Iheartr & 0 \\
\hline \multirow[t]{6}{*}{ lurine } & 1 & sga & 10 & hrespr & 0 \\
\hline & & apnea & 9 & Itempf & 0 \\
\hline & & hgluc & 5 & hidbili & 0 \\
\hline & & & & hgluc & 0 \\
\hline & & & & guaiac & 0 \\
\hline & & & & sga & 0 \\
\hline
\end{tabular}

\subsection{Finding the Model}

\subsubsection{Using linear structure to find Minimum Data Set}

The horizontally expanded dataset consisting of 2,364 cases with 28 variables present for each case was run on the ANN RFW tool to predict mortality. The performance of the ANN was analyzed and the variable weights were examined. The variables with the smallest weights were the least important to the network and so they were gradually removed. This process was repeated until a point was reached when the 
network could not converge. The variable set that gave the highest ANN performance and that ensured the network converged, was chosen as the 'minimum dataset'.

Table 5-18 shows the ANN performance of the network as variables were gradually removed.

Table 5-18: ANN performance results on two-layered network as variables are gradually removed $(\mathrm{N}=2,364)$

\begin{tabular}{|c|c|c|c|c|c|c|c|c|}
\hline \multirow{2}{*}{\multicolumn{2}{|c|}{\begin{tabular}{|l|l|} 
Best test epoch \\
\end{tabular}}} & 28 & $\begin{array}{l}22 \\
\text { variables }\end{array}$ & variables & $\begin{array}{l}15 \\
\text { variables }\end{array}$ & $\begin{array}{l}14 \\
\text { variables }\end{array}$ & $\begin{array}{l}13 \\
\text { variables }\end{array}$ & $\begin{array}{l}12 \\
\text { variables }\end{array}$ \\
\hline & & 228 & 387 & 243 & 694 & 572 & 717 & 329 \\
\hline \multirow{2}{*}{$\begin{array}{l}\log \\
\text { sensitivity }\end{array}$} & Best test & 0.05394 & 0.083237 & 0.048058 & 0.094944 & 0.087332 & 0.10417 & 0.056005 \\
\hline & $\begin{array}{l}\text { Verification } \\
\text { average }\end{array}$ & 0.114155 & 0.10417 & 0.086651 & 0.09822 & 0.077636 & 0.075733 & 0.062738 \\
\hline \multirow{2}{*}{$\begin{array}{l}\text { Sensitivity } \\
(\%)\end{array}$} & Best test & 33.9 & 45.2 & 32.3 & 43.5 & 41.9 & 45.2 & 33.9 \\
\hline & $\begin{array}{l}\text { Verification } \\
\text { Average }\end{array}$ & 46.2 & 40.5 & 40.8 & 43.6 & 39.1 & 38.5 & 34.7 \\
\hline \multirow{2}{*}{$\begin{array}{l}\text { Specificity } \\
(\%)\end{array}$} & Best test & 90.6 & 91.2 & 90.0 & 90.6 & 90.8 & 91.2 & 93.5 \\
\hline & $\begin{array}{l}\text { Verification } \\
\text { Average }\end{array}$ & 91.5 & 91.1 & 90.4 & 91.3 & 91.5 & 91.3 & 91.8 \\
\hline \multicolumn{2}{|c|}{ Classification Rate (CR) $(\%)$} & 84.3 & 86.1 & 83.5 & 85.4 & 85.4 & 86.1 & 86.8 \\
\hline \multirow{2}{*}{$\begin{array}{l}\text { Area under } \\
\text { ROC }\end{array}$} & Best test & 71.8 & 75.5 & 75.3 & 71.4 & 75.2 & 77.2 & 73.4 \\
\hline & $\begin{array}{l}\text { Verification } \\
\text { Average }\end{array}$ & 77.8 & 75.9 & 80.0 & 72.2 & 72.5 & 79.1 & 74.8 \\
\hline
\end{tabular}

The table above shows that the ANN is able to attain a fairly high average sensitivity and area under the ROC with the 28 variables ( $46.2 \%$ and 77.8 respectively). As variables are reduced to 22 and then 16 variables the average sensitivity decreases slightly. The ANN performance of 15 variables achieves a high average sensitivity (43.6). This increase in performance over the 22 and 16 variable set can be explained because less noise may exist in the system as the least significant variables are removed. As more noise is removed the performance is expected to improve but only up to a certain point after which any further removal of variables will cause degradation. The removal of the least significant variable from the set of 13 variables resulted in a network with a significantly lower average sensitivity (34.7\%). So, the 13 variable dataset was kept as the minimum because it was able to attain an acceptable average sensitivity (38.5\%) and high average area under the ROC (79.1). 
Table 5-19: Variable weights on linear network as least important variables are gradually removed

\begin{tabular}{|c|c|c|c|c|c|c|c|c|c|c|c|}
\hline \multicolumn{2}{|c|}{28 variables } & \multicolumn{2}{|c|}{22 variables } & \multicolumn{2}{|c|}{16 variables } & \multicolumn{2}{|c|}{15 variables } & \multicolumn{2}{|c|}{14 variables } & \multicolumn{2}{|c|}{13 variables } \\
\hline Ipo2po2 & 100 & Iheartr & 100 & guaiac & 100 & guaiac & 100 & Iserum & 100 & Iheartr & 100 \\
\hline lurine & 92 & guaiac & 97 & hrespr & 19 & po2fio $2 r$ & 39 & Ipo2po2 & 60 & Isodium & 82 \\
\hline apgar5 & 84 & hrespr & 53 & hhema & 14 & Ibloodp & 20 & apgar5 & 52 & hrespr & 73 \\
\hline hrespr & 82 & hheartr & 39 & Iheartr & 13 & Iheartr & 17 & Iheartr & 38 & po2fio $2 r$ & 72 \\
\hline guaiac & 81 & Iserum & 35 & Ipo2po2 & 12 & hrespr & 13 & hrespr & 38 & Ibloodp & 65 \\
\hline Iheartr & 76 & po2fio $2 r$ & 33 & po2fio $2 r$ & 12 & Ipotass & 12 & seizure & 38 & hhema & 57 \\
\hline Isodium & 74 & seizure & 33 & Isodium & 12 & apgar5 & 9 & guaiac & 36 & Ipo2po2 & 51 \\
\hline hheartr & 71 & hgluc & 31 & apgar5 & 11 & hgluc & 8 & hhema & 30 & apgar5 & 47 \\
\hline hgluc & 57 & apgar5 & 29 & seizure & 10 & lurine & 7 & Ipotass & 29 & guaiac & 46 \\
\hline hhema & 54 & Itempf & 23 & lurine & 7 & Iserum & 6 & po2fio $2 r$ & 26 & Ipotass & 45 \\
\hline Ibloodp & 51 & Ipotass & 21 & Iserum & 7 & seizure & 6 & Ibloodp & 16 & seizure & 43 \\
\hline seizure & 45 & hhema & 20 & hgluc & 6 & Ipo2po2 & 5 & Isodium & 14 & lurine & 34 \\
\hline po2fio2r & 44 & Ipo2po2 & 18 & Ibloodp & 4 & hhema & 4 & lurine & 10 & Iserum & 22 \\
\hline oi & 42 & lurine & 16 & hheartr & 4 & hheartr & 3 & hgluc & 6 & & \\
\hline hidbili & 40 & Ibloodp & 15 & Itempf & 2 & Isodium & 3 & & & & \\
\hline Ipotass & 39 & Isodium & 13 & Ipotass & 2 & & & & & & \\
\hline bthwt & 39 & $o i$ & 10 & & & & & & & & \\
\hline Iplt & 35 & Iplt & 10 & & & & & & & & \\
\hline Itempf & 34 & bthwt & 9 & & & & & & & & \\
\hline hpotass & 34 & hpotass & 7 & & & & & & & & \\
\hline Iwbc & 33 & Iwbc & 4 & & & & & & & & \\
\hline Iserum & 30 & hidbili & 4 & & & & & & & & \\
\hline Igluc & 27 & & & & & & & & & & \\
\hline hsodium & 23 & & & & & & & & & & \\
\hline sga & 22 & & & & & & & & & & \\
\hline hbloodp & 17 & & & & & & & & & & \\
\hline hpco2 & 8 & & & & & & & & & & \\
\hline apnea & 2 & & & & & & & & & & \\
\hline
\end{tabular}

Table 5-19 shows the variable weights from the most significant in predicting mortality on day 3 data to the least significant. Table 5-19 also shows which variables were removed; these are shown in italics in the table. When 16 variables remained, variables were removed one by one. For example, in the 16 variable set lpotass was removed but a significant degradation was obtained (average sensitivity of only $37 \%$ ) so it was kept in the model and ltempf was removed instead. Similarly in the 15 variable set the removal of lsodium resulted in greater degradation than the removal of hhema and so hhema was removed and lsodium was kept. It would be ideal to remove each of the 28 
variables one by one to ensure variables that are important to the network are not removed, but that would take a very long time.

Certain variables such as hrespr, lpo2po2, hheartr, and guaiac remained quite high in all iterations. Other variables such as lserum and lurine were high in some iterations but lower in others. It was strange that seizure was not a very significant variable in all of the iterations and is not a common occurrence, but it still managed to avoid elimination and remain in the minimum dataset. The full variable reduction results are shown in appendix $\mathrm{C}$.

The final minimum dataset that resulted from variable reduction of the linear network is shown in table 5-20 next to the day 1 mortality model found by Ennett. It was surprising to note that bthwt was eliminated from the day 3 model even though it is deemed an important variable in predicting mortality [Fowlie et al., 1998] at least in the first 12 hours after birth. However, it was noted that the bthwt variable records the infant's weight at birth and not the current weight as it changes day to day as initially thought. This is also the case with the variables apgar5 and sga - the other perinatal extension variables. Because these variables are entered into the database upon admission to the NICU and do not change after admission, it is understandable that their significance in predicting mortality will diminish with time. It should also be noted that the SNAP and SNAP-II models, which are fairly widely used currently in mortality prediction, do not contain these three perinatal extensions variables. Also observed was that in Ennett's model bthwt is quite low in the variable importance (with a weight of only 21), although apgar 5 is the fourth most significant variable in her model.

It is also observed that sga and ltempf were removed from the day 3 model while they all exist in the day 1 model. Of the variables that are not in the SNAPPE-II set, the only similar one between the day 1 and day 3 models is hrespr. None of lplt, hsodium, hpco 2 or lgluc from the day 1 model were kept in the day 3 model. Lpo2po2 that appears in the day 3 model, contains respiratory information that could be used in place of the variable $h p c o 2$ from the day 1 model. 
Table 5-20: A comparison of the 10 variable day 3 linear model, the 13 variable day 1 model found by Ennett and the SNAPPE-II model

\begin{tabular}{llllll}
\hline Linear Model & \multicolumn{2}{l}{ Ennett Model } & SNAPPE-II \\
\hline Iheartr & 100 & po2fio2r & 100 & bthwt & 100 \\
Isodium & 82 & lurine & 72 & apgar5 & 62 \\
hrespr & 73 & Iserum & 36 & seizure & 43 \\
po2fio2r & 72 & apgar5 & 33 & Itempf & 41 \\
Ibloodp & 65 & Iplt & 29 & Iserum & 25 \\
hhema & 57 & sga & 28 & po2fio2r & 20 \\
Ipo2po2 & 51 & hsodium & 28 & sga & 15 \\
apgar5 & 47 & hrespr & 24 & lurine & 3 \\
guaiac & 46 & hpco2 & 22 & Ibloodp & 1 \\
Ipotass & 45 & bthwt & 21 & & \\
seizure & 43 & Igluc & 20 & & \\
lurine & 34 & Itempf & 18 & & \\
Iserum & 22 & hbloodp & 18 & & \\
\hline
\end{tabular}

\subsubsection{Using a Non-linear Structure to find Minimum Data Set}

The same method that was used to find the minimum dataset above was used again but on 3-layered networks. This step was different from Ennett's research, in which she only found the minimum data set of a 2-layered network. It is an important step because medical data is non-linear in nature [Frize et al., 2001], so a non-linear network should be able to determine the non-linear relationships between the variables and therefore, should give a better performance and a more meaningful variable set than a linear network is able to.

Table 5-21 shows the ANN RFW performance on 3-layered structures. The structures 0 to $2 n+1$ were attempted, where $n$ was the number of input variables that decreased with each iteration as variables were removed. The second row of the table shows which structure gave the best performance for that particular variable set. The average sensitivity achieved increased as the variables were removed from the 28 variable model to a 16 variable model. After that point the average sensitivity decreased steadily until the 13 variable model had a significantly lower average sensitivity $(37.8 \%)$ than the original dataset and less than the 13 variable model reduced in the linear network reduction in table 5-18 (38.5\%). However, the average area under the ROC improved with the 13 variable model compared to most of the other datasets and the highest 
sensitivity achieved was the same as that obtained with the 13 variable linear model in table 5-18. Although table 5-21 shows degradation begins after the 16 variable set is reduced, the performance of the 13 variable set is still acceptable and will therefore be compared with the performance of the 13 variable model obtained from the linear reduction (shown in table 5-20).

Table 5-21: ANN performance results using non-linear network as variables are gradually removed $(\mathrm{N}=2,364)$

\begin{tabular}{|c|c|c|c|c|c|c|c|c|}
\hline 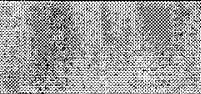 & 20 & rartables & Varables & $\begin{array}{l}16 \\
\text { variables }\end{array}$ & $\begin{array}{l}15 \\
\text { variables }\end{array}$ & $\begin{array}{l}14 \\
\text { Varlables }\end{array}$ & $\begin{array}{l}13 \\
\text { variables }\end{array}$ & $\begin{array}{l}12 \\
\text { variables }\end{array}$ \\
\hline \multicolumn{2}{|l|}{ Structure } & 19 & 11 & 17 & 19 & 16 & 14 & 16 \\
\hline \multicolumn{2}{|c|}{ Best test epoch } & 168 & 254 & 796 & 142 & 280 & 1787 & 479 \\
\hline \multirow{2}{*}{$\begin{array}{l}\log \\
\text { sensitivity }\end{array}$} & Best test & 0.08658 & 0.080332 & 0.11136 & 0.1051 & 0.12241 & 0.10417 & 0.13026 \\
\hline & $\begin{array}{l}\text { Verification } \\
\text { average }\end{array}$ & 0.107097 & 0.102742 & 0.109105 & 0.094892 & 0.082428 & 0.072806 & 0.066516 \\
\hline \multirow{2}{*}{$\begin{array}{l}\text { Sensitivity } \\
(\%)\end{array}$} & Best test & 41.9 & 40.3 & 46.8 & 45.2 & 48.4 & 45.2 & 50.0 \\
\hline & $\begin{array}{l}\text { Verification } \\
\text { Average }\end{array}$ & 45.1 & 44.2 & 45.8 & 42.7 & 40.6 & 37.8 & 36.3 \\
\hline \multirow{2}{*}{$\begin{array}{l}\text { Specificity } \\
(\%)\end{array}$} & Best test & 90.2 & 91.2 & 90.2 & 91.9 & 91.2 & 91.2 & 90.2 \\
\hline & $\begin{array}{l}\text { Verification } \\
\text { Average }\end{array}$ & 90.8 & 90.9 & 91.0 & 91.3 & 90.7 & 93.2 & 91.0 \\
\hline \multicolumn{2}{|c|}{ Classification Rate (CR) $(\%)$} & 84.8 & 85.5 & 85.4 & 86.6 & 86.4 & 86.1 & 85.7 \\
\hline \multirow{2}{*}{$\begin{array}{l}\text { Area under } \\
\text { ROC }\end{array}$} & Best test & 78.3 & 76.9 & 74.2 & 75.8 & 78.0 & 80.2 & 77.1 \\
\hline & $\begin{array}{l}\text { Verification } \\
\text { Average }\end{array}$ & 78.0 & 75.5 & 75.2 & 75.0 & 74.2 & 76.4 & 70.0 \\
\hline
\end{tabular}

The variables were removed in the same manner as they were removed for the linear model in table 5-19. Table 5-22 shows the variable importance in predicting mortality on day 3 data and using a 3-layered network. The variable po2fio $2 r$ which appears in the SNAPPE-II model, Ennett's model and the 10 variable model found on a two-layered network in section 5.4.1 was removed in the second iteration of variable reduction. It is possible however that $l p o 2 p o 2$ and $h p c o 2$ contain enough respiratory information for the network to disregard the information given by po2fio2r. 
Table 5-22: Variable weights for non-linear network as least important variables are gradually removed

\begin{tabular}{|c|c|c|c|c|c|c|c|c|c|c|c|}
\hline \multicolumn{2}{|c|}{28 variables } & \multicolumn{2}{|c|}{22 variables } & \multicolumn{2}{|c|}{16 variables } & \multicolumn{2}{|c|}{15 variables } & \multicolumn{2}{|c|}{14 variables } & \multicolumn{2}{|c|}{13 variables } \\
\hline hrespr & 100 & Ipotass & 100 & hpco2 & 100 & hrespr & 100 & apnea & 100 & hrespr & 100 \\
\hline Igluc & 86 & Iserum & 97 & Ipotass & 94 & hheartr & 98 & hpco2 & 95 & Iplt & 89 \\
\hline hpco2 & 79 & hrespr & 96 & bthwt & 83 & apgar5 & 98 & Iplt & 93 & Ipotass & 81 \\
\hline Isodium & 76 & hheartr & 79 & lbloodp & 78 & hpco2 & 97 & hrespr & 92 & hheartr & 69 \\
\hline Iplt & 75 & guaiac & 79 & Iserum & 78 & Ipotass & 91 & apgar5 & 92 & guaiac & 69 \\
\hline lurine & 74 & hpco2 & 77 & hrespr & 76 & bthwt & 88 & hheartr & 88 & bthwt & 69 \\
\hline hgluc & 74 & hgluc & 77 & Ipo2po2 & 72 & guaiac & 87 & Iserum & 84 & Ipo2po2 & 67 \\
\hline apnea & 74 & oi & 74 & lurine & 71 & apnea & 82 & guaiac & 79 & Iserum & 65 \\
\hline Iwbc & 71 & bthwt & 74 & apgar5 & 65 & Ipo2po2 & 75 & Ibloodp & 76 & hpco2 & 61 \\
\hline hheartr & 69 & apnea & 73 & Iplt & 62 & Ibloodp & 74 & lurine & 74 & lbloodp & 50 \\
\hline guaiac & 62 & |po2po2 & 69 & apnea & 61 & hgluc & 67 & bthwt & 74 & lurine & 50 \\
\hline apgar5 & 62 & Ibloodp & 65 & hgluc & 59 & Iserum & 66 & |po2po2 & 72 & apgar5 & 48 \\
\hline hbloodp & 59 & Iplt & 63 & hheartr & 57 & lurine & 64 & hgluc & 70 & apnea & 20 \\
\hline oi & 59 & apgar5 & 59 & oi & 56 & oi & 57 & |potass & 57 & & \\
\hline bthwt & 59 & hhema & 58 & hhema & 52 & Iplt & 54 & & & & \\
\hline Ibloodp & 56 & lurine & 53 & guaiac & 51 & & & & & & \\
\hline hhema & 55 & Igluc & 51 & & & & & & & & \\
\hline hidbili & 55 & $I w b c$ & 50 & & & & & & & & \\
\hline Ipotass & 55 & Isodium & 50 & & & & & & & & \\
\hline |po2po2 & 54 & hidbili & 49 & & & & & & & & \\
\hline Iserum & 54 & po2fio2r & 48 & & & & & & & & \\
\hline po2fio2r & 52 & hbloodp & 44 & & & & & & & & \\
\hline sga & 51 & & & & & & & & & & \\
\hline Iheartr & 48 & & & & & & & & & & \\
\hline seizure & 44 & & & & & & & & & & \\
\hline Itempf & 41 & & & & & & & & & & \\
\hline hpotass & 38 & & & & & & & & & & \\
\hline hsodium & 37 & & & & & & & & & & \\
\hline
\end{tabular}

The variable $b t h w t$ is kept in the model although it is quite low in significance. The occurrence of seizure is removed from this model, which is in accordance with Ennett's model. The variables hpco 2 and lplt were kept in this model although they had been removed in the previous model; both also appear in Ennett's model. Five of the 9 SNAPPE-II variables are kept in the final 9 variable model: lbloodp, lurine, lserum, bthwt and apgar5. Apgar5 appeared low in significance in most iterations but avoided elimination. The variables lheartr, hrespr, and lpo2po2 are consistently high in 
significance in this model and also in the linear model found in 5.4.1. The variables hheartr, hrepsr, hpco 2 and lpotass are also consistently at the top in this model.

Table 5-23: A comparison of the 10 variable model found on linear network compared with the 9 variable model found on a non-linear network for day 3, Ennett's day 1 model and the SNAPPE-II model

\begin{tabular}{llllllll}
\hline Linear Model & \multicolumn{2}{l}{ Non-linear Model } & \multicolumn{2}{l}{ Ennett's Model } & \multicolumn{2}{l}{ SNAPPE-II Model } \\
\hline Iheartr & 100 & hrespr & 100 & po2fio2r & 100 & bthwt & 100 \\
Isodium & 82 & Iplt & 89 & lurine & 72 & apgar5 & 62 \\
hrespr & 73 & Ipotass & 81 & Iserum & 36 & seizure & 43 \\
po2fio2r & 72 & hheartr & 69 & apgar5 & 33 & Itempf & 41 \\
Ibloodp & 65 & guaiac & 69 & Iplt & 29 & Iserum & 25 \\
hhema & 57 & bthwt & 69 & sga & 28 & po2fio2r & 20 \\
Ipo2po2 & 51 & Ipo2po2 & 67 & hsodium & 28 & sga & 15 \\
apgar5 & 47 & Iserum & 65 & hrespr & 24 & lurine & 3 \\
guaiac & 46 & hpco2 & 61 & hpco2 & 22 & Ibloodp & 1 \\
Ipotass & 45 & Ibloodp & 50 & bthwt & 21 & & \\
seizure & 43 & lurine & 50 & Igluc & 20 & & \\
lurine & 34 & apgar5 & 48 & Itempf & 18 & & \\
Iserum & 22 & apnea & 20 & hbloodp & 18 & & \\
\hline
\end{tabular}

Table 5-23 shows a side-by-side comparison of the 13 variable linear model found in section 5.4.1, the 13 variable non-linear model found in this section, Ennett's 13 variable model found on day 1 data and the SNAPPE-II model.

Dr. Walker of CHEO said that the day 3 linear and non-linear models "make good clinical sense". Dr. Walker confirmed that many of the variables in both the linear and non-linear (such as lbloodp, lurine) are associated with sick infants and therefore he agrees that they should be in the model. Dr.Walker also confirmed that birthweight is more significant in the first two days of life, after which even the smallest babies have a $90 \%$ chance of survival, so it makes sense that bthwt and sga were not found to be significant in the models. Dr.Walker confirmed that the respiratory variables such as po2fio2r, lpo2po2 and hpco2 are important variables to physicians, although oi (oxygenation index) is eliminated but is used quite often by physicians to predict outcome [Walker, 2006].

If these models are eventually used to by a real-time prediction system for the clinical environment as is the ultimate goal of this research, the models should be re- 
assessed from time to time to ensure that they have not become obsolete. As NICU technologies continue to rapidly progress, the variables found to be most significant in predicting mortality in future research will not necessarily be the same as those found in this research.

\subsection{Comparing the Models}

The linear model shown in the left-most column of table 5-23 was used to impute variables into the full day 3 database of 13,871 cases. The ANN results of the full, imputed database is shown in table 5-24. Only two structures were recorded in tables 524 and 5-25, since only two fit the selection criteria outline in section 2.2.5. An average sensitivity of $70.5 \%$ was achieved for a non-linear structure with 4 hidden nodes while a specificity of above $91 \%$ was maintained and an area under the ROC was recorded as 86.5. The average sensitivity achieved was significantly higher than that achieved by the SNAPPE-II model and Ennett's day 1 model. The structure that gave the highest average sensitivity was a non-linear structure with 4 hidden nodes. This shows that although a model maybe built using a linear network structure, its performance may be highest on a non-linear network structure.

Table 5-24: ANN performance results for linear 13 variable model for full day 3 database $(\mathrm{N}=13,871)$

\begin{tabular}{|l|l|l|l|}
\hline \multicolumn{2}{|l}{ 0 hidden } & 4 hidden \\
\hline Best test epoch & 676 & 306 \\
\hline \multirow{2}{*}{ Log sensitivity } & Best test & 0.29541 & 0.28374 \\
\cline { 2 - 4 } & Verification average & 0.258486 & 0.320942 \\
\hline \multirow{2}{*}{ Sensitivity (\%) } & Best test & 68.7 & 67.5 \\
\cline { 2 - 4 } & Verification average & 65.0 & 70.5 \\
\hline \multirow{2}{*}{ Specificity (\%) } & Best test & 91.5 & 92.0 \\
\cline { 2 - 4 } & Verification average & 91.3 & 91.5 \\
\hline Classification Rate (CR) (\%) & 90.9 & 91.3 \\
\hline Area under ROC & 85.1 & 86.5 \\
\hline
\end{tabular}

The 13 variables and their weights for the non-linear model (shown in the middle column of table 5-23) was also used to impute the full database of 13,871 cases of day 3 
data. The results of the ANN performance are shown in table 5-25. It was expected that the non-linear model would be able to better capture the non-linearity of the medical data and would therefore perform better. The results of table 5-25 show that its average sensitivity achieved was not as high (65.1\% compared to $70.5 \%$ for the linear model) as the linear model's sensitivity. Also, the non-linear model was able to consistently score very high sensitivities for the best test epoch. The best test sensitivity for the best structures was consistently greater than $71 \%$ which is the highest test sensitivity achieved by any of the models studied (SNAPPE-II, Ennett's and the linear model). Although the average sensitivity was not as high as the linear model's the area under the ROC is about the same for both models ( 86.5 and 86.3). The structure with 3 hidden nodes had an average sensitivity that was very slightly higher than the structure with 7 nodes $(65.1 \%$ vs. $65.0 \%$ ), however its area under the ROC is considerably lower (81.6 vs. 86.3). Therefore, the structure with 7 hidden nodes was taken as the best performing structure.

Table 5-25: ANN performance results for 13 variable non-linear model for full day 3 database $(\mathrm{N}=13,871)$

\begin{tabular}{|l|l|l|l|}
\hline \multicolumn{2}{|l}{3 hidden } & 7 hidden \\
\hline Best test epoch & 333 & 214 \\
\hline \multirow{2}{*}{ Log sensitivity } & Best test & 0.32321 & 0.3205 \\
\cline { 2 - 4 } & Verification average & 0.255664 & 0.253702 \\
\hline \multirow{3}{*}{ Sensitivity (\%) } & Best test & 71.0 & 71.1 \\
\cline { 2 - 4 } & Verification average & 65.1 & 65.0 \\
\hline \multirow{2}{*}{ Specificity (\%) } & Best test & 91.3 & 90.9 \\
\cline { 2 - 4 } & Verification average & 91.0 & 90.8 \\
\hline \multirow{2}{*}{ Classification Rate (CR) (\%) } & 90.8 & 90.3 \\
\hline Area under ROC & $\mathbf{8 1 . 6}$ & 86.3 \\
\hline
\end{tabular}

Table 5-26 is a complete view of the four models applied to the day 3 database. The performance of the best structure of the 0 to $2 n+1$ (where $n$ varied depending on the number of input variables required for the model) structures run on the ANN RFW are shown. Each model was applied on the same number of day 3 cases $(13,871$ cases in total). The initial parameters for each model were the ones that gave the best results and 
are shown in appendix D. The ROC curves of the best performing structure for each model are also shown in appendix $\mathrm{D}$.

The linear model was able to achieve the highest average sensitivity over the ten verification sets $(70.5 \%)$. The next best average sensitivity was of Ennett's model (67.8\%). The non-linear model however, was able to achieve the highest best test sensitivity $(71.1 \%)$. Both the linear and non-linear models were able to achieve a higher area under the ROC than Ennett's model. SNAPPE-II had the lowest performance of all four models.

It was expected that the non-linear model would have achieved the highest average sensitivity, but this was not the case. One explanation is that for the neural network to obtain such a high sensitivity at the best test epoch (71.1\%) it had to memorize some of the data and therefore, overfitting occurred. When it applied what it had learned to the verification sets that consist of a new set of cases, it was not able to obtain as high a performance. The ANN RFW optimizes the test log sensitivity by increasing the test sensitivity to the highest possible. So, it is easy for overfitting to occur if the verification average sensitivity is not also checked to ensure it does not decrease as the best test epoch sensitivity increases.

Table 5-26: A comparison of the best performing ANN structure for the four different mortality prediction models on day 3 data $(\mathrm{N}=13,871)$

\begin{tabular}{|c|c|c|c|c|c|}
\hline \multicolumn{2}{|c|}{ 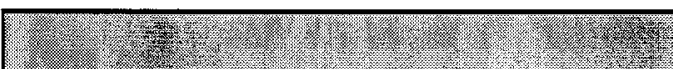 } & SNAPPEII & Ennett's & Linear & Non-linear \\
\hline \multicolumn{2}{|c|}{ Number of input variables $(n)$} & 9 & 13 & 13 & 13 \\
\hline \multicolumn{2}{|c|}{ Structure (number of hidden nodes) } & 2 & 0 & 4 & 7 \\
\hline \multicolumn{2}{|l|}{ Best test epoch } & 338 & 786 & 306 & 214 \\
\hline \multirow{2}{*}{ Log sensitivity } & Best test & 0.2782 & 0.29583 & 0.28374 & 0.3205 \\
\hline & Verification average & 0.15283 & 0.283714 & 0.320942 & 0.253702 \\
\hline \multirow{2}{*}{ Sensitivity $(\%)$} & Best test & 67.4 & 69.2 & 67.5 & 71.1 \\
\hline & Verification average & 52.6 & 67.8 & 70.5 & 65.0 \\
\hline \multirow{2}{*}{ Specificity (\%) } & Best test & 90.9 & 90.4 & 92.0 & 90.9 \\
\hline & Verification average & 90.7 & 90.5 & 91.5 & 90.8 \\
\hline \multicolumn{2}{|c|}{ Classification Rate (CR) $(\%)$} & 90.3 & 89.9 & 91.3 & 90.3 \\
\hline \multicolumn{2}{|c|}{ Area under ROC } & 84.5 & 85.2 & 86.5 & 86.3 \\
\hline
\end{tabular}


From the results in table 5-26, it is apparent that both the linear model and nonlinear models, give better performance when predicting mortality on day 3 NICU data than the SNAPPE-II model and Ennett's model.

\subsubsection{Analysis of the Best Performing Structure}

An interesting observation can be made from table 5-26. The second row of the table shows which structure (i.e. how many hidden nodes) performed the best out of the 0 to $2 n+1$ structures attempted for each model, where $n$ is shown in the first row of the table. For $n=13$ for the linear model, 4 hidden nodes gave the best performance. Similarly for $\mathrm{n}=13$ for the non-linear model, 7 hidden nodes gave the best performance. In section 2.2.4, selecting the network structure was discussed; in particular Masters' method was discussed. According to Masters' theorem for calculating the number of nodes ( hidden $=\sqrt{\text { input } * \text { output }}$ as shown in equation 2-9), using 13 input variables, 3.6 hidden nodes would be required. Master's theorem in the case of the linear, SNAPPE-II and Ennett's models was quite accurate in its estimate of the number of hidden nodes as seen in table 5-26. Running the ANN with the number of nodes obtained from Master's theorem as an upper limit (plus or minus one hidden node) would have been sufficient for these structures. Had this been the case a significant amount of computation time could have been saved, since the ANN RFW takes quite long to run $2 n+1$ structures and the time increases exponentially as the number of hidden nodes increases. Although, the best structure taken for the non-linear model had 7 hidden nodes, the next best performing structure in table 5-35 had 3 hidden nodes. The difference in performance of these two structures was very small. So, again, Master's theorem was accurate in its prediction of hidden nodes.

It should be noted however, that when a smaller number of cases were used $(2,364)$ to find the minimum dataset as shown in table 5-21, the best performing structure had between 11 and 19 hidden nodes. This can be explained by the fact that when the neural network has more cases it requires a smaller number of hidden nodes to learn the trends of the data. With fewer cases, a larger number of hidden nodes are required to be able to find the trends in the data. 
Ennett's model on the other hand, performed best on a 2-layered structure with 0 hidden nodes. Therefore, a safer approach that encompasses all potential best structures, would be to run a structure with 0 hidden nodes to the number of nodes required by Masters' theorem. So, for the four models in table 5-26 running structures from 0 to 4 hidden nodes would have sufficed. This observation will likely have a major impact on work by MIRG if adapted as a reasonable approach for future ANN experiments. 


\subsection{Conclusion}

\subsection{Discussion}

The goal of this research was to develop a mortality model for day 3 that is able to predict mortality better than the conventional models. Although there are numerous neonatal mortality prediction models in the literature none is aimed at predicting mortality using data after the admission day.

In the process of developing a mortality model, the MIRG $\mathrm{kNN}$ case based reasoner tool was tested and verified. It was able to impute values as accurately as the previous CBR tool. It was also discovered that various weight assignations to the variables that were given to the $\mathrm{kNN}$ did not affect its ability to impute missing values. Therefore, linear network weights were used for the most part because they are easier to use and less prone to error.

It was also discovered that running structures with 0 hidden nodes to $2 n+1$ hidden nodes for a dataset of 13,871 cases was futile. Masters' theorem encompassed most of the best performing structures, so future work using large training sets could limit the experiments to those structures and save much computing time.

Mortality models that performed better than the SNAPPE-II model and Ennett's model for day 1 were created by reducing a 2-layered network and a 3-layered network to obtain a minimum dataset.

It was verified that the SNAPPE-II model loses its predictive power as time progresses in the NICU as has been stated in the literature [Meadow et al., 2002; Mueller et al., 2004]. Another important discovery was that the variable bthwt is not a significant predictor of mortality after admission day contrary to conventional belief. Respiratory variables seemed to be quite significant in predicting mortality on day 3 , as were the variables for low blood pressure and low urine output.

Therefore, the goal of developing a mortality model that can predict mortality with a sensitivity of close to $50 \%$ and specificity of at least $90 \%$ was successfully achieved. 


\subsection{Research Contributions}

1) The ability of the $\mathrm{kNN}$ tool to impute missing values with an accuracy similar to the formerly used CBR tool was verified.

2) Imputation using linear (2-layered) network weights was compared to imputation using non-linear (3-layered) network weights and it was found that these different weights of the variables do not impact the accuracy of the imputed values.

3) Two new models were developed for mortality prediction using day 3 data. One using a linear network and one using a non-linear network. MIRG's expert physician verified that the models make good clinical sense.

4) The linear model was able to obtain the highest average sensitivity of the SNAPPE-II model, Ennett's model and the non-linear model. The non-linear model obtained the highest test sensitivity. Both models had an area under the ROC that was higher than the SNAPPE-II and Ennett's models.

5) Masters' theorem for selecting the number of hidden nodes was shown to be a good approach for day 3 data when predicting mortality using a large dataset; this, if applied to future research by MIRG can save a lot of computing time and human resources time.

\subsection{Future Work}

\section{kNN Tool}

1) To examine why the weights of the variables input to the $\mathrm{kNN}$ do not impact the performance of the tool in the imputation of missing values

\section{ANN RFW Tool}

2) To determine why the ANN RFW gives different results for different initial parameter values and determine how to have reproducible results

3) To change the 'best Result Structure' selection criteria so that only structures with a best test epoch that occurs after 100 epochs are selected

4) To change the 'best Result Structure' selection criteria so only structures that give acceptable verification results are selected 
5) To modify the tool so that the stop criterion may be easily changed. For example, so that the network specificity will always be above $98 \%$.

6) To determine why the weights that are extracted using the extended Garson-Goh method from a non-linear (3-layered structure) are often clustered in one weight range and why changing the weight decay constant (wnot) does not seem to affect the weight distribution

7) Try other ANN learning methods such as the Unscented Kalman filtering, the extended Kalman filtering method or Newton-based methods such as the Levenberg-Marquardt method instead of the steepest descent backpropagation method used by the current ANN tool. Compare convergence speeds of the different methods.

\section{Other Areas}

8) To study the effects of confounding variables in the day 3 models such as po2fio2r and lpo2po2

9) To verify the performance of the linear and non-linear day 3 models on new day 3 NICU data by reducing variables one by one and attempting other approaches such as PCA, radial basis function, etc.

10) To study whether physicians are able to make an accurate mortality prediction given the variables defined in the linear and non-linear day 3 models and whether physician prediction using these two models outperforms the ANN or not.

11) To develop a neonatal mortality prediction model for other time periods such as day 10 , day 14 , etc. 


\section{References}

Alberdi, E. Gilhooly, K. Hunter, J. Logie, R. Lyon, A. McIntosh, N. Reiss, J.

"Comptuerisation and decision making in neonatal intensive care: a cognitive engineering investigation”. Journal of Clinical Monitoring and Computing. 2000; 16(2): 85-94.

Ambalavanan, N. Waldemar, AC. "Comparison of the prediction of extremely low birth weight neonatal mortality by regression analysis and by neural networks". Early Human Development. December 2000; 65(2): 123-137.

Apgar, V. "A proposal for a new method of evaluation of the newborn infant". Anesth Analg. 1953; 32: 260-7.

Chittaro, L. Dojat, M. "Using a General Theory of Time and Change in Patient Monitoring: Experiment and Evaluation". Comput. Biol. Med. 1997; 27(5); 435-452.

Civetta, JM. Hudson-Civetta, JA. Nelson, LD. "Evaluation of APACHE II for cost containment and quality assurance". Ann Surg. 1990 September; 212(3): 266-276.

Combi, C. Shahar, Y. "Temporal Reasoning and Temporal Data Maintenance in Medicine: Issues and Challenges”. Comput. Biol. Med. 1997; 27(5): 353-368.

Cotea, C. Jiwani, S. A Case-Based Reasonsing (CBR) System for the Neonatal Intensive Care Unit (NICU). Bachelor of Science in Engineering Thesis. University of Ottawa, Ottawa, ON, Canada, 2003.

Ennett, CM. Imputation of Missing Values by Integrating Artificial Neural Networks and Case Based Reasoning. Doctor of Philosophy in Electrical Engineering Thesis, Carleton University, Ottawa, ON, Canada. 2003. 
Ennett, CM. Frize, M. "Weight-Elimination Neural Networks Applied to Coronary Surgery Mortality Predcition" IEEE Transactions on Information Technology in Biomedicine. 2003; 7(2): 86-92.

Ennett, CM. Frize, M. Scales, N. "Logarithmic sensitivity index as a stopping criterion for neural networks". Proc IEEE-EMBS/BMES , 2002.

Ennett, CM. Frize, M. Walker, CR. Influence of missing values on artificial neural network performance. Medinfo, 2001.

Ennett, CM. Frize, M. "Selective Sampling to Overcome Skewed a priori Probabilities with Neural Networks" Proc AMIA Symposium. 2000: 225-29.

Fausett, L. Fundamentals of Neural Networks. Prentice-Hall: Englewood Cliffs, NJ, 1994.

Fowlie, PW. Tarnow-Mordi, WO. Gould, CR. Strang, D. "Predicting outcome in very low brthwt infants using an objective measure of illness servirty and cranial ultrasound scanning". Arch. Dis. Child. Fetal Neonatal Ed. 1998; 78: 175-178.

Frize et al. "Conceptual Framework of Knowledge Management for Ethical DecisionMaking Suppport in Neonatal Intensive Care" IEEE. 2005; 9(2): 205-215.

Frize and Walker. Personal communication from a document related to a grant application cited by S.Khan, Fall 2003.

Frize et al. "Clinical decision support systems for intensive care units: Using artificial neural networks" Medical Engineering and Physics. 2001; 23: 217-225. 
Frize, M. Ennett, CM. Charette, E. Automated optimization of the performance of artificial neural networks to estimate medical outcomes. Proc 3rd ITAB Conference (Information Technology Applications in Biomedicine) and ITIS (International Telemedical Information Society), 2000 (6 pages).

Frize and Walker "Clinical Decision-Support Systems for Intensive Care Units Using Case-Based Reasoning" Medical Engineering and Physics. 2000; 22: 671-77.

Frize, M. Wang, L. Ennett, CM. Nickerson, B. Solven, FG. Stevenson, M. "New advances and validation of knowledge management tools for critical care using classifier techniques". Proc AMIA Symp, 1998: 553-558.

Frize, M. Solven, FG. Stevenson, M. Nickerson, BG. Buskard, T. Taylor, K. "Computerassisted decision support systems for patient management in an intensive care unit". Medinfo, 1995; 8 Pt 2: 1009-1012.

Frize, M. Taylor, K.B. Nickerson, B.G. Solven, F.G. Borkar, H. (1993)

"A Knowledge-Based System for the Intensive Care Unit", IEEE-EMBS"93, San Diego, Oct. 677-678.

Gray, JE. Richardson, DK. McCormick, MC. Workmann-Daniels, K. Goldmann, DA. "Neonatal therapeutic invertvention scoring system: a therapy-based severity-of-illness index." Pediatrics 1992 Oct; 90(4): 561-7.

Haykin, Simon. Neural Networks: A Comprehensive Foundation. Prentice-Hall, New Jersey, 1999.

Hohavi, R. Provost, F. "Glossary of Terms". Special Issue on Application of Machine Learning and the Knowledge Discovery Process. Machine Learning, 30: 271-274. 
Hornik, K. Stinchcombe, M. White, H. "Multilayer feedforward networks are universal approximators." Neural Networks 1989; 2: 359-366.

International Neonatal Network. "The CRIB (clinical risk index for babies) score: a tool for assessing initial neonatal risk and comparing performance of neonatal intensive care units." Lancet 1993;342:193-8.

Richardson, DK. Gray, JE. McCormick, MC. Workman, K. Goldmann, DA. "Score for Neonatal Acute Physiology: A Physiologic Severity Index for Neonatal Intensive Care". Pediatrics. March 1993; 91(3).

Livingstone, DJ. Manallack, DT. "Statistics using neural networks chance effects," J Med Chem. 1993; 36: 295-97.

Marcin, JP. Pretzlaff, RK. Pollack, MM. Patel, KM. Ruttimann, UE. “Certainty and mortality prediction in critically ill children". J Med Ethics 2004; 30: 304-307.

Masters, T. Practical Neural Network Recipes in C++. San Diego, CA: Academic Press, 1993.

MATLAB built in help disk, release 13.

McGowan, HCE . Stevenson, M. Frize, M. (1996) “The Need for Standardized Reporting of Medical Applications of Artificial Neural Networks", CMBEC, Charlottetown, June 26-29.

Meadow, W. Frain, L. Ren, Y. Lee, G. Soneji, S. Lantos, J. "Serial Assessment of Mortality in the Neonatal Intensive Care Unit by Algorithm and Intuition: Certainty, Uncertainty and Informed Consent." Pediatrics 2002 May; 109; 878-886. 
Meadow, W. Lee, G. Lin, Kathy. Lantos, J. "Changes in Mortality for Extremely Low birth weight infants in the 1990s: Implications for treatment decisions and resource use". Pediatrics, May 2004; 113(5).

Morse, SB. Haywood, JL. Goldenber, RL. Bronstein, J. Nelson, KG. Carlo, WA. "Estimation of Neonatal Outcome and Perinatal Therapy Use." Pediatrics 2000; 105 : 1046-1050.

Mueller, M. Wager, CL. Annibale, DJ. Husley, TC. Knapp, RG. Almeida, JS. "Predicitng Extubation Outcome in Preterm Newborns: A comparison of Neural Networks with Clincal Expertise and Statistical Modeling". Peatrict Research. 2004;.56(1): 11-18.

Olden, J. Jackson, D. “ Illuminating the 'black box': a randomization approach for understanding variable contributions in artificial neural networks," Department of Zoology, University of Toronto, Ecological Modelling, 2002; 154:135-150.

Pan, A. "MIRG ANN Guide". School of Information Technology and Engineering, University of Ottawa. 2002.

Penny, W. Frost, D. “Neural networks in clinical medicine." Med Decis Making 1996;16: 386-398.

Petridou, E. Richardson, DK. Dessypris, N. Malamitsi-Puchner, A. Mantagos, S. Nicolopoulos, D. Papas, C. Salvanos, H. Sevastiadou, S. Sofatzis, J. Trichopoulos, D. "Outcome Prediction in Greek Neonatal Intensive Care Units Using a Score for Neonatal Acute Physiology (SNAP).” Pediatrics 1998 June; 101: 1037-1044

Qi, Lili. Evaluation of an Artificial Neural Network Tool for Neonatal Intensive Care Units. Master in Information and Systems Science Thesis, Department of Systems and Computer Engineering, Carleton University, Ottawa, ON, Canada. 2005. 
Richardson, DK. Corcoran, JD. Escobar, GJ. Lee, SK. "SNAP-II and SNAPPE-II: Simplified newborn illness severity and mortality risk scores". Journal of Pediatrics. 2001;138: 92-100.

Richardson, DK. Gray, JE. Gortmaker, SL. Goldmann, DA. Pursley, DM. McCormick, MC. "Declining Severity Adjusted Mortality: Evidence of Improving Neonatal Intensive Care". Pediatrics, Oct 1998; 102: 893 - 899.

Richardson, DK. Gray, JE. McCormick, MC. Workman, K. Goldmann, DA. "Score for Neonatal Acute Physiology: a physiologic severity index for neonatal intensive care". Pediatrics 1993;91: 617-623.

Rybchynski, D. Design of an Artificial Neural Network Research Framework: Efficient Development of More Effective Prediction Models for Medical Outcomes. Master of Applied Science in electrical Engineering Thesis, School of Information Technology and Engineering, University of Ottawa, Ottawa, ON, Canada. 2005.

Sankaran, K. Chien, L. Walker, R. Seshia, M. Ohlsson, A. Lee, SK and the Canadian Neonatal Network. "Variations in mortality rates among Canadian neonatal intensive care units". CMAJ 2002; 166(2): 173-8.

Scales, N. Mortality prediction in an ICU and coronary surgery database using neural networks. Term project, Department of Systems and Computer Engineering, Carleton University, Dec 2001.

Stevens, SM. Richardson, DK. Gray, JE. Goldmann, DA. McCormick, MC. "Estimating neonatal mortality risk: an analysis of clinicians' judgements." Pediatrics $1994 ; 93(6): 945-950$. 
Walker, CR [MBBS, FRCPC]. Chief Neonatologist, Children's Hospital of Eastern Ontario (CHEO), Ottawa Hospital - General Campus, Ottawa, ON. Personal communication cited by S. Khan, August 1, 2006.

Walker, CR [MBBS, FRCPC]. Chief Neonatologist, Children's Hospital of Eastern Ontario (CHEO), Ottawa Hospital - General Campus, Ottawa, ON. Personal communication cited by C. M. Ennett, May 20, 2003.

Walker, CR. Ennett, CM. Frize, M. "Use of an Artificial Neural Network (ANN) to Estimate Probability of Mortality and Duration of Ventilation in Neonatal Intensive Care Unit (NICU) Patients". MedInfo 2001.

Wilson, A. Gardner, MN. Armstrong, MA. Folck, BF, Escobar, GJ. "Neonatal assisted ventilation: Predictors, frequency and Duration in a Mature Managed Care organization”. Pediatrics 2000; 105; 822-830.

Yang, Lan. Pilot Usability Study of UI Prototype for Collaborative Adaptive Decision Support in Neonatal Intensive Care Unit. Master's in Applied Science Electrical Engineering Thesis, School of Information Technology and Engineering, University of Ottawa, Ottawa, Canada, 2004.

Ye, W. The Study of the Performance of Artificial Neural Networks in the Neonatal Intensive Care Unit. Undergraduate Thesis, Carleton University, ON, Canada. 2000.

Zernikow, B. Holtmannspoetter, K. Michel, E. Pielemeier, W. Hornschuh, F. Westermann, A. Hennecke, K.H. "Artificial neural network for risk assessment in preterm neonates". Arch. Dis. Child. Fetal Neonatal Ed. 1998; 79; 129-134. 


\section{Appendix A - SNAP Variable Listings}

Table A-1. SNAP variable list and scoring system [Richardson et al., 1993]

\begin{tabular}{|c|c|c|c|c|c|}
\hline \multirow[t]{2}{*}{ Variable } & & \multicolumn{4}{|c|}{ Points scored } \\
\hline & & $\mathbf{0}$ & 1 & 3 & 5 \\
\hline \multirow[t]{2}{*}{ Mean blood pressure } & High & $<66$ & $66-80$ & $81-100$ & $>100$ \\
\hline & Low & $>35$ & $30-35$ & $20-29$ & $<\overline{20}$ \\
\hline \multirow[t]{2}{*}{ Heart rate } & High & $<180$ & $180-200$ & $201-250$ & $>250$ \\
\hline & Low & $>100$ & $80-100$ & $40-79$ & $<40$ \\
\hline Respiratory rate & High & $<60$ & $60-100$ & $>100$ & - \\
\hline Temperature $\left({ }^{\circ} \mathrm{F}\right)$ & Low & $>96$ & $95-96$ & $92-94.9$ & $<92$ \\
\hline pO2 & Low & $>65$ & $50-65$ & $30-50$ & $<30$ \\
\hline pO2/FiO2 ratio & Low & $>3.5$ & $2.5-3.5$ & $0.3-2.49$ & $<0.3$ \\
\hline $\mathrm{pCO} 2$ & High & $<50$ & $50-65$ & $66-90$ & $>90$ \\
\hline Oxygenation index & High & $<0.07$ & $0.07-0.2$ & $0.21-0.40$ & $>0.40$ \\
\hline \multirow[t]{2}{*}{ Hematocrit } & High & $<66$ & $66-70$ & $>70$ & - \\
\hline & Low & $>35$ & $30-35$ & $20-29$ & $<20$ \\
\hline White blood count & Low & $>5.0$ & $2.0-5.0$ & $<2.0$ & - \\
\hline $\begin{array}{l}\text { Immature/total neutrophil } \\
\text { ratio }\end{array}$ & High & $<0.21$ & $\geq 0.21$ & - & - \\
\hline Absolute neutrophil count & Low & $>999$ & $500-999$ & $<500$ & - \\
\hline Platelet count & Low & $>100$ & $30-100$ & $0-29$ & - \\
\hline Blood urea nitrogen & High & $<40$ & $40-80$ & $>80$ & \\
\hline Creatinine & High & $<1.2$ & $1.2-2.4$ & $2.5-4.0$ & $>4.0$ \\
\hline Urine output & Low & $>0.9$ & $0.5-0.9$ & $0.1-0.49$ & $<0.1$ \\
\hline $\begin{array}{l}\text { Indirect bilirubin } \\
\text { - bili for birth weight }>2 \mathrm{~kg} \\
\text { - bili/kg for birth weight }<2 \mathrm{~kg}\end{array}$ & High & $\begin{array}{l}<15 \\
<5\end{array}$ & $\begin{array}{c}15-20 \\
5-10 \\
\end{array}$ & $\begin{array}{l}>20 \\
>10\end{array}$ & - \\
\hline Direct bilirubin & High & $<2.0$ & $>2.0$ & - & - \\
\hline \multirow{2}{*}{ Sodium } & High & $<150$ & $150-160$ & $160-180$ & $>180$ \\
\hline & Low & $>130$ & $120-130$ & $<120$ & - \\
\hline \multirow[t]{2}{*}{ Potassium } & High & $<6.6$ & $6.6-7.5$ & $7.6-9.0$ & $>9.0$ \\
\hline & Low & $>2.9$ & $2.0-2.9$ & $<2.0$ & - \\
\hline \multirow[t]{2}{*}{ Total calcium } & High & $<12$ & $>12$ & - & - \\
\hline & Low & $>6.9$ & $5.0-6.9$ & $<5.0$ & - \\
\hline \multirow[t]{2}{*}{ lonized calcium } & High & $<1.4$ & $>1.4$ & - & - \\
\hline & Low & $>1.0$ & $0.8-1.0$ & $<0.8$ & - \\
\hline \multirow[t]{2}{*}{ Glucose } & High & $<150$ & $150-250$ & $>250$ & - \\
\hline & Low & $>40$ & $30-40$ & $<30$ & - \\
\hline \multirow[t]{2}{*}{ Serum bicarbonate } & High & $<33$ & $>33$ & - & - \\
\hline & Low & $>15$ & $11-15$ & $<10$ & - \\
\hline Serum $\mathrm{pH}$ & Low & $>7.30$ & $7.20-7.30$ & $7.10-7.19$ & $<7.10$ \\
\hline Presence of seizures & & None & Single & Multiple & - \\
\hline Presence of apnea & & none & $\begin{array}{l}\text { Response } \\
\text { to stimuli }\end{array}$ & $\begin{array}{l}\text { No } \\
\text { response } \\
\text { to stimuli }\end{array}$ & $\begin{array}{l}\text { Complet } \\
\text { e apnea }\end{array}$ \\
\hline Stool guaiac & & Negative & Positive & - & - \\
\hline
\end{tabular}


* Additional points scored for the Perinatal Extension (SNAPPE) are:

Birth weight $\leq 749 \mathrm{~g}$

30 points

Birth weight 750-999 g

10 points

Apgar $<7$ at 5 minutes

10 points

Small for gestational age $\left(<5^{\text {th }}\right.$ percentile $) \quad 5$ points

Table A-2. SNAP-II variable list and scoring system [Richardson et al. 2001]

\begin{tabular}{llc}
\hline Variable & Range & Points \\
\hline Lowest blood pressure & $\mathrm{MBP}^{\mathrm{a}} 20-29 \mathrm{mmHg}$ & 9 \\
& $\mathrm{MBP} \mathrm{P}^{\mathrm{a}}<20 \mathrm{mmHg}$ & 19 \\
Lowest temperature & $95-96^{\circ} \mathrm{F}$ & 8 \\
& $<95^{\circ} \mathrm{F}$ & 15 \\
Lowest $\mathrm{PO}_{2} / \mathrm{FiO}_{2}$ ratio & $1.0-2.49$ & 5 \\
& $0.3-0.99$ & 16 \\
& $<0.3$ & 28 \\
Lowest serum pH & $7.10-7.19$ & 7 \\
& $<7.10$ & 16 \\
Multiple seizures & $>1$ & 19 \\
Lowest urine output & $0.1-0.9 \mathrm{~mL} / \mathrm{kg} / \mathrm{h}$ & 5 \\
& $<0.1 \mathrm{~mL} / \mathrm{kg} / \mathrm{h}$ & 18 \\
Birth weight & $750-999 \mathrm{~g}$ & 10 \\
& $<750 \mathrm{~g}$ & 17 \\
Small for gestational age & $<3^{\text {do }} \mathrm{percentile}$ & 12 \\
Apgar score at 5 minutes & $<7$ & 18 \\
\hline
\end{tabular}

${ }^{\mathrm{a}} \mathrm{MBP}=$ mean arterial blood pressure

Table A-3. Apgar scoring system [Ennett, 2003]

\begin{tabular}{llll} 
& \multicolumn{1}{c}{$\mathbf{0}$} & \multicolumn{1}{c}{$\mathbf{1}$} & \multicolumn{1}{c}{$\mathbf{2}$} \\
\hline Appearance & Pale & Blue & Pink \\
Pulse & Absent & $<100$ & $>100$ \\
Grimace & Absent & Grimace & Cry active \\
Activity & Limp & Some tone & Active \\
Respiration & Absent & Irregular & Regular and cry \\
\hline
\end{tabular}




\section{Appendix B - Variable Description}

Table B-1: SPSS variable name and transformation formulae for SNAP variables [Ennett, 2003]

\begin{tabular}{|c|c|c|}
\hline Parameter & Units & Variable name and transformation (if necessary) \\
\hline $\begin{array}{l}\text { Highest mean blood } \\
\text { pressure }\end{array}$ & $\mathrm{mmHg}$ & Hbloodp \\
\hline $\begin{array}{l}\text { Lowest mean blood } \\
\text { pressure }\end{array}$ & $\mathrm{mmHg}$ & Lbloodp \\
\hline Highest heart rate & Beats/min & Hheartr \\
\hline Lowest heart rate & Beats/min & Lheartr \\
\hline Highest respiratory rate & Breaths/min & Hrespr \\
\hline Lowest temperature & ${ }^{\circ} \mathrm{F}$ & Ltempf $=\mid$ temp ${ }^{\star} 9 / 5+32$ \\
\hline Lowest pO2 & $\%$ & Lpo2po2 \\
\hline Lowest $\mathrm{pO} 2 / \mathrm{FiO} 2$ ratio & $\% / \%$ & $\begin{array}{l}\text { Po2fio2r=MIN(lpo2po2/lpo2fio2, mawppo2/mawpfio2, } \\
\text { fio2po2/fio2fio2) }\end{array}$ \\
\hline Highest pCO2 & $\%$ & Hpco2 \\
\hline Highest oxygenation index & $\% / \%$ & $\begin{array}{l}\text { Oi=MAX(lpo2mawp/lpo2po2*Ipo2fio2, } \\
\text { mawpmawp/mawppo2*mawpfio2, } \\
\text { fio2mawp/fio2po2*fio2fio2)/100 }\end{array}$ \\
\hline Highest hematocrit & $\%$ & Hhema \\
\hline Lowest hematocrit & $\%$ & Lhema \\
\hline Lowest white blood count & $10^{3}$ cells & Lwbc=MIN (cbc1wbc, cbc2wbc, cbc3wbc) \\
\hline $\begin{array}{l}\text { Highest immature/total } \\
\text { neutrophil ratio }\end{array}$ & $10^{3}$ cells & $\begin{array}{l}\text { Hitnrat=MAX (cbc1ban/(cbc1ban+cbc1pol), } \\
\text { cbc2ban/(cbc2ban+cbc2pol), } \\
\text { cbc3ban/(cbc3ban+cbc3pol)) }\end{array}$ \\
\hline $\begin{array}{l}\text { Lowest absolute neutrophil } \\
\text { count }\end{array}$ & $10^{3}$ cells & $\begin{array}{l}\text { Lanc=MIN (cbc1wbc* }(c b c 1 p o l+c b c 1 b a n), \\
c b c 2 w_{b c}^{*}(c b c 2 p o l+c b c 2 b a n) \\
\left.c b c 3 w_{b c}^{*}(c b c 3 p o l+c b c 3 b a n)\right)\end{array}$ \\
\hline Lowest platelet count & $10^{3}$ cells & Lplt=MIN(cbc1plt, cbc2plt, cbc3plt) \\
\hline $\begin{array}{l}\text { Highest blood urea } \\
\text { nitrogen }\end{array}$ & & Hbun \\
\hline Highest creatinine & $\mathrm{mg} / \mathrm{dl}$ & Hcreat \\
\hline Lowest urine output & $\mathrm{cc} / \mathrm{kg} / 24 \mathrm{hr}$ & Lurine $=$ urine/bthwt/24*1000 \\
\hline $\begin{array}{l}\text { Highest indirect bilirubin } \\
\text { - bili for } B W>2 \mathrm{~kg} \\
\text { - bili/kg for } B W<2 \mathrm{~kg}\end{array}$ & & Hidbili \\
\hline Highest direct bilirubin & & Hdbili \\
\hline Highest sodium & $\mathrm{mmol} / \mathrm{L}$ & Hsodium \\
\hline Lowest sodium & $\mathrm{mmol} / \mathrm{L}$ & Lsodium \\
\hline Highest potassium & $\mathrm{mmol} / \mathrm{L}$ & Hpotass \\
\hline Lowest potassium & $\mathrm{mmol} / \mathrm{L}$ & Lpotass \\
\hline Highest total calcium & & Hcalctot \\
\hline Lowest total calcium & & Lcalctot \\
\hline Highest ionized calcium & & Hcalcion \\
\hline Lowest ionized calcium & & Lcalcion \\
\hline Highest glucose & & Hgluc \\
\hline
\end{tabular}




\begin{tabular}{|l|l|l|}
\hline Lowest glucose & lgluc \\
\hline Highest serum bicarbonate & & Hhco3 \\
\hline Lowest serum bicarbonate & & Lhco3 \\
\hline Lowest serum pH & & Lserum \\
\hline Presence of seizures & & Seizure \\
\hline Presence of apnea & & Apnea \\
\hline Stool guaiac & Guaiac \\
\hline
\end{tabular}




\section{Appendix C - Variable Reduction Results}

Table C-1: Initial parameters of ANN used in model reduction of linear and non-linear network structures

\begin{tabular}{ll}
\hline Parameter & Initial value \\
\hline Weight-elimination & Yes \\
Error ratio & 1.005 \\
Lambda & $5 \mathrm{e}-005$ \\
Lambda decrement & 0.999 \\
Lambda increment & 1.001 \\
Learning rate & 0.0008 \\
Learning rate decrement & 0.9032 \\
Learning rate increment & 1.004 \\
Momentum & 0.99 \\
Weight scale factor & 0.01 \\
Float value & 0 \\
\hline
\end{tabular}

Table C-2: ANN RFW parameters of best test run for linear tests during variable reduction step

\begin{tabular}{|l|l|l|l|l|l|l|}
\hline Parameter & $\begin{array}{l}\mathbf{2 8} \\
\text { variables }\end{array}$ & $\begin{array}{l}\mathbf{2 2} \\
\text { variables }\end{array}$ & $\begin{array}{l}\mathbf{1 6} \\
\text { variables }\end{array}$ & $\begin{array}{l}\mathbf{1 5} \\
\text { variables }\end{array}$ & $\begin{array}{l}\mathbf{1 4} \\
\text { variables }\end{array}$ & $\begin{array}{l}\mathbf{1 3} \\
\text { variables }\end{array}$ \\
\hline Best test epoch & 229 & 388 & 228 & 695 & 573 & 718 \\
\hline Error ratio & 1.005 & 1.005 & 1.005 & 1.0372 & 1.005 & 1.005 \\
\hline Lambda & $5 \mathrm{e}-005$ & $5 \mathrm{e}-005$ & $7.68 \mathrm{e}-005$ & $2.69 \mathrm{e}-005$ & $8.88 \mathrm{e}-005$ & $5 \mathrm{e}-005$ \\
\hline Lambda decrement & 0.3126 & 0.999 & 0.2787 & 0.999 & 0.999 & 0.999 \\
\hline Lambda increment & 1.0079 & 1.001 & 1.0623 & 1.0035 & 1.001 & 1.001 \\
\hline Learning rate & 0.0025351 & 0.0001376 & $5 \mathrm{e}-005$ & $5.13 \mathrm{e}-005$ & 0.003157 & 0.0025375 \\
\hline Learning rate decrement & 0.9375 & 0.9032 & 1.2498 & 0.7945 & 0.9032 & 0.9032 \\
\hline Learning rate increment & 1.004 & 1.004 & 1.004 & 1.004 & 1.004 & 1.004 \\
\hline Momentum & 0.99 & 0.99 & 0.99 & 0.99 & 0.999 & 0.999 \\
\hline Weight scale factor & 0.01 & 0.001 & 0.0011 & 0.00017 & 0.00017 & 0.00017 \\
\hline
\end{tabular}


Table C-3: Complete training, test, and verification set results of 28 variable linear test

\begin{tabular}{|l|l|l|l|l|l|l|l|l|l}
\hline & $\begin{array}{l}\text { Log } \\
\text { Sensitivity } \\
\text { Index }\end{array}$ & Sensitivity & Specificity & CR & ASE & bayes_MDC & AprioriNeg & AprioriPos & ROC \\
\hline 1 & 0.10814 & 0.45106 & 0.94049 & 84.2687 & 0.49272 & 83.9286 & 80.017 & 19.983 & 0.77237 \\
\hline 2 & 0.05394 & 0.33871 & 0.90631 & 84.2676 & 0.47831 & 84.0868 & 88.7884 & 11.2116 & 0.7183 \\
\hline 3 & 0.11418 & 0.47222 & 0.90409 & 86.4277 & 0.43899 & 91.9334 & 90.781 & 9.219 & 0.75937 \\
\hline 4 & 0.19632 & 0.58904 & 0.9096 & 87.9641 & 0.38557 & 90.3969 & 90.653 & 9.347 & 0.796 \\
\hline 5 & 0.14974 & 0.52381 & 0.92061 & 88.8604 & 0.36331 & 93.3419 & 91.9334 & 8.0666 & 0.79332 \\
\hline 6 & 0.098311 & 0.4375 & 0.92329 & 88.3483 & 0.39153 & 90.781 & 91.8054 & 8.1946 & 0.74794 \\
\hline 7 & 0.090772 & 0.42857 & 0.90057 & 85.4033 & 0.4613 & 91.2932 & 90.1408 & 9.8592 & 0.73307 \\
\hline 8 & 0.074778 & 0.39024 & 0.91416 & 85.9155 & 0.43155 & 88.2202 & 89.5006 & 10.4994 & 0.79216 \\
\hline 9 & 0.117 & 0.47368 & 0.91575 & 88.3483 & 0.38002 & 93.2138 & 92.7017 & 7.2983 & 0.78121 \\
\hline 10 & 0.081716 & 0.4058 & 0.91433 & 86.9398 & 0.40446 & 90.781 & 91.1652 & 8.8348 & 0.81822 \\
\hline 11 & 0.14918 & 0.52308 & 0.92039 & 88.7324 & 0.38592 & 92.9577 & 91.6773 & 8.3227 & 0.81703 \\
\hline 12 & 0.069554 & 0.375 & 0.9269 & 88.7324 & 0.36931 & 91.9334 & 92.8297 & 7.1703 & 0.74158 \\
\hline Avg & 0.114155 & 0.461894 & 0.914969 & & & & & & 0.77799 \\
\hline
\end{tabular}

* where set 1 is training set, set 2 is test set and sets 3 through 12 are verification sets

Table C-4: Complete training, test and verification set results of 22 variable linear test

\begin{tabular}{|r|r|r|r|r|r|r|r|r|r}
\hline \multicolumn{1}{|l|}{$\begin{array}{l}\text { Set } \\
\text { Sensitivity } \\
\text { Index }\end{array}$} & Sensitivity & Specificity & CR & \multicolumn{1}{l|}{ ASE } & bayes MDC & AprioriNeg & AprioriPos & \multicolumn{1}{l}{ ROC } \\
\hline 1 & 0.056113 & 0.34043 & 0.92774 & 81.0374 & 0.58923 & 83.9286 & 80.017 & 19.983 & 0.73989 \\
\hline 2 & 0.10417 & 0.45161 & 0.91242 & 86.0759 & 0.42594 & 84.6293 & 88.7884 & 11.2116 & 0.75486 \\
\hline 3 & 0.067694 & 0.375 & 0.90691 & 85.7875 & 0.4324 & 91.1652 & 90.781 & 9.219 & 0.7514 \\
\hline 4 & 0.11054 & 0.46575 & 0.90395 & 86.2996 & 0.43911 & 89.8848 & 90.653 & 9.347 & 0.78935 \\
\hline 5 & 0.13994 & 0.50794 & 0.92479 & 89.1165 & 0.34442 & 92.9577 & 91.9334 & 8.0666 & 0.82481 \\
\hline 6 & 0.056687 & 0.34375 & 0.91911 & 87.1959 & 0.41364 & 90.0128 & 91.8054 & 8.1946 & 0.77261 \\
\hline 7 & 0.074566 & 0.38961 & 0.91477 & 86.2996 & 0.4572 & 91.5493 & 90.1408 & 9.8592 & 0.72493 \\
\hline 8 & 0.059066 & 0.35366 & 0.90272 & 84.507 & 0.48109 & 88.6044 & 89.5006 & 10.4994 & 0.75109 \\
\hline 9 & 0.088721 & 0.42105 & 0.91298 & 87.7081 & 0.40358 & 93.2138 & 92.7017 & 7.2983 & 0.73173 \\
\hline 10 & 0.082035 & 0.4058 & 0.91713 & 87.1959 & 0.40359 & 90.9091 & 91.1652 & 8.8348 & 0.79355 \\
\hline 11 & 0.092245 & 0.43077 & 0.90363 & 86.4277 & 0.41452 & 91.6773 & 91.6773 & 8.3227 & 0.74459 \\
\hline 12 & 0.060878 & 0.35714 & 0.90897 & 86.9398 & 0.42511 & 92.1895 & 92.8297 & 7.1703 & 0.70585 \\
\hline Avg & 0.083237 & 0.405047 & 0.911496 & & & & & & 0.758991 \\
\hline
\end{tabular}


Table C-5: Complete training, test and verification set results of 16 variable linear test

\begin{tabular}{|c|c|c|c|c|c|c|c|c|c|}
\hline Set & $\begin{array}{l}\text { Log } \\
\text { Sensitivity } \\
\text { Index }\end{array}$ & Sensitivity & Specificity & CR & ASE & bayes_MDC & AprioriNeg & AprioriPos & ROC \\
\hline 1 & 0.053941 & 0.33191 & 0.94049 & 81.8878 & 0.5128 & 83.1633 & 80.017 & 19.983 & 0.78474 \\
\hline 2 & 0.048058 & 0.32258 & 0.9002 & 83.5443 & 0.45338 & 84.4485 & 88.7884 & 11.2116 & 0.75264 \\
\hline 3 & 0.091089 & 0.43056 & 0.89563 & 85.2753 & 0.42352 & 91.6773 & 90.781 & 9.219 & 0.78767 \\
\hline 4 & 0.15474 & 0.53425 & 0.91102 & 87.58 & 0.39366 & 89.8848 & 90.653 & 9.347 & 0.80772 \\
\hline 5 & 0.15924 & 0.53968 & 0.91365 & 88.3483 & 0.35521 & 93.2138 & 91.9334 & 8.0666 & 0.848 \\
\hline 6 & 0.067145 & 0.375 & 0.90098 & 85.7875 & 0.39788 & 90.525 & 91.8054 & 8.1946 & 0.78864 \\
\hline 7 & 0.048035 & 0.32468 & 0.8892 & 83.3547 & 0.45306 & 90.781 & 90.1408 & 9.8592 & 0.7477 \\
\hline 8 & 0.05486 & 0.34146 & 0.90558 & 84.6351 & 0.43067 & 89.3726 & 89.5006 & 10.4994 & 0.79239 \\
\hline 9 & 0.072135 & 0.38596 & 0.90608 & 86.8118 & 0.38044 & 93.0858 & 92.7017 & 7.2983 & 0.79419 \\
\hline 10 & 0.063077 & 0.36232 & 0.91152 & 86.2996 & 0.39053 & 91.4213 & 91.1652 & 8.8348 & 0.83675 \\
\hline 11 & 0.10795 & 0.46154 & 0.90223 & 86.5557 & 0.39656 & 91.4213 & 91.6773 & 8.3227 & 0.80026 \\
\hline 12 & 0.048237 & 0.32143 & 0.90897 & 86.6837 & 0.37725 & 92.7017 & 92.8297 & 7.1703 & 0.76546 \\
\hline Avg & 0.086651 & 0.407688 & 0.904486 & & & & & & 0.796878 \\
\hline
\end{tabular}

Table C-6: Complete training, test and verification set results for 15 variable linear test

\begin{tabular}{|c|c|c|c|c|c|c|c|c|c|}
\hline Set & $\begin{array}{l}\text { Log } \\
\text { Sensitivity } \\
\text { Index }\end{array}$ & Sensitivity & Specificity & CR & ASE & bayes_MDC & AprioriNeg & AprioriPos & ROC \\
\hline 1 & 0.08868 & 0.42128 & 0.9118 & 81.3776 & 0.64125 & $8 \overline{3.5884}$ & 80.017 & 19.983 & 0.71059 \\
\hline 2 & 0.094944 & 0.43548 & 0.90631 & 85.3526 & 0.50123 & 84.6293 & 88.7884 & 11.2116 & 0.7139 \\
\hline 3 & 0.12273 & 0.48611 & 0.90691 & 86.8118 & 0.42699 & 91.6773 & 90.781 & 9.219 & 0.7408 \\
\hline 4 & 0.14534 & 0.52055 & 0.91102 & 87.452 & 0.42129 & 89.7567 & 90.653 & 9.347 & 0.74127 \\
\hline 5 & 0.10197 & 0.44444 & 0.9234 & 88.4763 & 0.37796 & 93.2138 & 91.9334 & 8.0666 & 0.76771 \\
\hline 6 & 0.068048 & 0.375 & 0.91074 & 86.6837 & 0.44647 & 90.525 & 91.8054 & 8.1946 & 0.69431 \\
\hline 7 & 0.091697 & 0.42857 & 0.90767 & 86.0435 & 0.45958 & 90.781 & 90.1408 & 9.8592 & 0.69417 \\
\hline 8 & 0.079535 & 0.40244 & 0.90844 & 85.5314 & 0.4673 & 89.3726 & 89.5006 & 10.4994 & 0.70374 \\
\hline 9 & 0.081453 & 0.40351 & 0.92127 & 88.3483 & 0.38155 & 93.0858 & 92.7017 & 7.2983 & 0.69995 \\
\hline 10 & 0.12167 & 0.47826 & 0.92697 & 88.7324 & 0.39943 & 91.4213 & 91.1652 & 8.8348 & 0.74851 \\
\hline 11 & 0.10951 & 0.46154 & 0.91201 & 87.452 & 0.40795 & 91.5493 & 91.6773 & 8.3227 & 0.72107 \\
\hline 12 & 0.060313 & 0.35714 & 0.90207 & 86.2996 & 0.44018 & 92.7017 & 92.8297 & 7.1703 & 0.70563 \\
\hline Avg & 0.098227 & 0.435756 & 0.91305 & & & & & & 0.721716 \\
\hline
\end{tabular}


Table C-7: Complete training, test and verification set results for 14 variable linear test

\begin{tabular}{|r|r|r|r|r|r|r|r|r|r}
\hline \multicolumn{1}{|r|}{$\begin{array}{l}\text { Set } \\
\text { Sensitivity } \\
\text { Index }\end{array}$} & Sensitivity & Specificity & \multicolumn{1}{l|}{ CR } & \multicolumn{1}{l|}{ ASE } & bayes_MDC & AprioriNeg & AprioriPos & ROC \\
\hline 1 & 0.085018 & 0.40851 & 0.93199 & 82.7381 & 0.60776 & 83.7585 & 80.017 & 19.983 & 0.71692 \\
\hline 2 & 0.087332 & 0.41935 & 0.90835 & 85.3526 & 0.4929 & 84.6293 & 88.7884 & 11.2116 & 0.7524 \\
\hline 3 & 0.09332 & 0.43056 & 0.91255 & 86.8118 & 0.44974 & 91.2932 & 90.781 & 9.219 & 0.73894 \\
\hline 4 & 0.096846 & 0.43836 & 0.9096 & 86.5557 & 0.45053 & 89.6287 & 90.653 & 9.347 & 0.75522 \\
\hline 5 & 0.11932 & 0.47619 & 0.92061 & 88.4763 & 0.39421 & 92.9577 & 91.9334 & 8.0666 & 0.76719 \\
\hline 6 & 0.062342 & 0.35938 & 0.91632 & 87.0679 & 0.41774 & 90.2689 & 91.8054 & 8.1946 & 0.75802 \\
\hline 7 & 0.064482 & 0.36364 & 0.92188 & 86.6837 & 0.46255 & 90.781 & 90.1408 & 9.8592 & 0.69172 \\
\hline 8 & 0.05121 & 0.32927 & 0.91416 & 85.2753 & 0.50478 & 89.2446 & 89.5006 & 10.4994 & 0.70172 \\
\hline 9 & 0.089066 & 0.42105 & 0.91575 & 87.9641 & 0.40282 & 92.8297 & 92.7017 & 7.2983 & 0.7053 \\
\hline 10 & 0.097798 & 0.43478 & 0.92978 & 88.6044 & 0.38664 & 91.1652 & 91.1652 & 8.8348 & 0.77433 \\
\hline 11 & 0.053561 & 0.33846 & 0.90223 & 85.5314 & 0.44752 & 91.5493 & 91.6773 & 8.3227 & 0.73704 \\
\hline 12 & 0.048412 & 0.32143 & 0.91172 & 86.9398 & 0.44399 & 92.7017 & 92.8297 & 7.1703 & 0.62064 \\
\hline Avg & 0.077636 & 0.391312 & 0.91546 & & & & & & 0.725012 \\
\hline
\end{tabular}

Table C-8: Complete training, test and verification set results for 13 variable linear test

\begin{tabular}{|r|r|r|r|r|r|r|r|r|r}
\hline \multicolumn{1}{r|}{$\begin{array}{l}\text { Set } \\
\text { Seg }\end{array}$} & $\begin{array}{l}\text { Sensitivity } \\
\text { Index }\end{array}$ & Sensitivity & Specificity & CR & ASE & bayes MDC & AprioriNeg & AprioriPos & ROC \\
\hline 1 & 0.06951 & 0.37447 & 0.9288 & 81.8027 & 0.54304 & 83.5034 & 80.017 & 19.983 & 0.76816 \\
\hline 2 & 0.10417 & 0.45161 & 0.91242 & 86.0759 & 0.44358 & 86.4376 & 88.7884 & 11.2116 & 0.77209 \\
\hline 3 & 0.092201 & 0.43056 & 0.90409 & 86.0435 & 0.45072 & 91.0371 & 90.781 & 9.219 & 0.77985 \\
\hline 4 & 0.15406 & 0.53425 & 0.90819 & 87.3239 & 0.42211 & 89.7567 & 90.653 & 9.347 & 0.79967 \\
\hline 5 & 0.093762 & 0.42857 & 0.9234 & 88.3483 & 0.37677 & 92.9577 & 91.9334 & 8.0666 & 0.82687 \\
\hline 6 & 0.050684 & 0.32813 & 0.91213 & 86.4277 & 0.4428 & 90.2689 & 91.8054 & 8.1946 & 0.75108 \\
\hline 7 & 0.054585 & 0.33766 & 0.92045 & 86.2996 & 0.4418 & 91.1652 & 90.1408 & 9.8592 & 0.75381 \\
\hline 8 & 0.043259 & 0.30488 & 0.91416 & 85.0192 & 0.44387 & 89.5006 & 89.5006 & 10.4994 & 0.8118 \\
\hline 9 & 0.065604 & 0.36842 & 0.91298 & 87.3239 & 0.3885 & 92.8297 & 92.7017 & 7.2983 & 0.80824 \\
\hline 10 & 0.069125 & 0.37681 & 0.91433 & 86.6837 & 0.41188 & 91.1652 & 91.1652 & 8.8348 & 0.8195 \\
\hline 11 & 0.08573 & 0.41538 & 0.91061 & 86.9398 & 0.4192 & 91.2932 & 91.6773 & 8.3227 & 0.78969 \\
\hline 12 & 0.048324 & 0.32143 & 0.91034 & 86.8118 & 0.41699 & 92.5736 & 92.8297 & 7.1703 & 0.76459 \\
\hline Avg & 0.075733 & 0.384609 & 0.913068 & & & & & & 0.79051 \\
\hline
\end{tabular}


Table C-9: ANN RFW parameters of best test run for non-linear tests during variable reduction step

\begin{tabular}{|l|l|l|l|l|l|l|}
\hline Parameter & $\begin{array}{l}28 \\
\text { variables }\end{array}$ & $\begin{array}{l}22 \\
\text { variables }\end{array}$ & $\begin{array}{l}16 \\
\text { variables }\end{array}$ & $\begin{array}{l}15 \\
\text { variables }\end{array}$ & $\begin{array}{l}14 \\
\text { variables }\end{array}$ & $\begin{array}{l}13 \\
\text { variables }\end{array}$ \\
\hline Best test epoch & 168 & 254 & 796 & 142 & 280 & 1787 \\
\hline Error ratio & 1.005 & 1.005 & 1.0118 & 1.005 & 1.005 & 1.005 \\
\hline Lambda & $7.47 \mathrm{e}-005$ & $5 \mathrm{e}-005$ & $3.82 \mathrm{e}-005$ & $5.79 \mathrm{e}-005$ & $7.19 \mathrm{e}-005$ & $5 \mathrm{e}-005$ \\
\hline Lambda decrement & 0.999 & 0.999 & 0.999 & 0.999 & 0.999 & 0.999 \\
\hline Lambda increment & 1.001 & 1.001 & 1.001 & 1.001 & 1.0079 & 1.001 \\
\hline Learning rate & 0.0005359 & 0.0012938 & 0.0016047 & $5.19 \mathrm{e}-005$ & 0.0003622 & $5 \mathrm{e}-005$ \\
\hline Learning rate decrement & 0.9032 & 0.9032 & 0.9371 & 0.9032 & 0.75 & 0.9032 \\
\hline Learning rate increment & 1.2032 & 1.004 & 1.1875 & 1.1016 & 1.004 & 1.0002 \\
\hline Momentum & 0.812 & 0.99 & 0.99 & 0.99 & 0.99 & 0.986 \\
\hline Weight scale factor & 0.01 & 0.01 & 0.0001 & 0.01 & 0.01 & 0.01 \\
\hline
\end{tabular}

Table C-10: Complete training, test, and verification set results of 28 variable non-linear test

\begin{tabular}{|r|r|r|r|r|r|r|r|r|r}
\hline \multicolumn{1}{|l|}{$\begin{array}{l}\text { Set } \\
\text { Sensitivity }\end{array}$} & $\begin{array}{l}\text { lndex } \\
\text { Indensitivity }\end{array}$ & Specificity & CR & ASE & bayes_MDC & AprioriNeg & AprioriPos & ROC \\
\hline 1 & 0.14453 & 0.51489 & 0.92455 & 84.2687 & 0.44211 & 83.9286 & 80.017 & 19.983 & 0.83655 \\
\hline 2 & 0.08658 & 0.41935 & 0.90224 & 84.8101 & 0.45263 & 84.0868 & 88.7884 & 11.2116 & 0.78252 \\
\hline 3 & 0.099185 & 0.44444 & 0.90409 & 86.1716 & 0.41846 & 91.9334 & 90.781 & 9.219 & 0.76421 \\
\hline 4 & 0.14502 & 0.52055 & 0.9096 & 87.3239 & 0.39298 & 90.3969 & 90.653 & 9.347 & 0.78517 \\
\hline 5 & 0.14974 & 0.52381 & 0.92061 & 88.8604 & 0.35361 & 93.3419 & 91.9334 & 8.0666 & 0.81644 \\
\hline 6 & 0.12265 & 0.48438 & 0.91213 & 87.7081 & 0.39035 & 90.781 & 91.8054 & 8.1946 & 0.76292 \\
\hline 7 & 0.073551 & 0.38961 & 0.90483 & 85.4033 & 0.44361 & 91.2932 & 90.1408 & 9.8592 & 0.74097 \\
\hline 8 & 0.084262 & 0.41463 & 0.90129 & 85.0192 & 0.4319 & 88.2202 & 89.5006 & 10.4994 & 0.78835 \\
\hline 9 & 0.097247 & 0.4386 & 0.9116 & 87.7081 & 0.3776 & 93.2138 & 92.7017 & 7.2983 & 0.78744 \\
\hline 10 & 0.094156 & 0.43478 & 0.90309 & 86.1716 & 0.38233 & 90.781 & 91.1652 & 8.8348 & 0.8085 \\
\hline 11 & 0.15727 & 0.53846 & 0.90922 & 87.8361 & 0.37653 & 92.9577 & 91.6773 & 8.3227 & 0.80882 \\
\hline 12 & 0.047887 & 0.32143 & 0.90345 & 86.1716 & 0.38861 & 91.9334 & 92.8297 & 7.1703 & 0.73909 \\
\hline Avg & 0.107097 & 0.451069 & 0.907991 & & & & & & 0.780191 \\
\hline
\end{tabular}


Table C-11: Complete training, test and verification set results of 22 variable non-linear test

\begin{tabular}{|r|r|r|r|r|r|r|r|r|r}
\hline \multicolumn{1}{|l|l}{$\begin{array}{l}\text { Leg } \\
\text { Sensitivity } \\
\text { Index }\end{array}$} & Sensitivity & Specificity & CR & ASE & bayes MDC & AprioriNeg & AprioriPos & ROC \\
\hline 1 & 0.1579 & 0.52766 & 0.94368 & 86.0544 & 0.44949 & 82.6531 & 80.017 & 19.983 & 0.82366 \\
\hline 2 & 0.080332 & 0.40323 & 0.91242 & 85.5335 & 0.44044 & 84.2676 & 88.7884 & 11.2116 & 0.7693 \\
\hline 3 & 0.12093 & 0.48611 & 0.89704 & 85.9155 & 0.41529 & 92.0615 & 90.781 & 9.219 & 0.74826 \\
\hline 4 & 0.1653 & 0.54795 & 0.91384 & 87.9641 & 0.38619 & 91.0371 & 90.653 & 9.347 & 0.78397 \\
\hline 5 & 0.14974 & 0.52381 & 0.92061 & 88.8604 & 0.36591 & 92.9577 & 91.9334 & 8.0666 & 0.7662 \\
\hline 6 & 0.106 & 0.45313 & 0.91911 & 88.0922 & 0.36658 & 91.5493 & 91.8054 & 8.1946 & 0.75874 \\
\hline 7 & 0.067231 & 0.37662 & 0.89489 & 84.379 & 0.45686 & 91.2932 & 90.1408 & 9.8592 & 0.71958 \\
\hline 8 & 0.063569 & 0.36585 & 0.90129 & 84.507 & 0.4554 & 88.9885 & 89.5006 & 10.4994 & 0.73878 \\
\hline 9 & 0.065237 & 0.36842 & 0.90884 & 86.9398 & 0.3873 & 92.3175 & 92.7017 & 7.2983 & 0.7497 \\
\hline 10 & 0.10279 & 0.44928 & 0.91152 & 87.0679 & 0.39386 & 90.525 & 91.1652 & 8.8348 & 0.78671 \\
\hline 11 & 0.11848 & 0.47692 & 0.91341 & 87.7081 & 0.3885 & 92.4456 & 91.6773 & 8.3227 & 0.78421 \\
\hline 12 & 0.06814 & 0.375 & 0.91172 & 87.3239 & 0.38746 & 92.7017 & 92.8297 & 7.1703 & 0.70916 \\
\hline Avg & 0.102742 & 0.442309 & 0.909227 & & & & & & 0.754531 \\
\hline
\end{tabular}

Table C-12: Complete training, test and verification set results of 16 variable non-linear test

\begin{tabular}{|c|c|c|c|c|c|c|c|c|c|}
\hline Set & $\begin{array}{l}\text { Log } \\
\text { Sensitivity } \\
\text { Index } \\
\end{array}$ & Sensitivity & Specificity & CR & ASE & bayes_MDC & AprioriNeg & AprioriPos & ROC \\
\hline 1 & 0.27212 & 0.65532 & 0.93943 & 88.2653 & 0.38383 & 83.0782 & 80.017 & 19.983 & 0.86079 \\
\hline 2 & 0.11136 & 0.46774 & 0.90224 & 85.3526 & 0.46991 & 85.1718 & 88.7884 & 11.2116 & 0.74192 \\
\hline 3 & 0.11252 & 0.47222 & 0.89422 & 85.5314 & 0.46536 & 90.781 & 90.781 & 9.219 & 0.72626 \\
\hline 4 & 0.12155 & 0.47945 & 0.92232 & 88.0922 & 0.42573 & 90.3969 & 90.653 & 9.347 & 0.75189 \\
\hline 5 & 0.13731 & 0.50794 & 0.91226 & 87.9641 & 0.38619 & 92.7017 & 91.9334 & 8.0666 & 0.79445 \\
\hline 6 & 0.089361 & 0.42188 & 0.91492 & 87.452 & 0.39479 & 90.9091 & 91.8054 & 8.1946 & 0.76873 \\
\hline 7 & 0.097211 & 0.44156 & 0.90057 & 85.5314 & 0.45619 & 92.0615 & 90.1408 & 9.8592 & 0.70504 \\
\hline 8 & 0.090575 & 0.42683 & 0.90558 & 85.5314 & 0.44154 & 89.5006 & 89.5006 & 10.4994 & 0.76452 \\
\hline 9 & 0.1246 & 0.49123 & 0.90055 & 87.0679 & 0.41893 & 92.4456 & 92.7017 & 7.2983 & 0.74254 \\
\hline 10 & 0.13671 & 0.50725 & 0.91152 & 87.58 & 0.41216 & 90.653 & 91.1652 & 8.8348 & 0.77572 \\
\hline 11 & 0.11897 & 0.47692 & 0.9162 & 87.9641 & 0.41453 & 91.9334 & 91.6773 & 8.3227 & 0.75346 \\
\hline 12 & 0.062242 & 0.35714 & 0.92552 & 88.4763 & 0.37513 & 91.6773 & 92.8297 & 7.1703 & 0.73315 \\
\hline Avg & 0.109105 & 0.458242 & 0.910366 & & & & & & 0.751576 \\
\hline
\end{tabular}


Table C-13: Complete training, test and verification set results for 15 variable non-linear test

\begin{tabular}{|r|r|r|r|r|r|r|r|r|r}
\hline \multicolumn{1}{|r|}{$\begin{array}{l}\text { Set } \\
\text { Sensitivity } \\
\text { Index }\end{array}$} & Sensitivity & Specificity & CR & ASE & bayes MDC & AprioriNeg & AprioriPos & ROC \\
\hline 1 & 0.17352 & 0.54468 & 0.9543 & 87.2449 & 0.41397 & 82.8231 & 80.017 & 19.983 & 0.84725 \\
\hline 2 & 0.1051 & 0.45161 & 0.91853 & 86.6184 & 0.44419 & 85.1718 & 88.7884 & 11.2116 & 0.75757 \\
\hline 3 & 0.11394 & 0.47222 & 0.90268 & 86.2996 & 0.42226 & 90.781 & 90.781 & 9.219 & 0.77085 \\
\hline 4 & 0.11904 & 0.47945 & 0.90819 & 86.8118 & 0.4174 & 90.3969 & 90.653 & 9.347 & 0.77052 \\
\hline 5 & 0.12973 & 0.49206 & 0.92479 & 88.9885 & 0.37641 & 92.7017 & 91.9334 & 8.0666 & 0.76689 \\
\hline 6 & 0.1138 & 0.46875 & 0.91353 & 87.7081 & 0.38523 & 90.9091 & 91.8054 & 8.1946 & 0.77204 \\
\hline 7 & 0.085298 & 0.41558 & 0.90625 & 85.7875 & 0.45294 & 92.0615 & 90.1408 & 9.8592 & 0.73521 \\
\hline 8 & 0.055385 & 0.34146 & 0.91273 & 85.2753 & 0.44497 & 89.5006 & 89.5006 & 10.4994 & 0.74395 \\
\hline 9 & 0.073238 & 0.38596 & 0.91713 & 87.8361 & 0.3979 & 92.4456 & 92.7017 & 7.2983 & 0.73603 \\
\hline 10 & 0.12092 & 0.47826 & 0.92275 & 88.3483 & 0.40606 & 90.525 & 91.1652 & 8.8348 & 0.7946 \\
\hline 11 & 0.099597 & 0.44615 & 0.90084 & 86.2996 & 0.41992 & 91.6773 & 91.6773 & 8.3227 & 0.77544 \\
\hline 12 & 0.037975 & 0.28571 & 0.92276 & 87.7081 & 0.40609 & 91.6773 & 92.8297 & 7.1703 & 0.63709 \\
\hline Avg & 0.094892 & 0.42656 & 0.913165 & & & & & & 0.750262 \\
\hline
\end{tabular}

Table C-14: Complete training, test and verification set results for 14 variable non-linear test

\begin{tabular}{|c|c|c|c|c|c|c|c|c|c|}
\hline Set & $\begin{array}{l}\text { Log } \\
\text { Sensitivity } \\
\text { Index }\end{array}$ & Sensitivity & Specificity & CR & ASE & bayes MDC & AprioriNeg & AprioriPos & ROC \\
\hline 1 & 0.06228 & 0.35745 & 0.92455 & 81.1224 & 0.54539 & 82.9082 & 80.017 & 19.983 & 0.77063 \\
\hline 2 & 0.12241 & 0.48387 & 0.91242 & 86.4376 & 0.43383 & 84.2676 & 88.7884 & 11.2116 & 0.77948 \\
\hline 3 & 0.090351 & 0.43056 & 0.88999 & 84.7631 & 0.44921 & 91.8054 & 90.781 & 9.219 & 0.76032 \\
\hline 4 & 0.11169 & 0.46575 & 0.91102 & 86.9398 & 0.43009 & 90.1408 & 90.653 & 9.347 & 0.74647 \\
\hline 5 & 0.11065 & 0.46032 & 0.9234 & 88.6044 & 0.3854 & 93.0858 & 91.9334 & 8.0666 & 0.76857 \\
\hline 6 & 0.087962 & 0.42188 & 0.90377 & 86.4277 & 0.40442 & 91.1652 & 91.8054 & 8.1946 & 0.7443 \\
\hline 7 & 0.052961 & 0.33766 & 0.89773 & 84.251 & 0.45444 & 91.9334 & 90.1408 & 9.8592 & 0.71751 \\
\hline 8 & 0.068538 & 0.37805 & 0.90272 & 84.7631 & 0.46925 & 90.0128 & 89.5006 & 10.4994 & 0.73325 \\
\hline 9 & 0.072273 & 0.38596 & 0.90746 & 86.9398 & 0.40942 & 92.5736 & 92.7017 & 7.2983 & 0.75183 \\
\hline 10 & 0.074818 & 0.3913 & 0.91011 & 86.4277 & 0.39461 & 91.1652 & 91.1652 & 8.8348 & 0.78069 \\
\hline 11 & 0.099597 & 0.44615 & 0.90084 & 86.2996 & 0.42373 & 91.9334 & 91.6773 & 8.3227 & 0.75982 \\
\hline 12 & 0.055436 & 0.33929 & 0.92414 & 88.2202 & 0.41261 & 92.3175 & 92.8297 & 7.1703 & 0.65383 \\
\hline Avg & 0.082428 & 0.405692 & 0.907118 & & & & & & 0.741659 \\
\hline
\end{tabular}


Table C-15: Complete training, test and verification set results for 13 variable non-linear test

\begin{tabular}{|r|r|r|r|r|r|r|r|r|r}
\hline \multicolumn{1}{r|}{$\begin{array}{l}\text { Set } \\
\text { Seg }\end{array}$} & $\begin{array}{l}\text { Sensitivity } \\
\text { Index }\end{array}$ & Sensitivity & Specificity & CR & ASE & bayes_MDC & AprioriNeg & AprioriPos & ROC \\
\hline 1 & 0.078063 & 0.39574 & 0.92242 & 81.7177 & 0.52812 & 82.398 & 80.017 & 19.983 & 0.78283 \\
\hline 2 & 0.10417 & 0.45161 & 0.91242 & 86.0759 & 0.42479 & 85.8951 & 88.7884 & 11.2116 & 0.8022 \\
\hline 3 & 0.082517 & 0.40278 & 0.93371 & 88.4763 & 0.38081 & 91.9334 & 90.781 & 9.219 & 0.78647 \\
\hline 4 & 0.098619 & 0.43836 & 0.92232 & 87.7081 & 0.3994 & 89.6287 & 90.653 & 9.347 & 0.77165 \\
\hline 5 & 0.065798 & 0.36508 & 0.93036 & 88.4763 & 0.35069 & 93.0858 & 91.9334 & 8.0666 & 0.78431 \\
\hline 6 & 0.064101 & 0.35938 & 0.93724 & 88.9885 & 0.3645 & 91.1652 & 91.8054 & 8.1946 & 0.74902 \\
\hline 7 & 0.065467 & 0.36364 & 0.93324 & 87.7081 & 0.40013 & 91.8054 & 90.1408 & 9.8592 & 0.74312 \\
\hline 8 & 0.06607 & 0.36585 & 0.9299 & 87.0679 & 0.42225 & 90.0128 & 89.5006 & 10.4994 & 0.76714 \\
\hline 9 & 0.060265 & 0.35088 & 0.93094 & 88.8604 & 0.36506 & 92.8297 & 92.7017 & 7.2983 & 0.76152 \\
\hline 10 & 0.10786 & 0.44928 & 0.94522 & 90.1408 & 0.33712 & 91.1652 & 91.1652 & 8.8348 & 0.80799 \\
\hline 11 & 0.073265 & 0.38462 & 0.92318 & 87.8361 & 0.37391 & 92.0615 & 91.6773 & 8.3227 & 0.76675 \\
\hline 12 & 0.044101 & 0.30357 & 0.93655 & 89.1165 & 0.37321 & 92.7017 & 92.8297 & 7.1703 & 0.70324 \\
\hline Avg & 0.072806 & 0.378344 & 0.932266 & & & & & & 0.764121 \\
\hline
\end{tabular}




\section{Appendix D - Best Performing Structure Results}

Table D-1: Initial ANN RFW parameter values for SNAPPE-II set and Ennett's model set (tables 31 and 33)

\begin{tabular}{ll}
\hline Parameter & ANN weights \\
\hline Error ratio & 1.02 \\
Lambda & 0.005 \\
Lambda decrement & 0.999 \\
Lambda increment & 1.001 \\
Learning rate & 0.0005 \\
Learning rate decrement & 0.8118 \\
Learning rate increment & 1.01 \\
Momentum & 0.99 \\
Weight scale factor & 0.01 \\
Float value & 0 \\
\hline
\end{tabular}

Figure D-1: Three-layered network with two hidden nodes and SNAPPE-II variable set using day 3 data and predicting mortality

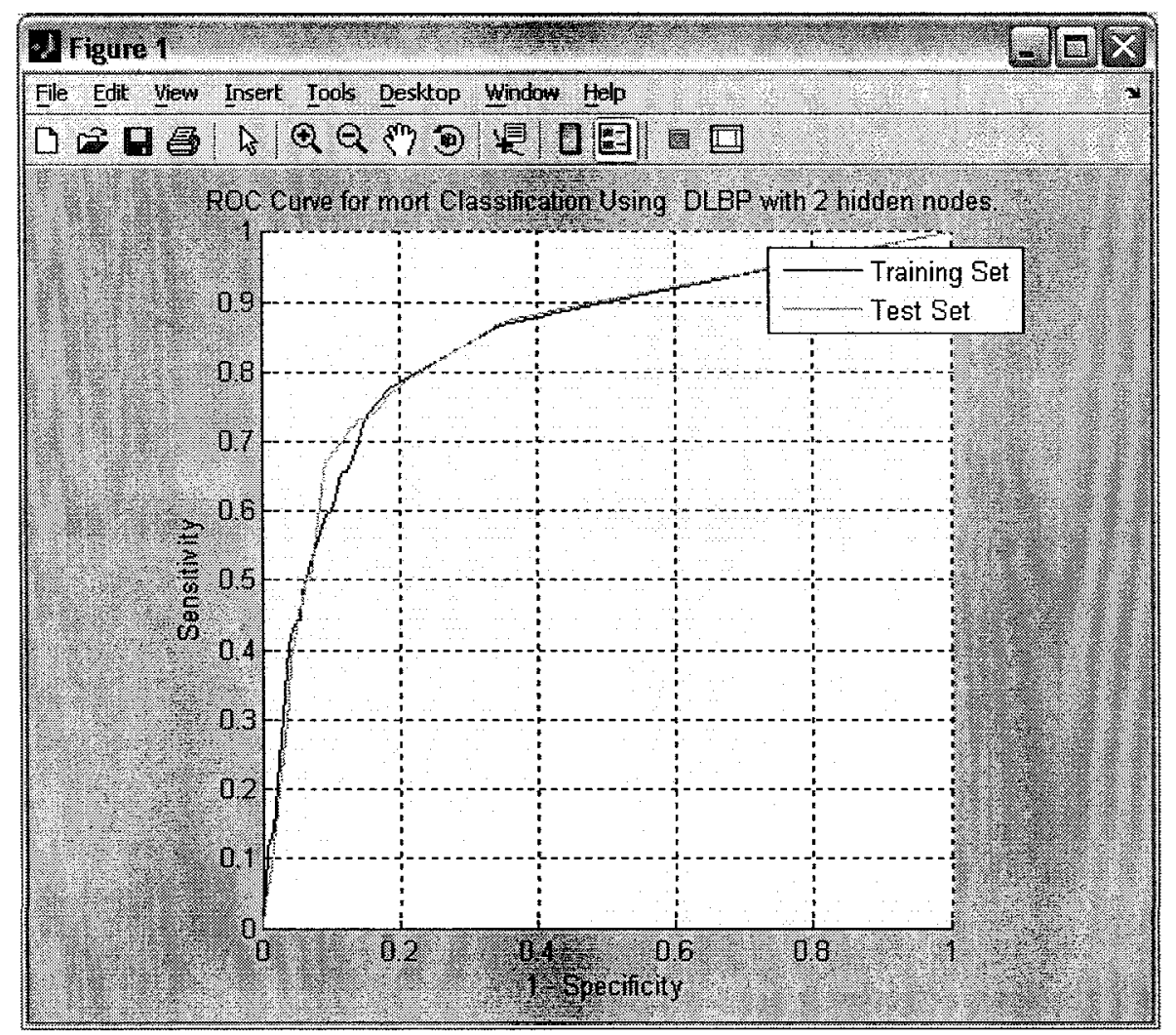


Table D-2: Complete training, test and verification set results for two hidden nodes and SNAPPE-II variable set using day 3 data and predicting mortality

\begin{tabular}{|r|r|r|r|r|r|r|r|r|r}
\hline \multicolumn{1}{|r|}{$\begin{array}{l}\text { Set } \\
\text { Seg }\end{array}$} & $\begin{array}{l}\text { Sensitivity } \\
\text { Index }\end{array}$ & Sensitivity & Specificity & CR & ASE & bayes MDC & AprioriNeg & AprioriPos & ROC \\
\hline 1 & 0.24167 & 0.66478 & 0.85296 & 82.4745 & 0.49544 & 88.1937 & 85.0085 & 14.9915 & 0.81649 \\
\hline 2 & 0.305 & 0.72727 & 0.85149 & 84.7932 & 0.37136 & 95.311 & 97.1345 & 2.8655 & 0.84137 \\
\hline 3 & 0.18774 & 0.60526 & 0.84332 & 83.7489 & 0.38985 & 97.2055 & 97.5494 & 2.4506 & 0.7992 \\
\hline 4 & 0.16614 & 0.57522 & 0.84446 & 83.7919 & 0.39503 & 97.313 & 97.5709 & 2.4291 & 0.79861 \\
\hline 5 & 0.24835 & 0.67376 & 0.84904 & 84.3723 & 0.38459 & 96.8401 & 96.969 & 3.031 & 0.81621 \\
\hline 6 & 0.12346 & 0.50407 & 0.85516 & 84.5873 & 0.38165 & 96.5391 & 97.356 & 2.644 & 0.77618 \\
\hline 7 & 0.17831 & 0.5873 & 0.85639 & 84.9097 & 0.36625 & 97.3775 & 97.2915 & 2.7085 & 0.80096 \\
\hline 8 & 0.081042 & 0.42276 & 0.84412 & 83.2975 & 0.40118 & 96.7326 & 97.356 & 2.644 & 0.75209 \\
\hline 9 & 0.1707 & 0.58 & 0.84864 & 84.2863 & 0.38819 & 97.5064 & 97.8504 & 2.1496 & 0.78631 \\
\hline 10 & 0.14798 & 0.54676 & 0.84822 & 83.9209 & 0.393 & 96.8186 & 97.012 & 2.988 & 0.78092 \\
\hline 11 & 0.12882 & 0.51613 & 0.84695 & 83.8134 & 0.39699 & 96.8616 & 97.3345 & 2.6655 & 0.76886 \\
\hline 12 & 0.16747 & 0.57944 & 0.83872 & 83.276 & 0.40097 & 97.1195 & 97.6999 & 2.3001 & 0.77873 \\
\hline Avg & 0.160001 & 0.55907 & 0.847502 & & & & & & 0.785807 \\
\hline
\end{tabular}


Figure D-2: Two-layered network (0 hidden nodes) result for Ennett's model dataset using day 3 data and predicting mortality

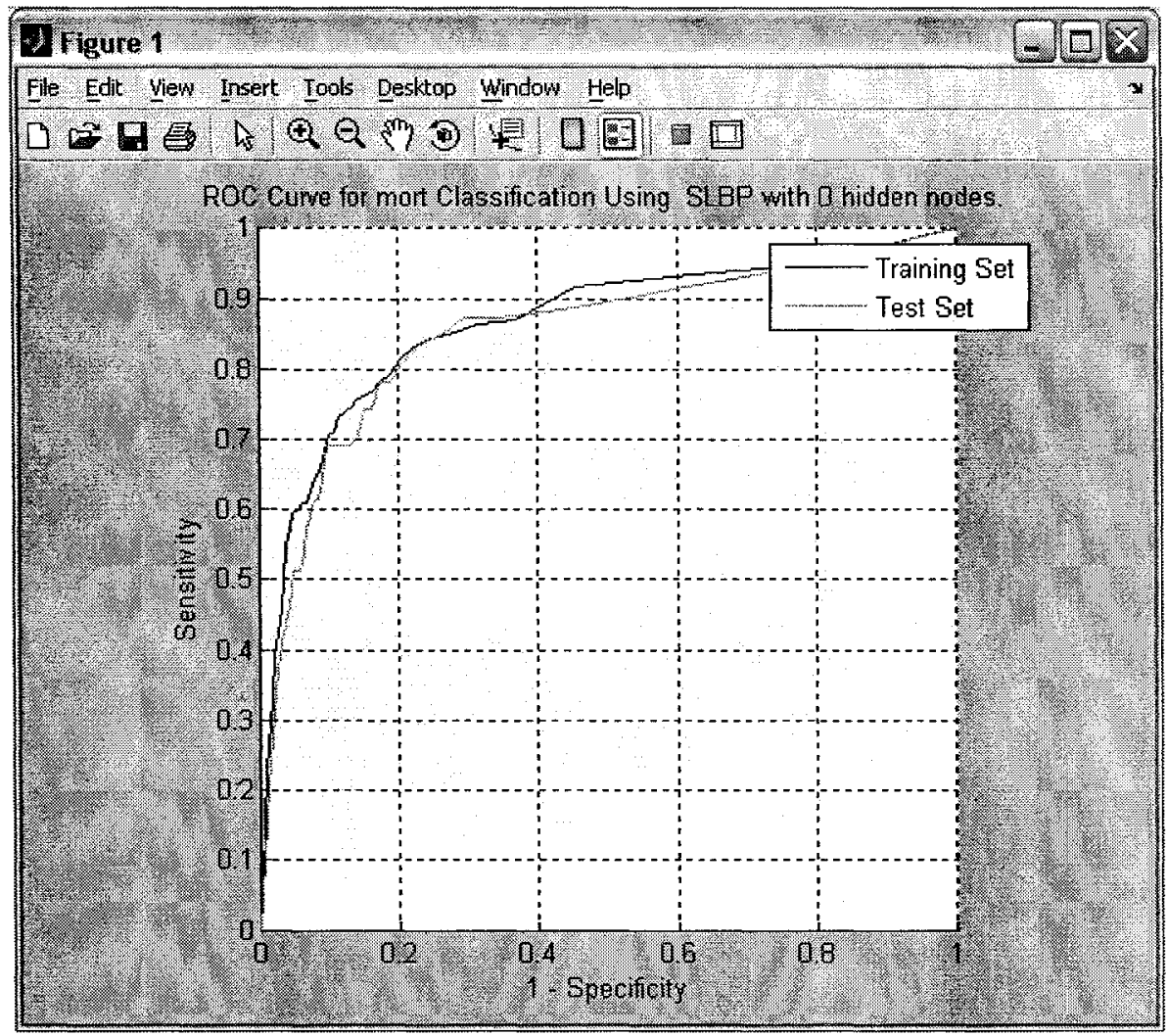

Table D-3: Complete training, test and verification set results for zero hidden nodes and Ennett variable set using day 3 data and predicting mortality

\begin{tabular}{|r|r|r|r|r|r|r|r|r|r}
\hline \multicolumn{1}{|l|}{ Set } & $\begin{array}{l}\text { Log } \\
\text { Sensitivity } \\
\text { Index }\end{array}$ & Sensitivity & Specificity & CR & ASE & bayes MDC & AprioriNeg & AprioriPos & ROC \\
\hline 1 & 0.31047 & 0.70647 & 0.90092 & 86.2023 & 0.42974 & 87.977 & 79.9973 & 20.0027 & 0.86567 \\
\hline 2 & 0.29583 & 0.69231 & 0.90444 & 89.9056 & 0.32865 & 93.227 & 97.4601 & 2.5399 & 0.85155 \\
\hline 3 & 0.32112 & 0.71212 & 0.90708 & 90.1548 & 0.32243 & 95.8298 & 97.1625 & 2.8375 & 0.86022 \\
\hline 4 & 0.24024 & 0.63793 & 0.90895 & 90.2193 & 0.33859 & 96.1952 & 97.5064 & 2.4936 & 0.80696 \\
\hline 5 & 0.22605 & 0.62595 & 0.90201 & 89.4239 & 0.34785 & 96.1737 & 97.184 & 2.816 & 0.82796 \\
\hline 6 & 0.28461 & 0.6789 & 0.91195 & 90.6492 & 0.32153 & 96.3027 & 97.6569 & 2.3431 & 0.86047 \\
\hline 7 & 0.36775 & 0.75 & 0.9022 & 89.8538 & 0.32615 & 96.7111 & 97.5924 & 2.4076 & 0.88628 \\
\hline 8 & 0.27732 & 0.67647 & 0.90308 & 89.8108 & 0.33764 & 96.2382 & 97.8074 & 2.1926 & 0.84555 \\
\hline 9 & 0.34997 & 0.73529 & 0.90545 & 90.0473 & 0.33091 & 96.0877 & 97.0765 & 2.9235 & 0.84992 \\
\hline 10 & 0.26096 & 0.66 & 0.90554 & 90.0258 & 0.3384 & 96.3242 & 97.8504 & 2.1496 & 0.82603 \\
\hline 11 & 0.21202 & 0.61207 & 0.89793 & 89.08 & 0.35819 & 96.5176 & 97.5064 & 2.4936 & 0.78351 \\
\hline 12 & 0.2971 & 0.69355 & 0.90415 & 89.8538 & 0.33717 & 96.1737 & 97.3345 & 2.6655 & 0.86217 \\
\hline Avg & 0.283714 & 0.678228 & 0.904834 & & & & & & 0.840907 \\
\hline
\end{tabular}


Table D-4: Initial ANN RFW parameter values for 13 variable linear model for day 3 data

\begin{tabular}{ll}
\hline Parameter & ANN weights \\
\hline Weight-elimination & Yes \\
Error ratio & 1.02 \\
Lambda & 0.005 \\
Lambda decrement & 0.999 \\
Lambda increment & 1.001 \\
Learning rate & 0.0005 \\
Learning rate decrement & 0.8118 \\
Learning rate increment & 1.001 \\
Momentum & 0.99 \\
Weight scale factor & 0.01 \\
Float value & 0 \\
\hline
\end{tabular}


Figure D-3: Three-layered structure with four hidden nodes using 13 variable linear model and day 3 data to predict mortality

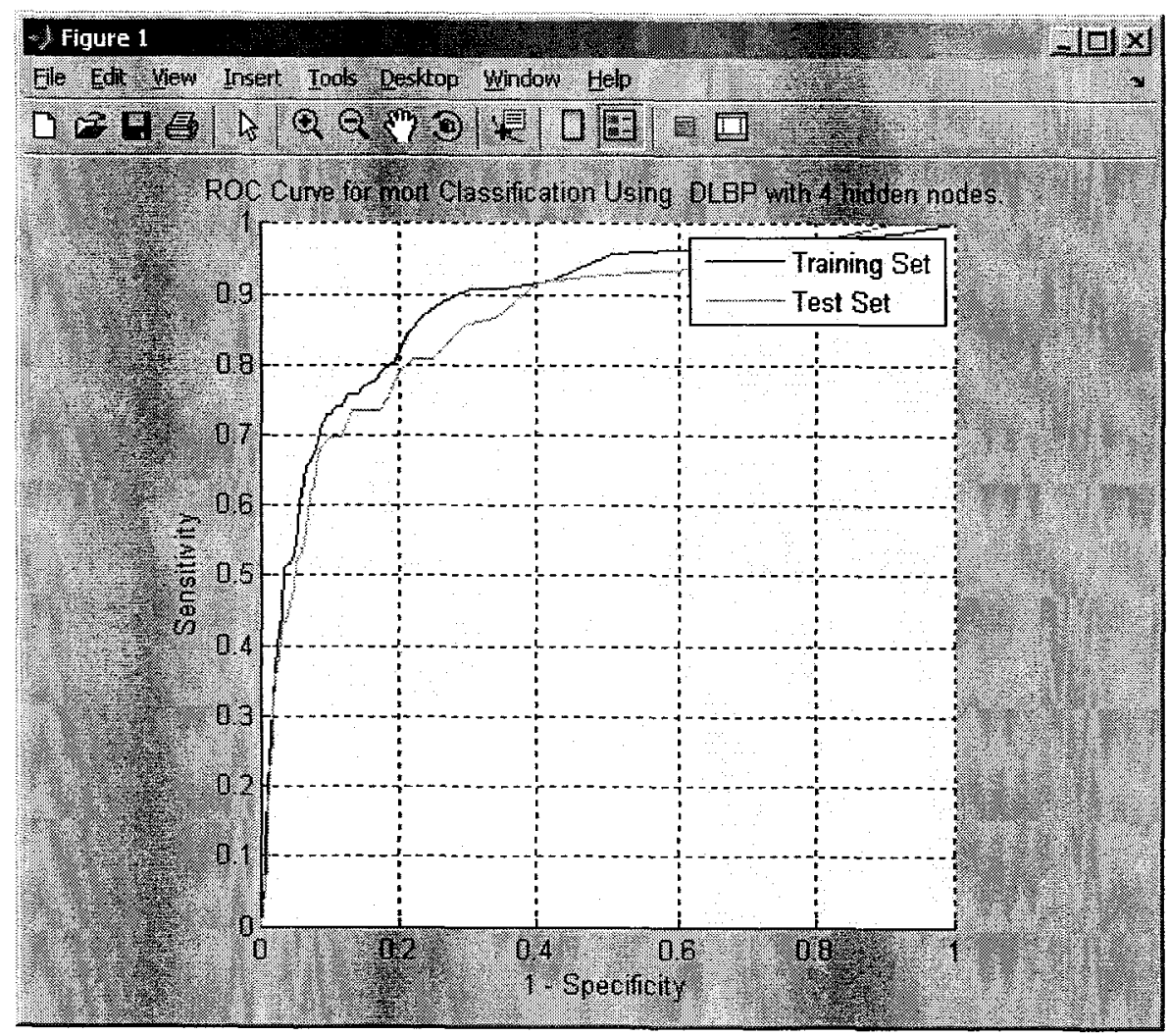

Table D-5: Complete training, test and verification set results for four hidden nodes and 13 variable linear model using day 3 data and predicting mortality

\begin{tabular}{|r|r|r|r|r|r|r|r|r|r}
\hline \multicolumn{1}{|l|}{ Set } & $\begin{array}{l}\text { Log } \\
\text { Sensitivity } \\
\text { Index }\end{array}$ & Sensitivity & Specificity & CR & ASE & bayes_MDC & AprioriNeg & AprioriPos & ROC \\
\hline 1 & 0.33102 & 0.71629 & 0.91439 & 87.4766 & 0.38809 & 86.4085 & 80 & 20 & 0.88935 \\
\hline 2 & 0.28374 & 0.6747 & 0.91934 & 91.2732 & 0.27 & 93.8131 & 97.2973 & 2.7027 & 0.86535 \\
\hline 3 & 0.28603 & 0.68 & 0.9123 & 90.6062 & 0.27609 & 96.5391 & 97.313 & 2.687 & 0.89192 \\
\hline 4 & 0.3196 & 0.70874 & 0.91141 & 90.6922 & 0.27059 & 96.7971 & 97.7859 & 2.2141 & 0.9089 \\
\hline 5 & 0.4081 & 0.77 & 0.91542 & 91.2296 & 0.26856 & 97.0765 & 97.8504 & 2.1496 & 0.90189 \\
\hline 6 & 0.29532 & 0.68548 & 0.91784 & 91.1651 & 0.27658 & 96.8616 & 97.3345 & 2.6655 & 0.87848 \\
\hline 7 & 0.39324 & 0.76 & 0.91606 & 91.1866 & 0.2628 & 96.7756 & 97.313 & 2.687 & 0.91336 \\
\hline 8 & 0.29514 & 0.68504 & 0.91845 & 91.2081 & 0.25894 & 96.8401 & 97.27 & 2.73 & 0.9056 \\
\hline 9 & 0.26804 & 0.66087 & 0.91845 & 91.2081 & 0.27672 & 96.1952 & 97.5279 & 2.4721 & 0.87534 \\
\hline 10 & 0.3193 & 0.70755 & 0.91333 & 90.8641 & 0.27169 & 96.3027 & 97.7214 & 2.2786 & 0.91017 \\
\hline 11 & 0.37747 & 0.74766 & 0.91925 & 91.5305 & 0.26698 & 96.5821 & 97.6999 & 2.3001 & 0.90704 \\
\hline 12 & 0.24718 & 0.64394 & 0.91128 & 90.3697 & 0.28744 & 96.1522 & 97.1625 & 2.8375 & 0.88377 \\
\hline & 0.320942 & 0.704928 & 0.915379 & & & & & & 0.897647 \\
\hline
\end{tabular}


Table D-6: Initial ANN RFW parameter values for 13 variable non-linear model for day 3 data

\begin{tabular}{ll}
\hline Parameter & ANN weights \\
\hline Weight-elimination & Yes \\
Error ratio & 1.005 \\
Lambda & $5 \mathrm{e}-005$ \\
Lambda decrement & 0.999 \\
Lambda increment & 1.001 \\
Learning rate & 0.0008 \\
Learning rate decrement & 0.9032 \\
Learning rate increment & 1.004 \\
Momentum & 0.99 \\
Weight scale factor & 0.01 \\
Float value & 0 \\
\hline
\end{tabular}

Figure D-4: Three-layered structure with four hidden nodes using 13 variable non-linear model and day 3 data to predict mortality

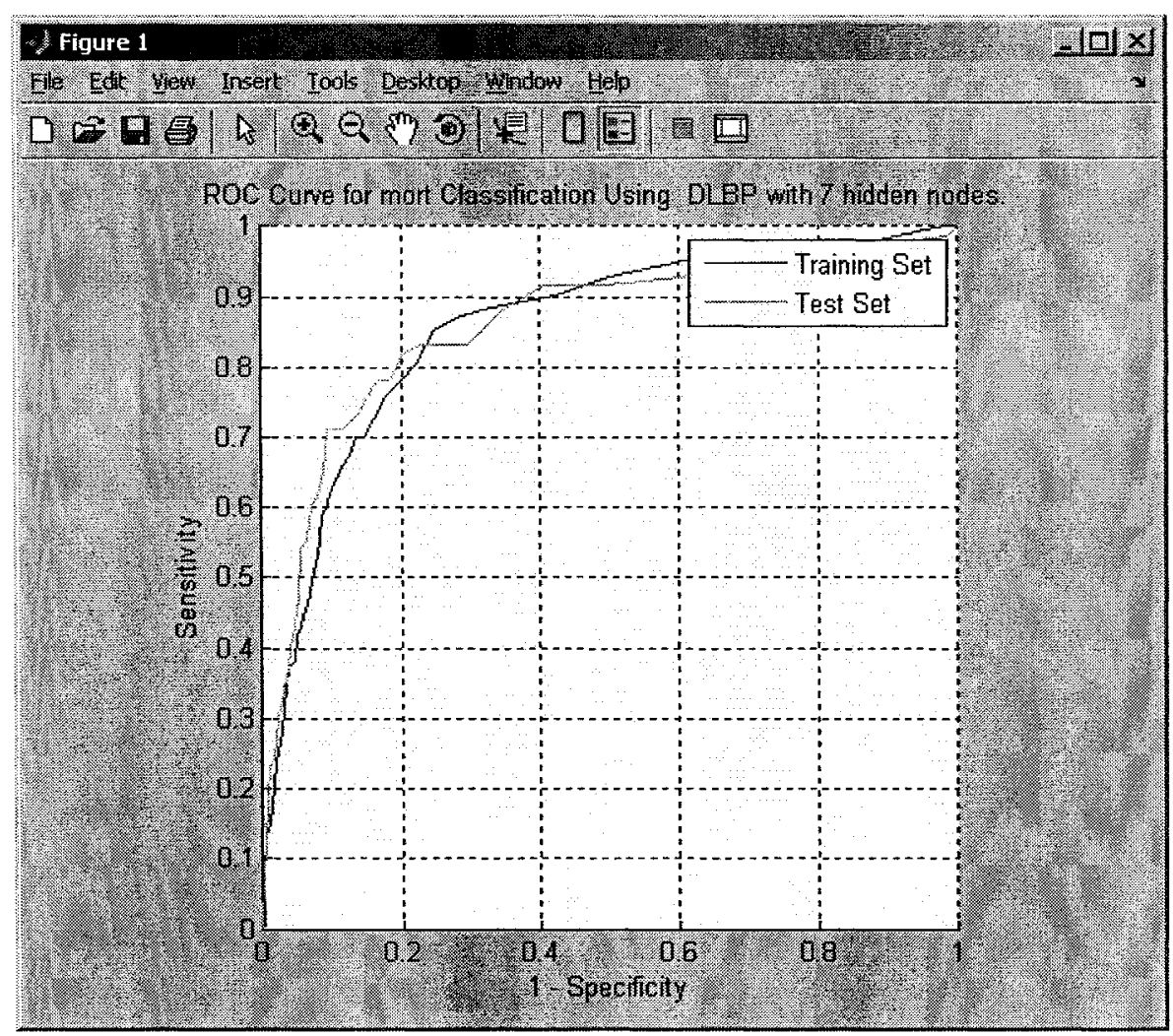


Table D-7: Complete training, test and verification set results for four hidden nodes and 13 variable linear model using day 3 data and predicting mortality

\begin{tabular}{|c|c|c|c|c|c|c|c|c|c|}
\hline Set & $\begin{array}{l}\text { Log } \\
\text { Sensitivity } \\
\text { Index }\end{array}$ & Sensitivity & Specificity & CR & ASE & bayes MDC & AprioriNeg & AprioriPos & ROC \\
\hline 1 & 0.22838 & 0.62817 & 0.9027 & 84.7797 & 0.4604 & 85.5808 & 80 & 20 & 0.85705 \\
\hline 2 & 0.3205 & 0.71084 & 0.90863 & 90.3289 & 0.30909 & 93.6177 & 97.2973 & 2.7027 & 0.86272 \\
\hline 3 & 0.25523 & 0.65254 & 0.90979 & 90.3267 & 0.31246 & 97.27 & 97.4635 & 2.5365 & 0.88225 \\
\hline 4 & 0.18197 & 0.57018 & 0.91274 & 90.4342 & 0.30769 & 97.098 & 97.5494 & 2.4506 & 0.86047 \\
\hline 5 & 0.22991 & 0.63 & 0.90224 & 89.6389 & 0.3244 & 97.184 & 97.8504 & 2.1496 & 0.87605 \\
\hline 6 & 0.28706 & 0.68103 & 0.91204 & 90.6277 & 0.30488 & 97.141 & 97.5064 & 2.4936 & 0.89582 \\
\hline 7 & 0.22212 & 0.61983 & 0.90642 & 89.8968 & 0.32172 & 96.6466 & 97.399 & 2.601 & 0.8748 \\
\hline 8 & 0.27019 & 0.66923 & 0.90447 & 89.7893 & 0.32723 & 96.4961 & 97.2055 & 2.7945 & 0.86574 \\
\hline 9 & 0.2842 & 0.67961 & 0.90965 & 90.4557 & 0.31063 & 97.0765 & 97.7859 & 2.2141 & 0.87953 \\
\hline 10 & 0.27727 & 0.67308 & 0.91029 & 90.4987 & 0.30248 & 96.7971 & 97.7644 & 2.2356 & 0.91396 \\
\hline 11 & 0.29379 & 0.6875 & 0.91079 & 90.5417 & 0.30445 & 97.27 & 97.5924 & 2.4076 & 0.89141 \\
\hline 12 & 0.23528 & 0.63636 & 0.90066 & 89.3164 & 0.3363 & 95.9802 & 97.1625 & 2.8375 & 0.87203 \\
\hline Avg & 0.253702 & 0.649936 & 0.907909 & & & & & & 0.881206 \\
\hline
\end{tabular}

Table D-8: ANN RFW parameters of best test run for best performing structure for each model

\begin{tabular}{lllll}
\hline Parameter & $\begin{array}{l}\text { SNAPPE-II } \\
\text { model }\end{array}$ & $\begin{array}{l}\text { Ennett's } \\
\text { model }\end{array}$ & $\begin{array}{l}\text { Linear } \\
\text { model }\end{array}$ & $\begin{array}{l}\text { Non-linear } \\
\text { model }\end{array}$ \\
\hline Best test epoch & 339 & 787 & 307 & 215 \\
Error ratio & 1.0518 & 1.02 & 1.02 & 1.005 \\
Lambda & 0.005 & 0.0001 & 0.005 & $5.5 \mathrm{e}-005$ \\
Lambda decrement & 0.999 & 0.999 & 0.999 & 0.999 \\
Lambda increment & 1.001 & 1.001 & 1.001 & 1.001 \\
Learning rate & 0.0003513 & 0.0004413 & 0.000361 & $5 \mathrm{e}-005$ \\
Learning rate decrement & 0.8118 & 0.8118 & 0.8118 & 0.9032 \\
Learning rate increment & 1.1255 & 1.01 & 1.1758 & 1.004 \\
Momentum & 0.99 & 0.99 & 0.99 & 0.99 \\
Weight scale factor & 0.01 & 0.01 & 0.01 & 0.01 \\
\hline
\end{tabular}




\section{Appendix E - T-test Results}

Artificial imputation using linear weights:

where 'lbloodp' is the original value of variable lbloodp and 'lbloodp2' is the imputed value

Variable lbloodp

Paired Samples Statistics

\begin{tabular}{|c|c|c|c|c|c|}
\hline & & Mean & $N$ & Std. Deviation & $\begin{array}{l}\text { Std. Error } \\
\text { Mean }\end{array}$ \\
\hline \multirow{2}{*}{$\begin{array}{l}\text { Pair } \\
1\end{array}$} & Ibloodp2 & 37.5356 & 686 & 6.07087 & .23179 \\
\hline & Ibloodp & 37.1778 & 686 & 8.85720 & .33817 \\
\hline
\end{tabular}

Paired Samples Correlations

\begin{tabular}{|l|r|r|r|}
\hline & \multicolumn{1}{|c|}{$\mathrm{N}$} & Correlation & \multicolumn{1}{c|}{ Sig. } \\
\hline Pair 1 Ibloodp2 \& Ibloodp & 686 & .506 & .000 \\
\hline
\end{tabular}

Paired Samples Test

\begin{tabular}{|c|c|c|c|c|c|c|c|c|}
\hline & \multicolumn{5}{|c|}{ Paired Differences } & \multirow[b]{3}{*}{$t$} & \multirow[b]{3}{*}{ df } & \multirow[b]{3}{*}{ Sig. (2-tailed) } \\
\hline & \multirow[b]{2}{*}{ Mean } & \multirow[b]{2}{*}{ Std. Deviation } & \multirow{2}{*}{$\begin{array}{c}\text { Std. Error } \\
\text { Mean }\end{array}$} & \multicolumn{2}{|c|}{$\begin{array}{l}95 \% \text { Confidence } \\
\text { Interval of the } \\
\text { Difference }\end{array}$} & & & \\
\hline & & & & Lower & Upper & & & \\
\hline Pair 1 lbloodp2 - Ibloodp & .35778 & 7.80095 & .29784 & -.22702 & .94257 & 1.201 & 685 & .230 \\
\hline
\end{tabular}

Variable po2fio2r

Paired Samples Statistics

\begin{tabular}{|ll|c|r|r|c|}
\hline & & Mean & $\mathrm{N}$ & Std. Deviation & $\begin{array}{c}\text { Std. Error } \\
\text { Mean }\end{array}$ \\
\hline Pair & po2fio2r2 & 2.0633 & 1532 & .31190 & .00797 \\
1 & po2fio2r & 2.0538 & 1532 & .93850 & .02398 \\
\hline
\end{tabular}

Paired Samples Correlations

\begin{tabular}{|l|r|r|r|}
\hline & $\mathrm{N}$ & Correlation & \multicolumn{1}{c|}{ Sig. } \\
\hline Pair 1 po2fio2r2 \& po2fio2r & 1532 & -.024 & .341 \\
\hline
\end{tabular}

Paired Samples Test

\begin{tabular}{|c|c|c|c|c|c|c|c|c|}
\hline & \multicolumn{5}{|c|}{ Paired Differences } & \multirow[b]{3}{*}{$\mathrm{t}$} & \multirow[b]{3}{*}{ df } & \multirow[b]{3}{*}{ Sig. (2-tailed) } \\
\hline & \multirow[b]{2}{*}{ Mean } & \multirow[b]{2}{*}{ Std. Deviation } & \multirow{2}{*}{$\begin{array}{c}\text { Std. Error } \\
\text { Mean }\end{array}$} & \multicolumn{2}{|c|}{$\begin{array}{l}95 \% \text { Confidence } \\
\text { Interval of the } \\
\text { Difference }\end{array}$} & & & \\
\hline & & & & Lower & Upper & & & \\
\hline Pair 1 po2fio 2 r2 - po2fio $2 r$ & .00947 & .99616 & .02545 & -.04045 & .05939 & .372 & 1531 & .710 \\
\hline
\end{tabular}

Variable lurine 
Paired Samples Statistics

\begin{tabular}{|ll|c|r|r|r|}
\hline & Mean & $\mathrm{N}$ & Std. Deviation & $\begin{array}{c}\text { Std. Error } \\
\text { Mean }\end{array}$ \\
\hline Pair & lurine2 & 3.7451 & 1175 & .51672 & .01507 \\
1 & lurine & 3.7332 & 1175 & 1.64511 & .04799 \\
\hline
\end{tabular}

\section{Paired Samples Correlations}

\begin{tabular}{|l|l|r|r|}
\hline & $\mathrm{N}$ & Correlation & Sig. \\
\hline Pair 1 lurine2 \& lurine & 1175 & .023 & .424 \\
\hline
\end{tabular}

Paired Samples Test

\begin{tabular}{|c|c|c|c|c|c|c|c|c|}
\hline & \multicolumn{5}{|c|}{ Paired Differences } & \multirow[b]{3}{*}{$t$} & \multirow[b]{3}{*}{ df } & \multirow[b]{3}{*}{ Sig. (2-tailed) } \\
\hline & \multirow[b]{2}{*}{ Mean } & \multirow[b]{2}{*}{ Std. Deviation } & \multirow{2}{*}{$\begin{array}{c}\text { Std. Error } \\
\text { Mean }\end{array}$} & \multicolumn{2}{|c|}{$\begin{array}{c}95 \% \text { Confidence } \\
\text { Interval of the } \\
\text { Difference }\end{array}$} & & & \\
\hline & & & & Lower & Upper & & & \\
\hline Pair 1 lurine2 - lurine & .01193 & 1.71280 & .04997 & -.08611 & .10996 & .239 & 1174 & .811 \\
\hline
\end{tabular}

\section{$\underline{\text { Variable Iserum }}$}

Paired Samples Statistics

\begin{tabular}{|c|c|c|c|c|c|}
\hline & & Mean & $\mathbf{N}$ & Std. Deviation & $\begin{array}{l}\text { Std. Error } \\
\text { Mean }\end{array}$ \\
\hline \multirow{2}{*}{$\begin{array}{l}\text { Pair } \\
1\end{array}$} & Iserum2 & 7.3125 & 1227 & .04211 & .00120 \\
\hline & Iserum & 7.3131 & 1227 & .08095 & .00231 \\
\hline
\end{tabular}

\section{Paired Samples Correlations}

\begin{tabular}{|l|r|r|r|}
\hline & $\mathrm{N}$ & Correlation & \multicolumn{1}{c|}{ Sig. } \\
\hline Pair 1 Iserum2 \& Iserum & 1227 & .389 & .000 \\
\hline
\end{tabular}

Paired Samples Test

\begin{tabular}{|c|c|c|c|c|c|c|c|c|}
\hline & \multicolumn{5}{|c|}{ Paired Differences } & \multirow[b]{3}{*}{$\mathrm{t}$} & \multirow[b]{3}{*}{ df } & \multirow[b]{3}{*}{ Sig. (2-tailed) } \\
\hline & \multirow[b]{2}{*}{ Mean } & \multirow[b]{2}{*}{ Std. Deviation } & \multirow{2}{*}{$\begin{array}{l}\text { Std. Error } \\
\text { Mean }\end{array}$} & \multicolumn{2}{|c|}{$\begin{array}{c}95 \% \text { Confidence } \\
\text { Interval of the } \\
\text { Difference }\end{array}$} & & & \\
\hline & & & & Lower & Upper & & & \\
\hline Pair 1 Iserum2 - Iserum & -.00064 & .07534 & .00215 & -.00486 & .00358 & -.297 & 1226 & .766 \\
\hline
\end{tabular}


Artificial imputation using non-linear weights:

where 'lbloodp' is the original value of variable lbloodp and 'lbloodp2' is the imputed value

Variable lbloodp

Paired Samples Statistics

\begin{tabular}{|ll|l|r|r|c|}
\hline & & & & Std. Error \\
& & Mean & $\mathrm{N}$ & Std. Deviation & \multicolumn{1}{c|}{ Mean } \\
\hline Pair & lbloodp2 & 37.5553 & 686 & 6.09201 & .23259 \\
1 & Ibloodp & 37.1778 & 686 & 8.85720 & .33817 \\
\hline
\end{tabular}

\section{Paired Samples Correlations}

\begin{tabular}{|l|r|r|r|}
\hline & \multicolumn{1}{|c|}{$\mathrm{N}$} & Correlation & \multicolumn{1}{c|}{ Sig. } \\
\hline Pair 1 Ibloodp2 \& Ibloodp & 686 & .505 & .000 \\
\hline
\end{tabular}

Paired Samples Test

\begin{tabular}{|c|c|c|c|c|c|c|c|c|}
\hline & \multicolumn{5}{|c|}{ Paired Differences } & \multirow[b]{3}{*}{$\mathrm{t}$} & \multirow[b]{3}{*}{ df } & \multirow[b]{3}{*}{ Sig. (2-tailed) } \\
\hline & \multirow[b]{2}{*}{ Mean } & \multirow[b]{2}{*}{ Std. Deviation } & \multirow{2}{*}{$\begin{array}{c}\text { Std. Error } \\
\text { Mean }\end{array}$} & \multicolumn{2}{|c|}{$\begin{array}{c}95 \% \text { Confidence } \\
\text { Interval of the } \\
\text { Difference }\end{array}$} & & & \\
\hline & & & & Lower & Upper & & & \\
\hline Pair 1 Ibloodp2 - Ibloodp & .37748 & 7.81334 & .29831 & -.20824 & .96320 & 1.265 & 685 & .206 \\
\hline
\end{tabular}

Variable po2fio2r

Paired Samples Statistics

\begin{tabular}{|ll|c|r|r|c|}
\hline & & & & \multicolumn{1}{|c|}{$\begin{array}{c}\text { Std. Error } \\
\text { Mean }\end{array}$} \\
\hline Pair & po2fio2r2 & 2.0593 & 1532 & .31650 & .00809 \\
1 & po2fio2r & 2.0538 & 1532 & .93850 & .02398 \\
\hline
\end{tabular}

Paired Samples Correlations

\begin{tabular}{|l|r|r|r|}
\hline & $\mathbf{N}$ & Correlation & Sig. \\
\hline Pair 1 po2fio2r2 \& po2fio2r & 1532 & -.017 & .497 \\
\hline
\end{tabular}

Paired Samples Test

\begin{tabular}{|c|c|c|c|c|c|c|c|c|}
\hline & \multicolumn{5}{|c|}{ Paired Differences } & \multirow[b]{3}{*}{$t$} & \multirow[b]{3}{*}{ df } & \multirow[b]{3}{*}{ Sig. (2-tailed) } \\
\hline & \multirow[b]{2}{*}{ Mean } & \multirow[b]{2}{*}{ Std. Deviation } & \multirow{2}{*}{$\begin{array}{c}\text { Std. Error } \\
\text { Mean }\end{array}$} & \multicolumn{2}{|c|}{$\begin{array}{c}95 \% \text { Confidence } \\
\text { interval of the } \\
\text { Difference }\end{array}$} & & & \\
\hline & & & & Lower & Upper & & & \\
\hline Pair 1 po2fio2r2 - po2fio2r & .00548 & .99564 & .02544 & -.04441 & .05538 & .216 & 1531 & .829 \\
\hline
\end{tabular}

$\underline{\text { Variable lurine }}$ 
Paired Samples Statistics

\begin{tabular}{|ll|c|r|r|c|}
\hline & & & & Std. Error \\
& Mean & $\mathrm{N}$ & Std. Deviation & Mean \\
\hline Pair & lurine2 & 3.7421 & 1175 & .51321 & .01497 \\
1 & lurine & 3.7332 & 1175 & 1.64511 & .04799 \\
\hline
\end{tabular}

Paired Samples Correlations

\begin{tabular}{|l|l|r|r|}
\hline & $\mathrm{N}$ & Correlation & Sig. \\
\hline Pair 1 lurine2 \& lurine & 1175 & .031 & .288 \\
\hline
\end{tabular}

Paired Samples Test

\begin{tabular}{|c|c|c|c|c|c|c|c|c|}
\hline & \multicolumn{5}{|c|}{ Paired Differences } & \multirow[b]{3}{*}{$t$} & \multirow[b]{3}{*}{ df } & \multirow[b]{3}{*}{ Sig. (2-tailed) } \\
\hline & \multirow[b]{2}{*}{ Mean } & \multirow[b]{2}{*}{ Std. Deviation } & \multirow{2}{*}{$\begin{array}{c}\begin{array}{c}\text { Std. Error } \\
\text { Mean }\end{array} \\
\end{array}$} & \multicolumn{2}{|c|}{$\begin{array}{c}95 \% \text { Confidence } \\
\text { interval of the } \\
\text { Difference }\end{array}$} & & & \\
\hline & & & & Lower & Upper & & & \\
\hline Pair 1 lurine2 - lurine & .00897 & 1.70804 & .04983 & -.08879 & .10673 & .180 & 1174 & .857 \\
\hline
\end{tabular}

\section{Variable lserum}

Paired Samples Statistics

\begin{tabular}{|ll|c|r|r|c|}
\hline & Mean & $\mathrm{N}$ & Std. Deviation & $\begin{array}{c}\text { Std. Error } \\
\text { Mean }\end{array}$ \\
\hline Pair & Iserum2 & 7.3114 & 1227 & .04260 & .00122 \\
1 & Iserum & 7.3131 & 1227 & .08095 & .00231 \\
\hline
\end{tabular}

Paired Samples Correlations

\begin{tabular}{|l|l|r|r|}
\hline & $\mathrm{N}$ & Correlation & \multicolumn{1}{c|}{ Sig. } \\
\hline Pair 1 Iserum2 \& Iserum & 1227 & .409 & .000 \\
\hline
\end{tabular}

Paired Samples Test

\begin{tabular}{|c|c|c|c|c|c|c|c|c|}
\hline & \multicolumn{5}{|c|}{ Paired Differences } & \multirow[b]{3}{*}{$\mathrm{t}$} & \multirow[b]{3}{*}{ df } & \multirow[b]{3}{*}{ Sig. (2-tailed) } \\
\hline & \multirow[b]{2}{*}{ Mean } & \multirow[b]{2}{*}{ Std. Deviation } & \multirow{2}{*}{$\begin{array}{l}\text { Std. Error } \\
\text { Mean }\end{array}$} & \multicolumn{2}{|c|}{$\begin{array}{c}95 \% \text { Confidence } \\
\text { Interval of the } \\
\text { Difference }\end{array}$} & & & \\
\hline & & & & Lower & Upper & & & \\
\hline Pair 1 Iserum2 - Iserum & -.00173 & .07448 & .00213 & -.00590 & .00244 & -.815 & 1226 & .415 \\
\hline
\end{tabular}

